\title{
CASSINI RADIO SCIENCE
}

\author{
A. J. KLIORE ${ }^{1, *}$, J. D. ANDERSON ${ }^{1}$, J. W. ARMSTRONG ${ }^{1}$, S. W. ASMAR ${ }^{1}$, \\ C. L. HAMILTON ${ }^{1}$, N. J. RAPPAPORT ${ }^{1}$, H. D. WAHLQUIST ${ }^{1}$, R. AMBROSINI ${ }^{2}$, \\ F. M. FLASAR ${ }^{3}$, R. G. FRENCH ${ }^{4}$, L. IESS ${ }^{5}$, E. A. MAROUF ${ }^{6}$ and A. F. NAGY ${ }^{7}$ \\ ${ }^{1}$ Jet Propulsion Laboratory, California Institute of Technology, 4800 Oak Grove Drive, \\ Pasadena, CA 91109, USA \\ ${ }^{2}$ Istituto di Radioastronomia CNR, Via Gobetti 101, I-40129, Bologna, Italy \\ ${ }^{3}$ NASA-Goddard Space Flight Center, Greenbelt, MD 20771, USA \\ ${ }^{4}$ Wellesley College, Wellesley, MA 02481, USA \\ ${ }^{5}$ Università di Roma 'La Sapienza', Via Eudossiana 18, I-00184 Roma, Italy \\ ${ }^{6}$ San Jose State University, One Washington Square, San Jose, CA 95192, USA \\ ${ }^{7}$ University of Michigan, 2455 Hayward Avenue, Ann Arbor, MI 48109, USA \\ (*author for correspondence, e-mail:akliore@jpl.nasa.gov)
}

(Received 29 December 1999; Accepted in final form 4 April 2002)

\begin{abstract}
Cassini radio science investigations will be conducted both during the cruise (gravitational wave and conjunction experiments) and the Saturnian tour of the mission (atmospheric and ionospheric occultations, ring occultations, determinations of masses and gravity fields). New technologies in the construction of the instrument, which consists of a portion on-board the spacecraft and another portion on the ground, including the use of the Ka-band signal in addition to that of the S- and X-bands, open opportunities for important discoveries in each of the above scientific areas, due to increased accuracy, resolution, sensitivity, and dynamic range.
\end{abstract}

Keywords: atmospheres, Cassini, gravitational fields, gravitational waves, general relativity, ionospheres, occultations, planetary rings, radio science, Saturn, Titan

\section{Introduction}

This paper, produced by the Cassini Radio Science Team during the early cruise of the Cassini spacecraft en route for the Saturnian system, records major reference information concerning the investigations to be conducted, and the instrument that will be operated to conduct these investigations. Section 2 describes the radio science investigations. Section 3 is devoted to the radio science instrument. Section 4 contains a brief conclusion.

\section{Radio Science Investigations}

For each of the radio science investigations:

- Gravitational wave experiments,

- Conjunction experiments (a new test of general relativity, study of the solar corona),

Space Science Reviews 115: 1-70, 2004.

(C) 2004 Kluwer Academic Publishers. Printed in the Netherlands. 
- Gravitational field measurements and celestial mechanics experiments (pertaining to Saturn, Titan, and the Icy Satellites),

- Ring occultation experiments,

- Atmospheric and ionospheric occultation experiments (pertaining to Saturn, Titan, and the icy satellites),

we describe the scientific objectives with respect to the present state of knowledge, the techniques, and the major requirements.

\subsection{GRAVitational WAVE EXPERIMENTS}

\subsubsection{Scientific Objectives}

This section of the paper outlines the method and experimental setup of the Cassini Gravitational Wave Experiment (GWE).

The extensive and stringent tests of gravitation theories carried out in the solar system, together with observations of binary pulsars, have dispelled most doubts about Einstein's theory of general relativity and the existence of the gravitational waves it predicts. The direct detection of gravitational waves constitutes an outstanding challenge for experimental physics, however, and - when successful will open up a new window for observational astronomy (Thorne, 1987). Since gravitational waves are virtually unaffected by intervening matter, their observation will probe the dynamics of cataclysmic events in the deep interiors of, for example, supernovae and active galactic nuclei, regions which are inaccessible to electromagnetic observations.

There are three main frequency bands of astronomical interest:

- The "high" frequency band, around $1 \mathrm{kHz}$, where the sources include supernovae and stellar coalescences;

- The "low" frequency band, around $1 \mathrm{mHz}$, where the sources include compact binary systems such as a binary pulsar, the formation of super-massive black holes, and the coalescence of supermassive binary black holes;

- The "very low" frequency band, $<1 \mu \mathrm{Hz}$, where one might expect a stochastic background of waves from the superposition of stellar binaries, distant past collapse events, and possibly the red-shifted remnant of a primordial cosmic background created by density fluctuations in the Big Bang. (See Armstrong et al., 2003 for results from Cassini's GWE).

The variety of possible sources in the low-frequency band accessible to spacecraft Doppler tracking necessitates using a variety of signal detection techniques. This is especially true because it is a search conducted largely "in the dark"; while astronomical observations and theory almost guarantee the existence of gravitational waves, it is still beyond our capability to predict the shape, or even the likely strength, of waves from these sources. So the best one can do is to do a systematic search for a variety of plausible waveforms (wide-band pulses, periodic and quasi-periodic waveforms, stochastic backgrounds). 
For detection purposes, an ideal source would be the binary pulsar $1913+16$. Binary pulsars are objects for which clear evidence of gravitational radiation already exists and everything one needs to know about the system is available to predict precisely the observed signal (Wahlquist, 1987). The wave frequency of the strongest harmonic of the periodic signal from $1913+16$ falls exactly where the Doppler method is most sensitive. Unfortunately, the predicted amplitude of the signal from $1913+16$ is too weak to detect at Cassini's sensitivity by many orders of magnitude.

In addition to being of great intrinsic astrophysical interest, supermassive binary black hole systems may be the most promising sources for detection with Cassini. Clean binary systems, not too close to coalescence, are sufficiently simple that detailed comparison of theory and observation is possible. Of course, since the parameters of the system are not known in advance, it is necessary to employ a large ensemble of signal templates to search for waves from one of these systems. Near coalescence, the amplitude and frequency of the wave increase with time; special methods to extract these "chirp" signals from both random and systematic noises have been developed (Tinto and Armstrong, 1991; Anderson et al., 1993; Bertotti et al., 1994; Bertotti, 1997; Iess and Armstrong, 1997; Bertotti et al., 1999). Very close to coalescence, physical processes become more complicated and templates may at best be a rough approximation to the emitted waveforms.

The existence of binary sources has become increasingly plausible with the recent observations of supermassive objects in galactic nuclei, together with evidence for the frequent merging of galaxies in the early universe when their spatial density was much higher than at present. Cassini should be able to detect signals of this type, if they are present in the Doppler frequency band with the expected strength, well beyond the Virgo cluster $(\approx 17 \mathrm{Mpc})$, thus including thousands of candidate galaxies.

The gravitational wave search on the Cassini mission has been the most sensitive Doppler experiment ever performed. The experiment has been repeated three times during the cruise period from Jupiter to Saturn; i.e., when the spacecraft was the antisolar direction from earth (November 2001-January 2002; December 2002-January 2003; December 2003-January 2004). At each opposition, Cassini has been Doppler-tracked as continuously as possible for 40 days. Around-theclock tracking required using all three deep space network (DSN) complexes (Goldstone, Madrid, Canberra), and may be supported additionally by non-DSN radio antennas in Italy. The highest sensitivity was achieved with DSS-25, a beam waveguide antenna located at the Goldstone complex, which has been carefully designed for the utmost in frequency stability and which has Ka-band uplink, precision frequency standards, and advanced tropospheric correction equipment.

Cassini at opposition became one of the largest gravitational wave antenna's ever used $(\approx 8 \mathrm{AU}$ in length), attaining by far the highest sensitivity to date for gravitational waves at the lower end of the low-frequency band. 


\subsubsection{Techniques}

Detection methods for gravitational radiation depend on the time scale of the radiation. At high frequencies the main techniques are resonant bars and laser interferometers. These techniques achieve excellent sensitivity in the Fourier frequency band where they operate. For frequencies lower than about $10 \mathrm{~Hz}$, however, it becomes prohibitively difficult to isolate these detectors from seismic noise, other acoustic noise in the environment, and from uctuating gravity gradients. For observations of sources radiating in the low frequency band (approximately $0.0001 \mathrm{~Hz}$, or lower, to $0.01 \mathrm{~Hz}$ ), the detector must be spaceborne. Although at the moment the low-frequency band is explored by means of Doppler tracking, the next generation of space-based detectors will soon become a reality with the launch of the USA mission, jointly funded by NASA and ESA (Bender et al., 1998). For very-low frequencies (below about $10^{-6} \mathrm{~Hz}$ ) pulsar timing can be used to search for stochastic gravitational waves.

In the spacecraft technique, the earth and a distant spacecraft act as separated test masses. The Doppler tracking system measures the relative dimensionless velocity of the earth with respect to the spacecraft

$$
2 \frac{\Delta v}{c}=\frac{\Delta v}{v_{0}}=y
$$

as a function of time; $\Delta v$ is the perturbation of the Doppler frequency from $\Delta v_{0}$, the nominal radio frequency. A gravitational wave of amplitude $h$ incident on the system causes small perturbations in the tracking record. These perturbations are of order $h$ in $y$ and are replicated three times in the Doppler data (Estabrook and Wahlquist, 1975). That is, the gravitational wave signal in the observed Doppler time series is the convolution of the waveform

$$
s(t)=\left(1-\mu^{2}\right)^{-1} \mathbf{n} \cdot\left[h_{+}(t) \mathbf{e}_{+}+h_{\times}(t) \mathbf{e}_{\times}\right] \cdot \mathbf{n}
$$

with the three-pulse response function

$$
r(t)=\frac{\mu-1}{2} \delta(t)-\mu \delta\left(t-(1+\mu) \frac{L}{c}\right)+\frac{1+\mu}{2} \delta\left(t-2 \frac{L}{c}\right)
$$

Here $\mu$ is the cosine of the angle between the earth-spacecraft vector and the gravity wavevector, $L$ is the earth-spacecraft distance, $\mathbf{n}$ is the unit vector from the earth to the spacecraft, $h_{+}(t)$ and $h_{\times}(t)$ are the gravity waveforms for each polarization and $\mathbf{e}_{+}$and $\mathbf{e}_{\times}$are transverse, traceless polarization tensors (Estabrook and Wahlquist, 1975; Wahlquist et al., 1977; Wahlquist, 1987). The sum of the three pulses is zero; hence burst waves having a duration longer than about $L / c$ overlap in the tracking record and the net response cancels to first order. The tracking system thus has a passband where it has maximum sensitivity: below about $c / L$, by pulse cancellation, the response is proportional to $f$; thermal 
noise in the radio system and the short-term stability of the frequency standard to which the Doppler system is referenced limits the high-frequency response to $f \approx 0.1 \mathrm{~Hz}$.

\subsubsection{Major Requirements}

The anticipated signal amplitudes depend on the source generating the wave and the earth-source distance, but are in any case expected to be small. Since the resulting perturbations in the Doppler record are also expected to be small, careful attention to noise and systematics is required.

Non-signal fluctuations in the Doppler time series are caused by charged particle scintillation, tropospheric scintillation, antenna mechanical noise, clock noise, spacecraft unmodeled motion, ground electronic noise, thermal noise in the radio link, spacecraft electronics noise, and systematic errors. The extent to which these noises affect the ultimate sensitivity, depends on the gravity waveform because the noises enter the observable with different transfer functions (e.g., Wahlquist et al., 1977; Estabrook, 1978; Armstrong, 1989; Bertotti et al., 1999). However, it is clearly important to minimize the absolute level of the noises. (A more complete discussion of the error budget for precision Doppler tracking, with particular attention to the Cassini gravitational wave experiment, is given in Armstrong (1998), Tinto and Armstrong (1998) and Asmar et al. (2004)).

Obviously any unmodeled motion of the spacecraft itself enters directly in the Doppler record. Thus the Cassini gravitational wave experiment requires that activity on the spacecraft causing unmodeled motions be minimized during gravitational wave observations.

Propagation noise ("scintillation") arises from irregularities in the refractive index along the radio path. These fluctuations randomly advance and retard the phase of the wave and thus cause frequency fluctuations. Charged particle scintillation, which is dominated by solar wind scintillation, can be minimized by observing at large sun-earth-spacecraft angles and by observing at high-radio frequency. The Cassini gravitational wave experiment thus requires that observations be made in the antisolar direction. The Cassini gravitational wave experiment has been the first scientific user of Ka-band $(\approx 32 \mathrm{GHz})$ up and downlinks. This has dramatically reduced the contribution of charged particle scintillation relative to previous experiment. Scintillation in the neutral atmosphere is an important noise source. Cassini gravitational wave experiments requires water-vapor radiometers colocated with DSS-25 (the DSN Ka-band uplink station) to estimate and remove the tropospheric scintillation to acceptable levels.

Clock noise is fluctuation in the frequency standard that drives the Doppler system. This noise is fundamental and must be minimized for a successful experiment. Cassini-era frequency standards have been engineered for excellent stability in the Fourier band of interest to the gravitational wave experiment and are expected to enter the observable at a noise level less than or comparable to the other principal noise sources. 
Antenna mechanical noise is physical motion of the antenna and feed system as the ground antenna moves to track the spacecraft, deforms under gravity, distorts due to wind, etc. This noise source has been negligible in previous gravitational wave experiments, but has been detected at the excellent sensitivity of the Cassini Ka-band system.

\subsection{CONJUNCTION EXPERIMENTS}

Radio science experiments near solar conjunctions have been exploited for tests of general relativity and probing of the solar corona. The unique radio system of Cassini allows singling out plasma effects on carrier phase and is therefore expecially suited for Doppler measurements near the sun. The 2002 Cassini solar conjunction experiment has yielded the most accurate test of general relativity so far.

\subsubsection{A New Test of General Relativity}

2.2.1.1. Scientific Objectives. The gravitational deflection of light rays, a crucial test of the theory of general relativity, has been performed so far with two methods:

- by measuring the differential deflection of the apparent position of stars or radio sources near the Sun;

- by measuring the change in the light transit time from a spacecraft near solar conjunction.

Within the parametrized post-Newtonian (PPN) (Will, 1993) approximation in its minimal form, in which the metric depends on two dimensionless parameters $\gamma$ and $\beta$, these effects are controlled by $\gamma$. Before the Cassini experiment this parameter was constrained to be within $10^{-3}$ of unity, the general relativistic value. Several experiments have been done in the past, all more or less with similar results, and it was apparent that new ideas and instrumentation were needed to obtain a major increase in accuracy. It is remarkable that the test performed more than 20 years ago by measuring the two-way travel time of radio signals from the earth to the Viking landers (Reasenberg et al., 1979; Borderies et al., 1980) has been marginally improved only very recently (Eubanks et al., 1997), using more accurate VLBI (very-long-baseline interferometry) techniques for precision deflection measurements.

In the past, these experiments have played an essential role in the rejection of several alternative theories of gravity and in strengthening our confidence in the theory of general relativity. The main candidate for an alternative theory within this constraint is a scalar field coupled to the metric, which is the most obvious way to produce an inflationary cosmology; as the Universe starts its decelerated expansion phase, this field becomes progressively weaker, but its remnant, still present today, determines a small correction to the parameters $\gamma$ and $\beta$. In the absence of a 
reliable theory of inflation, it is difficult to assess the order of magnitude of these corrections; however, values from $10^{-7}$ to $10^{-5}$ have been considered (Damour and Nordtvedt, 1993). It is therefore most important to devise new methods to improve the measurement of $\gamma$.

The new technological developments required by the Cassini mission, in particular, the use of Ka-band radio links have allowed us to test general relativity to a substantially greater accuracy. The outstanding radio system of the spacecraft and ground station allows a nearly complete calibration of the plasma noise and an unprecedented stability of the interplanetary link. A crude estimation of the expected accuracies (Iess et al., 1999) indicated that $\gamma$ could be measured to levels of about $10^{-5}$, almost two orders of magnitude better than the previous experimental accuracy. The analysis of the data aquired between June 6 and July 5, 2002 fully confirmed the expectations: the experimental value for $\gamma$ was found to be $1+(2.1$ $\pm 2.3) 10^{(-5)}$ (Bertotti et al., 2003).

2.2.1.2. Techniques. The measurement of $\gamma$ with Cassini is based upon a third, new observable: the frequency shift induced on a radio wave when the spacecraft is near solar conjunction (Bertotti and Giampieri, 1992; Iess et al., 1999). Solar gravity has an effect on the frequencies of photons, since the relative frequency shift $y=\Delta v / v$ due to the solar metric is just a time derivative of the delay of radio signals measured in space experiments:

$$
y=\frac{d}{d t} \Delta \tau,
$$

where

$$
\Delta \tau=(1+\gamma) M_{\odot} \ln \frac{r_{1}+r_{2}+r_{12}}{r_{1}+r_{2}-r_{12}},
$$

expressed in terms of the geometrical units in which $G=c=1$ and $M_{\odot}=5 \mu \mathrm{s}$, has the well-known dependence on the sun-earth, sun-spacecraft and earth-spacecraft distances $r_{1}, r_{2}$ and $r_{12}$. For a two-way link, as in the case of Cassini, $y$ is actually the sum of two contributions, respectively from the uplink and the downlink. Near conjunctions, Equation (5) can be approximated using the impact parameter $b$ of the beam:

$$
\Delta \tau \approx 2(1+\gamma) M_{\odot} \ln \frac{4 r_{1} r_{2}}{b^{2}} .
$$

The corresponding value of $y$ is therefore

$$
y \approx 4(1+\gamma) \frac{M_{\odot}}{b} \dot{b} .
$$

In the case of Cassini, with a nearly grazing conjunction, $y \approx 10^{-9}$, five orders of magnitude larger than the expected stability of the radio link at time scales of $10^{4}$ $\mathrm{s}\left(\sigma_{y}=3 \times 10^{-15}\right)$. This rough comparison indicated that the Cassini experiment could lead to a test of general relativity with unprecendented accuracy. 
Another instructive way of looking at this experiment is to consider the effect on the frequencies as a consequence of the solar gravitational deflection. For a beam with impact parameter $b$, the deflection angle

$$
\delta=2(1+\gamma) \frac{M_{\odot}}{b}
$$

changes in time, therefore producing a variation in the direction of arrival $\mathbf{n}$ of photons. Since the observable quantity is essentially the radial velocity $\mathbf{v} \cdot \mathbf{n}$ of the spacecraft with respect to the earth, a deflection by an angle $\delta$ changes $\mathbf{n}$ and therefore produces an additional velocity along the line of sight of order $v \delta$. Again, for a grazing incidence, this amounts to approximately $10^{-9}$.

The relativistic Doppler signal evolves over time scales of order $b /$ (orbital velocity) $\approx 10^{5} \mathrm{~s}$. Thus, a Doppler experiment requires short observation periods to minimize the effects of non-gravitational accelerations. On the other hand, large Doppler signals are obtained only at small impact parameters, when the radio beam, well inside the solar corona, undergoes strong frequency fluctuations. Until the recent implementation of multi-frequency radio links at X- and Ka-bands, which allows a full plasma calibration, Doppler experiments could not compete with the more familiar tests based on range measurements.

The main hindrance to precise measurements near conjunctions is indeed the solar corona, whose large and changing electron density induces severe propagation effects. In the past, spacecraft tracking near solar conjunction using S- and X-band radio links has provided important information about the coronal plasma. Since the coronal contribution to the fractional frequency change $y$ is inversely proportional to the square of the carrier frequency $v$, the noise due to the corona is particularly large at the lower frequencies, such as S-band. With Cassini, besides the standard communication link in X-band (7.1-8.4 GHz), an additional link in Ka-band (32$34 \mathrm{GHz}$ ) was available, with two neighbouring downlink carriers driven by the Ka-band uplink and the X-band uplink, respectively. This configuration, with multifrequency transmission from the ground and the spacecraft, will allow for the first time a complete plasma calibration both in the uplink and the downlink (Bertotti et al., 1993). Moreover, the use of higher frequency carriers makes the link nearly immune to frequency jitter and cycle slips. The experimental results showed an excellent phase stability (about $2 \times 10^{-14}$ or smaller), a value generally obtained close to solar opposition (Tortora et al., 2003; Tortora et al., 2004).

2.2.1.3. Major Requirements. The experiment was planned during two useful conjunctions in the cruise phase, in June 2002 and June 2003, at times when there was very little activity on the spacecraft. Unfortunately, due to a malfunction of the key onboard instrument (the $\mathrm{Ka} / \mathrm{Ka}$ frequency translator, see sect. 3.2), only the data collected in 2002 have been used. The very small inclination of the Cassini orbit makes the geometry of the experiment particularly favorable, with a minimum impact parameters of $1.6 R_{\odot}$. The main tracking station was DSS-25, the only DSN 
station capable of supporting a full multi-frequency link (with uplink both at Xand Ka-band).

Measuring $\gamma$ to levels of $10^{-5}$ requires a very stable radio link, with an accurate calibration for the effects of the media (solar corona and troposphere). The instrumental specifications set by the gravitational wave experiments are adequate, but now the complete multifrequency link becomes essential.

While the two experiments share a good degree of commonality (after all, they are based upon the same observable), the different time scales of the signal introduce new and subtle problems in the analysis of conjunction data. As the relativistic effect evolves overtime scales of, say, $10^{5} \mathrm{~s}$ (i.e., longer than the period of gravitational waves considered so far) the orbital contribution to the Doppler shift needs to be determined with great accuracy. Polar motion, earth solid tides and errors in the station locations give important effects to be accounted for. Other contributions come from non-gravitational accelerations, which are a potentially large source of errors in an experiment based on a free flying spacecraft. Fortunately, the large distance from the sun and the nearly constant solar aspect angle make these errors quite small or easily accounted for in the data analysis.

\subsubsection{Study of the Solar Corona}

Tracking interplanetary spacecraft near solar conjunction has provided a wealth of information on the structure of the solar corona and the origin of the solar wind. This powerful tool, in combination with SOHO's ultraviolet coronograph spectrometer, has been used recently to locate the sources of the slow wind, which stems from the stalks, narrow structures of the sun's streamer belt (Woo and Habbal, 1997). The same measurements seem to indicate also that the conventional understanding of the nature of the fast wind needs to be modified. It is likely that in the next years new measurements and observations will be required to confirm the new, emerging views on the solar wind.

The outstanding radio system of Cassini provides a unique opportunity for solar physics as well, without any additional allocation of resources from the spacecraft and the DSN. The effects of the solar plasma on Doppler signals are known with great precision separately for the uplink and the down-link paths, thanks to the multifrequency radio link (Bertotti and Giampieri, 1998). Moreover, the use of higher frequency carriers will strongly reduce the difficulties and the instrumental problems encountered when tracking near the sun.

\subsection{Gravitational Field Measurements and Celestial MECHANICS EXPERIMENTS}

\subsubsection{Scientific Objectives}

2.3.1.1. Introduction. Over the past 35 years, radio Doppler data generated with interplanetary spacecraft have yielded masses and densities for all the planets, except Pluto, as well as masses for all the larger satellites, and higher-order 
gravitational moments are available for the planets and for Jupiter's Galilean satellites (Anderson et al., 1996a,b, 1997a,b). With the addition of Doppler data from the Cassini mission we expect to improve the description of Saturn's gravitational field, as well as to map Titan's second-order field in detail.

If a planet or satellite were perfectly spherical, its total mass would be sufficient to describe its external gravitational field. However all natural bodies deviate from spheres at some level, so what is needed is a general potential function that satisfies Laplace's equation for an arbitrary distribution of mass. With the origin at the center of mass and coordinates $r$ for radius, $\phi$ for latitude, and $\lambda$ for longitude, the standard form for the gravitational potential is written as follows in terms of spherical harmonics and the Legendre functions $P_{n m}$

$$
\begin{aligned}
& V(r, \phi, \lambda) \\
& =\frac{G M}{r}\left[1+\sum_{n=2}^{\infty} \sum_{m=0}^{n}\left(\frac{R}{r}\right)^{n}\left(C_{n m} \cos m \lambda+S_{n m} \sin m \lambda\right) P_{n m}(\sin \phi)\right],
\end{aligned}
$$

where $M$ is the planet or satellite's mass, the external field is referred to a reference radius $R$ (usually the planet's mean equatorial radius, but sometimes the semimajor axis of a reference ellipsoid); $G$ is the gravitational constant. The gravity coefficients $C_{n m}$ and $S_{n m}$ are determined from the Doppler data by iterative linear weighted least squares. A particular coefficient with indices $\mathrm{nm}$ is referred to as a gravity harmonic of order $m$ and degree $n$. Coefficients with $m=0$ are called zonal harmonics, coefficients with $n=m$ are called sectorial harmonics, and the rest are called tesseral harmonics. Zonal harmonics divide the surface of the sphere into $m+1$ zones of latitude, sectorial harmonics into sectors of longitude or "orange slices," and tesseral harmonics into a checker-board pattern.

The outer planets and their larger satellites are effectively in hydrostatic equilibrium. As a result, measured gravity harmonics provide important boundary conditions on their interior structure. If the giant Jovian planets and their satellites were in hydrostatic equilibrium and did not rotate, and if they were not subjected to external forces, their gravitational fields would provide no information on internal structure. However, because the giant planets rotate rapidly, their shapes and gravity fields yield information on the distribution of density with depth. The Galilean satellites and Titan are inuenced by comparable tidal forces from their parent planet, and so both rotation and tides must be accounted for. For the giant planets, the two parameters for shape and rotation are the flattening $f$ and the rotation parameter $q$ defined by,

$$
\begin{aligned}
& f=\frac{a-b}{a}, \\
& q=\frac{\omega^{2} a^{3}}{G M},
\end{aligned}
$$


where $a$ is the planet's equatorial radius, $b$ its polar radius, $\omega$ its rotational angular velocity, and $G$ is the gravitational constant. Even without gravity measurements, the ratio $f / q$ would yield some limited information on interior structure because it has a minimum value of $1 / 2$ for a body with an extreme mass concentration at its center, and a maximum value of 5/4 for a homogeneous body. For a spinning planet in hydrostatic equilibrium, we assume that only the even zonal gravity harmonics $J_{n}=$ $-C_{n 0}(n=2,4,6, \ldots)$ are non-zero. The importance of the gravity coefficients $J_{n}$ is that they are related to the internal density distribution by the following volume integral over the planet's interior

$$
J_{n}=-\frac{1}{M R^{n}} \int_{v} \rho(r, \phi, \lambda) r^{n} P_{n}(\sin \phi) d v \quad(n=2,4,6 \ldots) .
$$

The $J_{n}$ coefficients represent boundary conditions that must be satisfied for any viable interior model. The deep interior stimulates the second zonal harmonic $J_{2}$, while the outer layer is sounded by higher harmonics to a depth of about $3100 \mathrm{~km}$ for Jupiter and $3600 \mathrm{~km}$ for Saturn. For example, in a simple polytrope of index one, a reasonably good first-order approximation for Jupiter and Saturn's outer layers, the pressure $p$ and density $\rho$ are related by,

$$
p=K \rho^{2},
$$

and the constant $K$ is determined from the measured gravity coefficients $J_{2}$ and $J_{4}$ by the expression,

$$
K=-\frac{2 \pi G b^{2}}{35 J_{4}}\left(J_{2}+\frac{q}{3}\right)^{2}
$$

For nonpolytropic models, a general density distribution with depth can be derived from measured gravity coefficients $J_{2}$ and $J_{4}$. The coefficient $J_{6}$ may also be useful, although differential rotation and deep atmospheric winds may complicate its interpretation. With a given density distribution, the pressure can be computed under the assumption of hydrostatic equilibrium, and the temperature follows from the equation of state for the assumed material in the outer layer. 
2.3.1.2. Objectives for Saturn. The scientific objectives at Saturn are to:

- determine the mass of Saturn and its zonal gravitational harmonics, at least through degree six;

- constrain models of Saturn's interior.

The product of the gravitational constant by Saturn's mass, $G M_{\mathrm{S}}$, has been determined to considerable accuracy from Pioneer and Voyager flybys (Campbell and Anderson, 1989). After dividing by $G=6.67259 \times 10^{-20} \mathrm{~km}^{3} \mathrm{~s}^{-2} \mathrm{~kg}^{-1}$ (Cohen and Taylor, 1987), we obtain a total mass $M_{\mathrm{S}}=(5.68464 \pm 0.0003) \times 10^{26} \mathrm{~kg}$. A fundamental constraint on interior models is that this total mass must be contained within an equipotential surface defined by Saturn's mean equatorial radius, $R_{\mathrm{S}}=$ $60,268 \mathrm{~km}$ (Lindal et al., 1985). The shape of the equipotential surface is defined by the zonal harmonics, which also serve as additional boundary conditions on interior models. The current unnormalized values, in units of $10^{-6}$, from Pioneer and Voyager Doppler data are (Campbell and Anderson, 1989)

$$
\begin{aligned}
& J_{2}=16332 \pm 10, \\
& J_{4}=-919 \pm 40, \\
& J_{6}=104 \pm 50 .
\end{aligned}
$$

Current interior models based on these values are nonunique (Guillot et al., 1994), although some fail to satisfy the more rigid observational constraint given by Campbell and Anderson (1989),

$$
\left|0.23 \delta J_{2}+2.20 \delta J_{4}-3.13 \delta J_{6}\right| \leq 1,
$$

where the $\delta$ corrections are with respect to the values of Equations (15)-(17). Because interior models are fundamentally constrained by the gravitational moments, improvements in accuracy by means of Cassini radio Doppler data may yield a better understanding of Saturn's interior. The Cassini mission can provide improved determinations for all three zonal harmonics. Our best estimate of the expected one-sigma accuracy, again in units of $10^{-6}$, is \pm 0.1 for $J_{2}, \pm 0.4$ for $J_{4}$, and \pm 1.5 for $J_{6}$. In addition, the Cassini mission should improve other important parameters, most notably the rotational period of Saturn's magnetic field (10.6549 h) (Davies et al., 1983), its intrinsic power output $\left(8.63 \times 10^{16} \mathrm{~J} \mathrm{~s}^{-1}\right)$ and internal energy flux $\left(2.01 \mathrm{~J} \mathrm{~m}^{-2} \mathrm{~s}^{-1}\right.$ ) (Hanel et al., 1983), the temperature of the atmosphere at the one bar level (134 K) (Lindal et al., 1985), and the mean equatorial radius. These various boundary conditions must be satisfied by solving the nonlinear differential equations for the interior structure (see for example Guillot et al., 1994). Post-Cassini models may lead to a better understanding of the energy transport in Saturn's interior and consequently to a better understanding of its cooling history and evolution. 
2.3.1.3. Objectives for Titan. The Radio Science gravitational scientific objectives at Titan are to:

- determine the mass of Titan and its low degree and order gravitational harmonics $\left(J_{2}, C_{22}\right)$;

- measure the tidal variation of Titan's gravitational quadrupole moments;

- constrain models of Titan's interior.

a) Determine Titan's mass and low degree and order gravity field: The most accurate determination of Titan's mass $M_{\mathrm{T}}$ has been obtained by a combination of radio Doppler data from Pioneer 11, Voyager 1 and Voyager 2 (Campbell and Anderson, 1989). Voyager 1 encountered Titan at a closest approach distance of $6500 \mathrm{~km}$. Pioneer 11 and Voyager 2 encountered Titan at much farther distances of 363,000 and $666,000 \mathrm{~km}$, respectively. The best current value of GMT is $8978.2 \pm$ $1 \mathrm{~km}^{3} \mathrm{~s}^{-2}$.

None of the Titan encounters has yielded any information on gravitational harmonics beyond the zero-degree mass term. The values of $J_{2}$ and $C_{22}$ can be derived theoretically by assuming that Titan's low degree and order gravitational field reects the satellite adjustment to the perturbing potentials excited by Titan's rotation and by Saturn's tides. The static part of the perturbing potential produces a permanent deformation of the body with coefficients (Rappaport et al., 1997)

$$
\begin{gathered}
J_{2}=\frac{5 k_{\mathrm{f}}}{6} \frac{M_{\mathrm{S}}}{M_{\mathrm{T}}}\left(\frac{R_{\mathrm{T}}}{a_{\mathrm{T}}}\right)^{3}=\left(\frac{k_{\mathrm{f}}}{1.5}\right) 0.49 \times 10^{-4}, \\
C_{22}=\frac{k_{\mathrm{f}}}{4} \frac{M_{\mathrm{S}}}{M_{\mathrm{T}}}\left(\frac{R_{\mathrm{T}}}{a_{\mathrm{T}}}\right)^{3}=\left(\frac{k_{\mathrm{f}}}{1.5}\right) 0.15 \times 10^{-4},
\end{gathered}
$$

in Titan's principal axes of inertia frame. In Equations (19) and (20), $R_{\mathrm{T}}$ is Titan's equatorial radius, $a_{\mathrm{T}}$ is Titan's orbital semi-major axis, and $k_{\mathrm{f}}$ is the fluid Love number. For a homogeneous uid, $k_{\mathrm{f}}=3 / 2$, but it could be as much as two times smaller if there were substantial central condensation in the interior of Titan. Note that for all possible internal mass distributions in hydrostatic equilibrium, $J_{2}$ is exactly $10 / 3$ of $C_{22}$.

An order of magnitude calculation (Rappaport et al., 1997) indicates that measurements of the Doppler frequency shift in the two-way radio signal between the Cassini spacecraft and a ground station will allow an excellent determination of $J_{2}$ and $C_{22}$ with absolute accuracy of order

$$
\sigma_{2}=\frac{c b v}{G M_{\mathrm{T}}}\left(\frac{b}{R_{\mathrm{T}}}\right)^{2} \sigma_{y} \simeq 4 \times 10^{-8},
$$

where we assume the distance at closest approach $b$ is twice Titan's radius $R_{\mathrm{T}}, c$ is the speed of light, the spacecraft's speed $v \simeq 5 \mathrm{~km} \mathrm{~s}^{-1}$, and the Allan deviation of the Cassini radio signal over a typical Doppler integration time is $\sigma_{y} \simeq 10^{-14}$. 
Equation (21) is consistent with results for Ganymede obtained with the Galileo spacecraft (Anderson et al., 1996b).

An analytical covariance analysis (Rappaport et al., 1997) suggests that $J_{2}$ and $C_{22}$ can be measured with the Cassini mission to an absolute accuracy between $10^{-8}$ and $10^{-9}$, which is consistent with the rough estimate of Equation (21). That covariance analysis allows us to estimate the accuracy afforded by various flyby geometries. Favorable conditions include a small impact parameter, a small flyby velocity, a velocity at closest approach not too far from the line of sight, and an asymmetric flyby with respect to Titan's principal axes of inertia.

b) Determine tidal variation of Titan's quadrupole moments: In addition to a permanent deformation, Titan must undergo a periodic deformation in response to the changing tidal force caused by the significant eccentricity of Titan's orbit. The periodic tidal perturbation has a frequency equal to Titan's orbital angular frequency, and Titan's response is fundamentally different from its static response. While Titan responds more or less as a fluid body to the static potential, it responds as an elastic body to the periodic potential.

The tidal variation of Titan's quadrupole moment is described in terms of (Rappaport et al., 1997)

$$
\begin{aligned}
\Delta J_{2} & =\frac{3 k_{2}}{2} \frac{M_{\mathrm{S}}}{M_{\mathrm{T}}}\left(\frac{R_{\mathrm{T}}}{a_{\mathrm{T}}}\right)^{3} e \cos f=\left(\frac{k_{2}}{0.015}\right) \times 2.58 \times 10^{-8} \cos f, \\
\Delta C_{22} & =\frac{3 k_{2}}{4} \frac{M_{\mathrm{S}}}{M_{\mathrm{T}}}\left(\frac{R_{\mathrm{T}}}{a_{\mathrm{T}}}\right)^{3} e \cos f=\left(\frac{k_{2}}{0.015}\right) \times 1.29 \times 10^{-8} \cos f,
\end{aligned}
$$

where $e=0.029$ is Titan's orbital eccentricity, $\mathrm{f}$ is its true anomaly, and $k_{2}$ is the Love number of degree 2 (Love, 1906). For an incompressible body with uniform density $\rho$ (for Titan, $\rho=1.88 \mathrm{~g} \mathrm{~cm}^{-3}$ ) and elastic shear modulus (or rigidity) $\mu$,

$$
k_{2}=\frac{3 / 2}{1+19 \mu R_{\mathrm{T}} / 2 G M_{\mathrm{T}} \rho} .
$$

While deviations from incompressibility and homogeneous density require only small corrections to Equation (24) (Kaula 1964), a varying rigidity has important consequences (Cassen et al., 1982).

The rigidity of Titan is highly uncertain. For a range of rigidity's values from $\mu=4 \times 10^{10}$ dyne $\mathrm{cm}^{-2}$ (appropriate for an icy body) to $\mu=7 \times 10^{11}$ dyne $^{-2}$ (appropriate for a rocky body) we obtain a range of values of $k_{2}$ from 0.22 to 0.015 . It follows that the amplitudes of variations of $J_{2}$ and $C_{22}$ (estimated in Equations (22) and (23) to be $2.6 \times 10^{-8}$ and $1.3 \times 10^{-8}$, respectively) could be as much as 15 times larger. From covariance analysis results we conclude that a determination of $k_{2}$ to within 0.05 absolute accuracy is possible.

c) Constrain models of Titan's interior: The assumption of hydrostatic equilibrium will be tested by determining $J_{2}$ and $C_{22}$ (Hubbard and Anderson, 1978). 
Indeed, important departures from hydrostatic equilibrium would invalidate the relationship between $J_{2}$ and $C_{22}$.

If the assumption of hydrostatic equilibrium is verified, then the value of $k_{\mathrm{f}}$ will provide the value of the greatest moment of inertia $C$ from the Radau equation (see Hubbard, 1984)

$$
\frac{C}{M_{\mathrm{T}} R_{\mathrm{T}}^{2}}=\frac{2}{3}\left[1-\frac{2}{5}\left(\frac{5}{k_{\mathrm{f}}+1}-1\right)^{1 / 2}\right] .
$$

The value of $C /\left(M_{\mathrm{T}} R_{\mathrm{T}}^{2}\right)$ depends on the degree of differentiation of the body. It will be compared to values predicted by various models of internal structure (e.g., Sohl et al., 1995).

Finally, the value of $k_{2}$ will be used to distinguish between volatile-rich and volatile-poor models of Titan (Stevenson, 1992). The above-mentioned range of $k_{2}$ values (from 0.22 to 0.015 ) represent the extreme range from volatile rich to volatile poor models. Volatile-rich models are so-called because they have a deep, internal, water-ammonia ocean (Lunine and Stevenson, 1987; Cynn et al., 1989; Grasset and Sotin, 1996). These models are supported by our ideas of the formation of satellites, but imply large amounts of methane which could be present in part as surface hydrocarbon oceans. These seem inconsistent with a primordial origin of Titan's orbital eccentricity (Sohl et al., 1995). Indeed, oceanic tidal friction would have circularized Titan's orbit by now.

On the other hand, the volatile-poor models, in which Titan's mantle is completely solid, are consistent with a primordial orbital eccentricity, but have cosmogonic problems described by Stevenson (1992).

These problems lead us to briey re-examine underlying assumptions concerning Titan's orbital eccentricity and Titan's formation; such ideas can be tested by the gravity measurements.

Early work by Sagan and Dermott (1982) implicitly assumed that Titan's orbital eccentricity is primordial. These authors identified two scenarios consistent with the persistence of eccentricity in the presence of tidal dissipation: either Titan is covered by a nearly global methane ocean deeper than $400 \mathrm{~m}$, or there is no methane ocean on the surface. The first picture was favored because such an ocean would resupply the atmosphere in methane and hence balance atmospheric methane destruction by photolysis, which will remove the present atmospheric inventory in 107-108 years (Yung et al., 1984).

However, the speculated global ocean was dispelled by radar (Grossman and Muhleman, 1992; Muhleman et al., 1990, 1992) and infrared (Griffith, 1993; Lemmon et al., 1993; Coustenis et al., 1994; Han and Owen, 1994; Smith et al., 1994) observations of Titan, although these allow for seas or lakes.

Sears (1995), using a more sophisticated tidal dissipation model than Sagan and Dermott (1982), concluded that even ignoring land masses, the present orbital eccentricity of Titan requires that the depth of any global ocean be at least $500 \mathrm{~m}$. 
Dermott and Sagan (1995) found that confining the fluid on Titan to a number of disconnected seas or crater lakes greatly extends the damping timescale of Titan's orbital eccentricity. One problem with this idea is that restricted seas and crater lakes may not contain sufficient amount of methane to resupply the atmosphere over the age of the solar system (Lunine, 1996). Stevenson (1992) suggested that the hydrocarbon ocean is stored in porous spaces and caverns within the upper crust of Titan. This model may reconcile the need for a reservoir of hydrocarbons with the tidal argument against a global ocean, though it demands a rather porous crust.

Lorenz et al. (1997) proposed the idea that Titan's atmosphere may be unstable and exists only episodically in its present extensive state. This model may be able to solve the eccentricity problem if Titan's surface remains frozen for long periods of time corresponding to epochs in which the atmosphere is collapsed.

Finally, the possibility remains that Titan's orbital eccentricity was produced recently (less than several hundred million years ago) by a large impact, though few bodies larger enough to do so are expected to exist on highly eccentric orbits.

If Titan formed around Saturn, an ammonia-water layer may have formed inside Titan and persisted (Grasset and Sotin, 1996).

However, Prentice $(1980,1984)$ suggested that Titan could be a captured moon of Saturn. Lewis and Prinn (1980) showed that only small amounts of methane and ammonia were present in the solar nebula, so that Titan could have formed as a volatile-poor body. If produced from solar nebula material in the vicinity of Saturn's orbit, then Zahnle et al. (1992) and Griffith and Zahnle (1995) showed that volatiles could have been delivered to Titan by comets. Impact-driven chemistry (Jones and Lewis, 1987) would have led to production of organic compounds in a manner which may or may not be compatible with Titan's atmosphere and surface composition, but some comets themselves contain significant complements of organic compounds which may survive impact on Titan.

2.3.1.4. Objectives for the Icy Satellites. The radio science gravitational scientific objectives at the icy satellite are to:

- determine the gravity fields of Enceladus and Rhea $\left(J_{2}, C_{22}\right)$;

- determine the masses of Mimas, Tethys, Dione, Hyperion and Phoebe.

a) Enceladus' gravity field: Enceladus is a special focus of interdisciplinary science in the Cassini mission. Indeed, two major objectives of the Cassini Satellite Surface Working Group are to acquire optical remote sensing observations of Enceladus and to measure its gravity field.

This interest is explained by the intriguing characteristics of Enceladus, among which figures the remarkably high and uniform albedo and the presence of old and recent terrains. The mean geometric albedo is close to unity, and the total range of albedo over the surface is $20 \%$. This is even more intriguing considering that Enceladus topography contains both old cratered terrains dating from the period of heavy bombardment and smooth terrains recently resurfaced, indicating endogenic 
activity. Buratti (1988) investigated the photometric properties of Enceladus and concluded that the satellite is completely covered by a young, bright surface layer. Buratti argues that the E-ring, whose thickness peaks at the orbit of Enceladus, is the most likely source for this layer, and that the E-ring itself is probably the result of active surface processes on Enceladus. Alternatively, the bright surface of Enceladus may result from dissipation associated with tidal interactions, perhaps with Dione.

Enceladus's mean radius is $\simeq 250 \mathrm{~km}$. How can such a small satellite have undergone substantial evolution? This may be due to the fact that ammonia ice, which plays an important role in lowering the melting point, may have been incorporated into Enceladus. Evidently, the surface properties alone are insufficient to provide information on the interior composition. To understand the nature and history of Enceladus and the other Saturn's satellites, modeling of the interiors and of the thermal evolutions is in order.

As far as Enceladus is concerned, even the most basic parameter, i.e., the mass density, is very poorly known. Indeed, the mass of Enceladus is know with about $50 \%$ accuracy. Assuming $G M_{\mathrm{E}}=4.9 \mathrm{~km}^{3} \mathrm{~s}^{-2}$ (Campbell and Anderson, 1989), hydrostatic equilibrium, and a value of the fluid Love number equal to half its value for a homogeneous body, we obtain $J_{2}=0.0056$ and $C_{22}=0.0017$.

A determination of the mass of Enceladus and of its harmonic coefficients of degree 2 from data acquired during a targeted flyby will allow us to determine the greatest moment of inertia from Equation (25), and hence to constrain models of internal structure.

b) Rhea's gravity field: With a radius of $765 \mathrm{~km}$, Rhea is the largest satellite of Saturn after Titan. This is the reason why Rhea was selected for gravity field determination. Recently, Anderson et al. (1996a, 1996b) came to the surprising but inescapable conclusion that both Io and Ganymede contain large metallic cores. It will be very interesting to find out whether the smaller Rhea is differentiated and to compare its internal structure to that of Titan and Enceladus. In this regard, note that distinct terrain ages and endogenic resurfacing were suggested by Plescia and Boyce's (1982) discovery that two polar regions have retained large craters while they are absent in another region near the equator.

c) Icy satellites' masses: The objective is to determine the masses of the icy satellites (especially Mimas, Tethys, Dione, Hyperion and Phoebe) in order to determine their mean density to high accuracy, and hence constrain their bulk composition. This is essential for an improved understanding of their dynamical behavior and evolution. Tyler et al. (1981) determined the masses of Titan and Rhea from Voyager 1 radio science measurements at Saturn, and Voyager 2 permitted Tyler et al. (1982) to determine the masses of Tethys and Iapetus. Using the mass of Tethys in combination with the theory of the Tethys-Mimas resonance, they derived the mass of Mimas. Campbell and Anderson (1989) used the combined data set of Pioneer and Voyager data to redetermine the masses of Tethys, Rhea, Titan and 
TABLE I

Masses of the large icy satellites of Saturn.

\begin{tabular}{|c|c|c|c|c|c|}
\hline Satellite & $\begin{array}{l}\text { Mass } \\
\left(\times 10^{23} \mathrm{~g}\right)\end{array}$ & Reference & $\begin{array}{l}\text { Mean Radius } \\
\left(\times 10^{5} \mathrm{~cm}\right)\end{array}$ & Reference & $\begin{array}{l}\text { Density } \\
\left(\mathrm{g} \mathrm{cm}^{-3}\right)\end{array}$ \\
\hline \multirow[t]{2}{*}{ Mimas } & $0.46 \pm 0.05$ & $\begin{array}{l}\text { Tyler } \text { et al., } 1982 \\
\quad \text { (derived) }\end{array}$ & $196 \pm 3$ & $\begin{array}{l}\text { Smith et al., } \\
1982\end{array}$ & $1.46 \pm 0.23$ \\
\hline & $0.375 \pm 0.009$ & Kozai, 1976 & $198.6 \pm 0.6$ & $\begin{array}{l}\text { Davies et al., } \\
1996\end{array}$ & $1.14 \pm 0.04$ \\
\hline \multirow[t]{2}{*}{ Enceladus } & $0.74 \pm 0.36$ & Kozai, 1976 & $250 \pm 10$ & $\begin{array}{l}\text { Smith et al., } \\
\quad 1982\end{array}$ & $1.13 \pm 0.69$ \\
\hline & $0.74 \pm 0.36$ & Kozai, 1976 & $249.4 \pm 0.3$ & $\begin{array}{l}\text { Davies et al., } \\
\quad 1996\end{array}$ & $1.14 \pm 0.56$ \\
\hline \multirow[t]{2}{*}{ Tethys } & $7.55 \pm 0.90$ & Tyler et al., 1982 & $530 \pm 10$ & $\begin{array}{l}\text { Smith et al., } \\
\quad 1982\end{array}$ & $1.21 \pm 0.21$ \\
\hline & $6.22 \pm 0.13$ & Kozai, 1976 & $529.8 \pm 1.5$ & $\begin{array}{l}\text { Davies et al. } \\
1996\end{array}$ & $1.00 \pm 0.03$ \\
\hline \multirow[t]{2}{*}{ Dione } & $10.52 \pm 0.33$ & Kozai, 1976 & $560 \pm 5$ & $\begin{array}{l}\text { Smith et al., } \\
\quad 1982\end{array}$ & $1.43 \pm 0.08$ \\
\hline & $10.52 \pm 0.33$ & Kozai, 1976 & $560 \pm 5$ & $\begin{array}{l}\text { Davies et al. } \\
\quad 1996\end{array}$ & $1.43 \pm 0.08$ \\
\hline \multirow[t]{2}{*}{ Rhea } & $24.9 \pm 1.5$ & Tyler et al., 1981 & $760 \pm 5$ & $\begin{array}{l}\text { Smith et al., } \\
\quad 1982\end{array}$ & $1.33 \pm 0.11$ \\
\hline & $23.1 \pm 0.6$ & $\begin{array}{l}\text { Campbell and } \\
\text { Anderson, } 1989\end{array}$ & $764 \pm 4$ & $\begin{array}{l}\text { Davies et al., } \\
1996\end{array}$ & $1.24 \pm 0.05$ \\
\hline \multirow[t]{2}{*}{ Iapetus } & $18.8 \pm 1.2$ & Tyler et al., 1982 & $730 \pm 10$ & $\begin{array}{l}\text { Smith et al., } \\
\quad 1982\end{array}$ & $1.15 \pm 0.12$ \\
\hline & $15.9 \pm 1.5$ & $\begin{array}{l}\text { Campbell and } \\
\text { Anderson, } 1989\end{array}$ & $718 \pm 8$ & $\begin{array}{l}\text { Davies et al., } \\
\quad 1996\end{array}$ & $1.02 \pm 0.13$ \\
\hline
\end{tabular}

Iapetus, but recommended the earlier ground-based determination (Kozai, 1957) for Tethys. No mission has yet yielded the masses of Mimas, Enceladus, Dione, Hyperion and Phoebe.

Mass, size and density for the intermediate-size satellites are listed in Table I. For each satellite, the density on the first line is computed from the mass determined or recommended by Tyler et al. (1982) and the mean radius determined by Smith $e t$ al. (1982) from Voyager images. The density on the second line is computed from the mass determined or recommended by Campbell and Anderson (1989) and the mean radius from Davies et al. (1996).

Phoebe, on an inclined, retrograde orbit, may be a captured object. Determining the density of Phoebe is important to find out whether or not Phoebe is asteroidal in nature. 


\subsubsection{Techniques}

2.3.2.1. Saturn. Cassini will orbit Saturn at a fairly wide range of periapsis radii between 1.3 and $7.3 R_{\mathrm{S}}$, at inclinations between $0^{\circ}$ and $85^{\circ}$, and with a wide range of orbital periods from 3 months to 8 days. By fitting a second degree and order gravitational field, in addition to $J_{4}$ and $J_{6}$, to the radio Doppler data, the mapping of Saturn's gravitational field will be complete.

Flybys of Saturn on previous NASA missions occurred at periapsis radii of 1.34 $R_{\mathrm{S}}$ for Pioneer 11, 3.05 $R_{\mathrm{S}}$ for Voyager 1, and $2.67 R_{\mathrm{S}}$ for Voyager 2 . However, the Pioneer 11 Doppler noise is a factor of 260 times that expected from the Cassini $\mathrm{X}$-band data, while the Voyager noise is a factor of 12 larger than the expected Cassini noise. Given the improved Doppler accuracy for Cassini, and the expected smaller non-gravitational accelerations, plus the advantage of multiple orbits of Saturn, it is realistic to expect a factor of 100 improvement over Pioneer 11 and Voyager in our knowledge of Saturn's gravitational moments.

The radio Doppler data may be complemented by the reduction and analysis of imaging data for the study of the orbital mechanics of smaller inner satellites and eccentric ringlets. It was demonstrated during the Voyager mission that such orbital data can place independent constraints on Saturn's gravitational field (Nicholson and Porco, 1988), which when combined with the radio Doppler data, provide significantly better determinations of the higher-order gravitational moments.

2.3.2.2. Satellites. The gravity fields of Titan, Enceladus and Rhea will be determined by the same type of global technique as used for Saturn.

The tidal variation of the low degree and order gravity field will be measured from independent determinations of $J_{2}$ and $C_{22}$ obtained from flybys occurring near Titan's periapsis and apoapsis.

\subsubsection{Major Requirements}

2.3.3.1. Saturn Requirements. The major requirement for the determination of the gravitational moments is to obtain coherent $\mathrm{X}$-band Doppler data over an interval of $5 \mathrm{~h}$, centered on the closest approach in each Saturn orbit. Outside of closest approach, the data do not need to be continuous, but we require as nearly continuous data as possible for an interval of $\pm 4 \mathrm{~h}$. Although our covariance analysis, based on an assumed Allan deviation of $2 \times 10^{-14}$ at a $1000 \mathrm{~s}$ integration time, is valid for $\mathrm{X}$-band tracking only, the determination of Saturn's gravitational field will most likely be improved further with a dual-band radio capability at X-and Ka-bands. The most significant enhancement will be the relative insensitivity of the data noise to solar longation angle, thereby enabling a high quality moment determination on every orbital revolution.

The best orbits of opportunity are those with periapsis radii of less than $4.5 R_{\mathrm{S}}$. About 40 such opportunities are anticipated. The first and closest orbit at $1.3 R_{\mathrm{S}}$ will receive the highest priority. A wide range of inclination angles is practically 
assured because of the mission requirement to raise the inclination to a near polar orbit during orbits 34-59.

In principle, we require that there be no spacecraft maneuvers or momentum dumps of the reaction wheels during the two days of data acquisition for Saturn radio science. In practice, this may not be achieved. But when it is, we anticipate that the RSS total unmodeled acceleration by spacecraft subsystems, particularly the attitude and articulation control subsystem and the propulsion module subsystem, when inactive, will be less than $5 \times 10^{-14} \mathrm{~km} \mathrm{~s}^{-2}$, about 100 times smaller than comparable acceleration noise on the Voyager spacecraft.

\subsubsection{Titan Requirements.}

a) Tour requirements and flybys selection: The tour must contain at least four Titan flybys in the following conditions:

- The spacecraft must not be occulted by Titan during $\pm 2 \mathrm{~h}$ around closest approach. This is to allow us to track the spacecraft.

- The distance at closest approach $b$ must be such that $1.5 \leq b / R_{\mathrm{T}} \leq 2$. As a matter of fact, a flyby such that $b<1.5 R_{\mathrm{T}}$ would require firing the thrusters, which would introduce noise in the data. On the other hand, given that the accuracy of the determination is proportional to $\left(b / R_{\mathrm{T}}\right)^{3}$ (see Equation (21)), there is a rapid loss of sensitivity as the flyby's altitude is raised.

- Two flybys must occur near Titan's periapsis, and include one flyby at low inclination with respect to Titan and another one at high inclination. Two other flybys must occur near Titan's apoapsis, with the same inclination requirement. The inclination requirement will allow a good separation of $J_{2}$ from $C_{22}$. The Titan's periapsis and apoapsis requirement will allow us to determine the tidal variations of Titan's quadrupole moments.

- The flybys should occur far from solar conjunction to minimize the effects of plasma noise. For example, plasma noise increases by two orders of magnitude between sun-earth-spacecraft angles of $120^{\circ}$ and $7^{\circ}$.

- Goldstone viewing by the deep space station DSS-25 is desirable because the use of Ka-band afforded by this station reduces the plasma noise by one order of magnitude.

b) Tracking requirements: Tracking data must be acquired continuously for \pm 2 $\mathrm{h}$ around the time of closest approach.

\subsubsection{Icy Satellite Requirements.}

- The determination of Enceladus and Rhea's gravity fields requires that one targeted flyby of each satellite be dedicated to gravity field measurements.

- Mass determination can be performed with non-targeted flybys which have a distance at closest approach smaller than $50,000 \mathrm{~km}$ and preferably smaller than $25,000 \mathrm{~km}$. Tracking data of Phoebe will be acquired during the Phoebe flyby, 19 days before Saturn orbit insertion. 


\subsection{RING OCCULTATION EXPERIMENTS}

\subsubsection{Objectives}

Voyager observation of Saturn's rings in 1980 provided the only radio occultation observation available to date of the remarkable Saturnian ring system (Tyler et al., 1980). All features of the main ring system were probed using coherent 3.6 and 13-cm wavelengths sinusoidal signals. Cassini ring observations will significantly expand on the Voyager observations, primarily taking advantage of new three simultaneous wavelengths capability $(0.94,3.6$, and $13 \mathrm{~cm}$, or Ka-, X-, and S-bands, respectively) as well as multiple occultation opportunities at large, intermediate, and small ring opening angle $B$.

2.4.1.1. Ring Structure and Physical Properties. Analysis of the Voyager radio occultation observations has contributed a wealth of information regarding radial ring structure (Tyler et al., 1983; Marouf et al., 1986; see also maps in Rosen, 1989), the particle size distribution of several broad ring features (Marouf et al., 1983; Zebker et al., 1985), physical ring thickness (Marouf et al., 1982; Zebker and Tyler, 1984; Zebker et al., 1985), and ring dynamics (Marouf and Tyler, 1986; Marouf et al., 1987; Gresh et al., 1986; Rosen and Lissauer, 1988; Rosen et al., 1991a,b).

A small ring opening angle $B=5.9^{\circ}$ (angle between a unit vector in the Earth direction and the ring plane) at the time of the Voyager observations increased the effective path length of the radio signal through the rings by a factor of about 10 relative to its normal value. The increased length enhanced sensitivity to regions of small to moderate optical depth (Rings C, Cassini Division, and Ring A), but accentuated signal attenuation over most of Ring B as well as other optically thick ring features (Tyler et al., 1983).

Accurate, high spatial resolution, multiple wavelengths characterization of ring structure and of its variability with ring longitude, ring opening angle, and time are major objectives of the Cassini radio science ring observations. The characterization is at the heart of understanding of ongoing physical and dynamical processes and is of prime importance for eventual understanding of ring origin and evolution.

Radio occultation opportunities implemented early in the Cassini tour when the rings are nearly fully open $\left(B \approx 20-24^{\circ}\right)$ will overcome a major Voyager limitation, allowing for the first time probing at radio wavelengths of the optically thick Ring B, which dominates the overall mass of the ring system. The optimized near-diametric geometry of these occultations will yield an order of magnitude resolution improvement in mapping the radial structure of ring features compared with Voyager. Other occultations later in the mission at intermediate and small ring opening angle B will provide complementary information regarding vertical ring structure, optical depth profiles of tenuous ring features, and the variability of ring structure with longitude and time. 
The Cassini observations will also determine the particle size distribution of major ring features, including Ring B, over the millimeters to tens of meters size range. Knowledge of the size distribution constrains the surface mass density of a ring feature if the ring material is assumed to be solid water ice. Alternatively, the size information may be used to constrain the material density of ring particles if independent estimates of the surface mass density are available from analysis of local dynamical features.

The multi-wavelength observations will also yield high spatial resolution information regarding the abundance of millimeter to decimeter size particles within local ring features, a unique capability of the radio occultation observations. Of particular interest is mapping the possible variability in the abundance of this particle population within dynamically active ring features (density and bending waves, wakes of embedded satellites, resonant edges, etc.). Interesting sorting of particle sizes across narrow ring features has been noted in the Voyager observations (Tyler et al., 1983; Marouf et al., 1986) and has motivated the proposal of innovative ring models to explain the observations (Eshleman, 1983; Eshleman et al., 1983; Michel, 1982).

Specific objectives include:

- High-resolution profiling of radial ring structure and characterization of its variability with wavelength, longitude, ring opening angle, and time. Profiling of the relative abundance of millimeter to decimeter radius particles and characterizing of their variability across resolved ring features.

- Determination of the full particle size distribution over the approximate size range $1 \mathrm{~mm}$ to $20 \mathrm{~m}$ of broad ring features that can be resolved in the spectra of the near-forward scattered signal. Determination of the vertical ring structure, of the physical ring thickness, and of the particle packing fraction within such features.

- Determination of ring surface mass density, ring viscosity, and bulk density of particle material. Characterization of the variability of these parameters among global ring features $(\mathrm{A}, \mathrm{B}, \mathrm{C}, \ldots)$ and within local broad features.

- Characterization of the dust abundance within the main rings, especially in dynamically active regions, through collective analysis of radio, stellar, and solar ring occultation observations.

\subsubsection{Ring Kinematics and Dynamics.}

a) Geometry of the ring system: The Voyager 1 and 2 encounters provided a detailed view of Saturn's complex ring system, with its density and bending waves and its non-circular features, and gave some indications about the composition and particle size distributions of the rings, but additional observations are required to refine the determination of ring orbits, the planetary gravitational field, and the orientation of the ring plane. This is in part because a long time baseline between observations is required for accurate measurement of the precession rates of nearly circular ring features, and also because a suite of different viewing geometries is 
needed to constrain the direction of the planet's spin axis. An important goal of the radio science ring occultation observations is to determine the absolute radius scale of the ring system and the instantaneous direction of Saturn's pole to high accuracy.

Surprisingly, the radius scale of Saturn's rings has a much larger uncertainty - about $1 \mathrm{~km}$ - than the Uranian rings, whose orbital radii are accurate to a few hundred meters or less. This is because the Uranian rings have been observed using dozens of stellar occultations over a twenty-year period, providing a dense set of measurements of ring occultation event times. In contrast, stellar occultations by Saturn are much more difficult to observe because of the brightness of the rings in reflected sunlight. Ground-based observations of the 1989 occultation of a very bright star, 28 Sgr (French et al., 1993; Hubbard et al., 1993), and subsequent occultations observed from the Hubble Space Telescope (HST) (Elliot et al., 1992; Bosh and Olkin, 1996), have provided the only post-Voyager occultations with sufficient Signal-to-Noise Ratio (SNR) to be used for refinement of the ring orbits.

An accurate radius scale for Saturn's rings is essential for detailed dynamical investigations of the rings, as well as accurate inter-comparisons of individual occultation profiles. Since the radial dependence of eccentric ringlet free precession rates is governed by the gravitational harmonics $J_{2}, J_{4}, J_{6}, \ldots$, (Longaretti and Borderies, 1991; Borderies-Rappaport and Longaretti, 1994) a long time series of precise measurements of non-circular features can be used to determine the loworder gravitational harmonics of Saturn. As pointed out above, these give important information about the internal mass distribution of the planet.

The determination of the radius scale is strongly coupled to the assumed pole direction of the mean ring plane, and thus we must solve for both from the occultation observations. From their analysis of the $28 \mathrm{Sgr}$ and Voyager observations, French et al. (1993) concluded that the pole direction had shifted measurably between the 1980/1981 Voyager epochs and the stellar occultation in 1989, and they interpreted this as evidence for precession of Saturn's pole. Although the solar torque exerted on Saturn itself is quite small, the Sun also exerts a torque on the satellites, principally Titan, which effectively increases the $J_{2}$ of the system by a factor of $\simeq 4$ and reduces the precession period by a comparable factor. They found that the rate of motion of Saturn's pole on the sky was $0.86 \pm 0.31$ times the predicted rate of $0.339^{\prime \prime}$ per year. The principal uncertainty in the theoretical value is Saturn's moment of inertia. By incorporating the full set of Cassini occultation observations of the rings, both from radio science measurements and from stellar occultations observed by other Cassini instruments, we have the prospect of greatly improving the accuracy of the precession rate of the pole, with the ultimate goal of determining the principal moment of inertia of Saturn to within a few percent.

For earth-based stellar occultations, the a priori uncertainty in the stellar position relative to the planet is relatively large, and least-squares fits for the occultation geometry must include the offset between the star and planet as a pair of free 
parameters. For spacecraft occultations, the analogous uncertainty is in the spacecraft location with respect to the planet's center. Errors in the assumed spacecraft trajectory map directly into errors in the derived ring plane radius scale. For this reason, we place a very high value on accurate reconstruction of Cassini's trajectory.

b) Ring morphology: A major goal of ring dynamics studies is to explain the presence of nonaxisymmetric features such as eccentric ringlets and density waves, and nonequatorial features such as inclined ringlets and bending waves. Since the Keplerian shear, the differential precession of periapses and nodes, and viscous diffusion tend to erase these features on short time scales, the existing ones must be dynamically maintained.

Satellites are responsible for shaping a number of morphological features. They give rise to density waves (Goldreich and Tremaine, 1978a,b, 1979a; Shu, 1984; Borderies et al., 1986; Longaretti and Borderies, 1986) and bending waves (Shu et al., 1983), open gaps (Goldreich and Tremaine, 1978a; Borderies et al., 1982, 1988), shepherd rings (Goldreich and Tremaine, 1979c; Hanninen and Salo, 1994, 1995; Goldreich et al., 1995), excite eccentricities and inclinations in narrow rings (Goldreich and Tremaine, 1981; Borderies et al., 1983b, 1984a), create ring arcs (Goldreich et al. 1986), and sometimes perturb rings by penetrating them (Borderies et al., 1983c).

The above effects of satellites on rings require that particles behave in a collective manner, which is insured by the ring self-gravity and viscous stresses. Self-gravity is invoked to explain the rigid precession of eccentric and inclined ringlets (Goldreich and Tremaine, 1979b; Borderies et al., 1983a, b). Viscous stresses can lead to instabilities (Lin and Bodenheimer, 1981; Lukkari, 1981; Ward, 1981; Borderies et al., 1985) or over-stabilities (Longaretti and Rappaport, 1995).

The objectives are:

- To test theoretical models for density waves, bending waves, shepherding, excitation of eccentricities and inclinations, ring arcs, precession of elliptical and inclined ringlets, viscous instabilities, etc.

- To identify profiles of gravitational wakes of embedded satellites (Showalter et al., 1986; Marouf et al., 1986; Marouf and Tyler, 1986). Characterize the evolution of profile morphology with longitude and its dependence on background optical depth. Determine the masses and orbits of identified satellites.

- To explain the presence in the $\mathrm{C}$ ring and the Cassini Division of several ringlets which are not associated with any known satellite. Study their interaction with the surrounding rings (Rappaport, 1998).

- To explain the presence in the B ring of non-axisymmetric features which are not associated with any known satellite (Lane et al., 1982; Borderies et al., 1984b).

c) Ring evolution: Angular momentum and energy are transferred between the rings and the satellites with which they interact (Goldreich and Tremaine, 1980). The small satellites outside the main rings of Saturn are repelled outwards over a time scale of a few tens of millions years. This short-time scale suggests that 
the interaction between rings and satellites includes accretion and disruption of satellites and that Saturn's rings as we observe them now may be recent. Alternatively, ring/satellite systems may be maintained by effects that we do not understand yet.

A key parameter in modeling this evolution is the ring's viscosity. This parameter has been measured indirectly from the damping of density and bending waves (Cuzzi et al., 1981; Lane et al., 1982; Lissauer et al., 1982; Shu et al., 1983). Cassini will allow us a more direct determination (Borderies, 1992).

The objectives are:

- To characterize and model viscous stresses.

- To characterize the transport of angular momentum and energy within the rings and their transfer between rings and satellites.

\subsubsection{Techniques}

Observables during a ring occultation experiment can be derived from the effects of ring material on a sinusoidal signal linking the spacecraft and an earth receiving stations of the DSN (Marouf et al., 1982). Temporal coherence of the radio link is ensured through the use of an ultrastable oscillator (USO) onboard the spacecraft and an atomic frequency standard at the ground receiving station. During an occultation, Cassini generates and transmits through the rings three coherent sinusoidal signals (wavelength of $0.94,3.6$, and $13 \mathrm{~cm}$, or Ka-, X-, and S-bands, respectively) using the USO as a common reference for all three signals. The coherency of the signals allows measurement on the ground of the complex amplitude (magnitude and phase) of the perturbed signals.

Modeled as a discrete random medium, the collective effects of ring particles on an incident sinusoidal signal are well characterized (Marouf et al., 1982; Tyler et al., 1983; Marouf et al., 1983; Simpson et al., 1984; Zebker et al., 1985; Marouf et al., 1986; Tyler, 1987; Gresh et al., 1989). Two main signal components may be identified and separated in the spectrum of the observed perturbed signal. The first is the direct or coherent signal, a sinusoidal component whose amplitude and phase can be measured relative to the corresponding values of the incident sinusoid. The direct signal characterizes the average effect of ring particles on the incident signal. The time history of its amplitude and phase provides information regarding detailed radial ring structure. The differential amplitude and phase of two signals of wavelength $\lambda_{1}$ and $\lambda_{2}$ provide information about the relative abundance of particles of radius $a$ determined by $\lambda_{1}, \lambda_{2}$, and the refractive index of particle material (Marouf et al., 1982, 1983).

Initial measurements of the coherent signal amplitude and phase are diffractionlimited (Marouf and Tyler, 1982). Measurement of the direct signal phase allows reconstruction of the observations to remove diffraction effects, yielding highresolution profiles of the complex ring transmittance, hence of optical depth and phase-shift profiles (Marouf et al., 1986). Achievable resolution is a small fraction of 
the corresponding Fresnel scale of diffraction and is determined by several factors, including geometry of the occultation orbit, stability of the reference phase, SNR of the observations, and processing complexity (Marouf et al., 1986). A better Cassini USO and an optimized set of Cassini occultation orbits promise significant improvements in achievable resolution over the Voyager observations.

The second component of the perturbed signal is the scattered or incoherent signal, a spectral-broadened component that represents the fraction of average power scattered by ring particles and intercepted by the ground receiving station (Marouf et al., 1982). The spectral broadening is caused by the Doppler shift introduced by the relative motion of the spacecraft and ring particles. Its time history (spectrogram) provides information regarding the relative abundance of meter size particles within broad ring features that can be resolved in the measured spectrograms.

The measured near-forward scattered signal of a resolved ring feature provides significant information about the distribution of particle sizes that populate the feature, its physical thickness, and the degree of particle crowding, among other physical parameters. Procedures have been developed to invert the measurements to recover physical model parameters for the classical many-particle-thick ring model (Marouf et al., 1982, 1983) and for the thin-layers ring model (Zebker et al., 1983, 1985). The more general problem of crowded and clustered ring models of arbitrary thickness is a subject of continued investigation (Marouf, 1994, 1996, 1997).

Contribution to the scattered signal measured during occultation is dominated by the diffraction lobes of large ring particles. Particles of radius a larger that the spacecraft antenna radius $(2 \mathrm{~m})$ have diffraction lobes of width narrower than the spacecraft antenna beamwidth, and information regarding their size distribution is captured in scattered signal measurements. The limit on the upper particle size captured is set by the sampling interval of the measured collective diffractionpattern, hence by the SNR of the measurement, and is typically few to several tens of meters. Thus, scattered signal measurement during occultation yields the particle size distribution over the range $2 \mathrm{~m}<a<$ several tens of meters. Other techniques are used to extend the distribution to the range $a<2 \mathrm{~m}$.

Over the millimeter to decimeter size range, measurement of the differential extinction and phase shift of the X-, S-, and Ka-bands coherent may be used to constrain the size distribution. In the case of Voyager, measured differential optical depth and phase shift of the coherent X- and S-band signals (3.6 and $13 \mathrm{~cm}-\lambda$ ) provided clear evidence for the presence of ring particles of sizes in the centimeter to decimeter radius range, both in local and global ring features (Tyler et al., 1983; Marouf and Tyler, 1985; Marouf et al., 1986). The additional availability of the Ka-band $(0.94 \mathrm{~cm}-\lambda)$ signal on Cassini will allow similar inference of the abundance of the population of millimeters to centimeters size ring particles.

Particles of size in the decimeter to meter size range do not differentially affect the coherent signals significantly and have diffraction lobes that are essentially isotropic over the spacecraft antenna beam width. Determination of their size distribution is based on a yet different technique, referred to here as the bistatic-scattering 
technique. It relies on observation of the scattered signal with the direction of the boresight of the spacecraft antenna shifted away from the earth direction so as to sample the broader diffraction lobes of particles in this range. Because the width of a diffraction lobe of a particle of diameter $D=2 a$ observed at wavelength $\lambda$ is roughly $\lambda / D$, the maximum angular shift of the spacecraft antenna boresight is determined by the minimum particle size whose diffraction lobe is to be sampled and is wavelength dependent.

Sampling the diffraction lobes of particles of radius $a \geq 20 \mathrm{~cm}$, for example, requires bistatic-scattering observations over a maximum angular range equal to approximately five times the antenna beamwidth at the wavelength of interest. Thus, for both X-and Ka-bands, maneuvers to shift the antenna boresight away from the earth direction are limited to no more than a few degrees change in the antenna boresight direction. The exact limit is set by available SNR. Optimal maneuver strategies to observe bistatic-scattering given available SNR are still under development.

The capability of determination of the particle size distribution of resolved ring features over the millimeters to tens of meters range (millimeter to decimeter using differential extinction, decimeters to meters using bistatic scattering, and meters to tens of meters using forward-scattering) is a unique capability of the radio science ring observations. For ring features for which bistatic scattering may be noise limited or not available, a selfconsistent model fitting approach that bridges the lower and upper ends of the distribution provides a viable alternative (Marouf $e t$ al., 1983; Zebker et al., 1985).

\subsubsection{Major Requirements}

The quality of ring occultation observations is determined by the phase and amplitude stability of the radio link, available SNR, and the geometry of occultation orbits, among other factors. These impose stringent requirements on the performance of the radio subsystems on board the spacecraft and at the ground receiving stations, as well as on the design of the Cassini tour.

Consider first the requirements on the phase stability of the radio link, a critical parameter for successful reconstruction of the diffraction-limited direct signal measurements. In the absence of phase noise, the limit on achievable radial resolution $\Delta R$ is set by the Fresnel scale of diffraction $F$ and the width $W$ of the radial interval over which diffraction limited measurements are processed, where $\Delta R=2 F^{2} / W$ (Marouf et al., 1986). In reality, finite phase instability over W limits actual achievable resolution to a larger value $\Delta R_{\phi}>\Delta R$, where $\Delta R_{\phi}$ is determined by the nature of the random reference phase fluctuations, usually dominated by the behavior of the USO onboard the spacecraft (Marouf et al., 1986).

In the Voyager case, phase stability of the USO (Allan deviation $\sigma_{y} \simeq 5 \times 10^{-12}$ at $1 \mathrm{~s} \mathrm{time} \mathrm{interval)} \mathrm{limited} \Delta R_{\phi}$ to the range $\Delta R_{\phi} \geq 250 \mathrm{~m}$ (Marouf et al., 1986). The Cassini USO is over ten times better than Voyager $\left(\sigma_{y} \simeq 2 \times 10^{-13}\right.$ at $\left.1 \mathrm{~s}\right)$. Calculations based on the assumptions of a random walk phase noise model (white 
frequency noise) and likely Cassini occultation orbits indicate that $\Delta R_{\phi} \geq 10 \mathrm{~m}$, more than an order of magnitude improvement over Voyager. A requirement that the Allan deviations of the atomic frequency standard used at the earth receiving stations be at least three times better than that of the USO ensures that the latter is the limiting factor. Actual Cassini resolution will depend, of course, on in-flight performance of the USO and ability to model and remove long term phase drift, and other factors including the degree of variability of $F$ over $W$ and available SNR (Marouf et al., 1986).

Like Voyager, Cassini conducts all occultations in a downlink mode (that is, transmission from the spacecraft and reception on the earth), hence the available free-space signal-to-noise ratio $\mathrm{SNR}_{0}$ is limited by the relatively small transmitted power. Indeed, both spacecraft have comparable $\mathrm{SNR}_{0}$ (for Cassini, $\mathrm{SNR}_{0} \simeq 52$, $40,41 \mathrm{~dB} / \mathrm{Hz}$ for the $\mathrm{X}-, \mathrm{S}-$, and Ka-band signals, respectively). Unlike Voyager, Cassini enjoys the advantage of arriving at Saturn when the ring system is almost fully open as seen from the earth (ring opening angle $B \simeq-24^{\circ}$ ), and remains in orbit as $B$ gradually increases to a Voyager-like value of about $-6^{\circ}$ over the four-year mission lifetime. Given a ring feature of normal optical depth $\tau$, the actual measurement SNR is reduced from its free-space value $\mathrm{SNR}_{0}$ by the factor $\exp [-\tau / \sin (B)]$ (due to attenuation by ring particles). For a $\tau=1$ ring feature, for example, the measurement SNR is a factor of 1200 higher at $B=24^{\circ}$ than at a Voyager-like $B=6^{\circ}$; the SNR is 1.5 million times higher for $\tau=2$ !

Given the extreme sensitivity of the measured SNR on the ring opening angle, a major requirement on the Cassini tour design was to implement a set of radio occultation orbits as early as possible after Saturn orbit insertion (SOI). The early occultations take full advantage of a unique opportunity provided by nature to probe all ring features, including the $\mathrm{B}$ Ring, with the limited available $\mathrm{SNR}_{0}$. An additional requirement of an occultation distance $D \simeq 4-6 R_{\mathrm{S}}$ ensures moderate Fresnel scale $F$, hence moderate complexity in reconstruction of the diffraction limited observations. A remarkable set of early near-diametric occultation orbits was designed and implemented by the Mission Design Team and is an integral part of the selected orbital tour. The orbits span the range $24^{\circ}<B<20^{\circ}$. Exceptional Xband radial resolution of $100 \mathrm{~m}$ or better is expected everywhere, including regions of Ring B of normal optical depth $\tau \leq 2.5$, an order of magnitude improvement over Voyager. Requirements for two additional occultation opportunities at intermediate $\left(B \simeq 15^{\circ}\right)$ and small $\left(B<10^{\circ}\right)$ ring opening angle complement the first set and allow more complete characterization of the variability of the observables with $B$, longitude, and time.

Additional requirements on occultation orbit geometry are necessary to improve the spatial resolution of scattered signal measurements. As indicated before, the intrinsic resolution is determined by the size of the spacecraft antenna "footprint" on the ring plane. The footprint can be very large (with a characteristic scale of thousands to hundreds of thousands of $\mathrm{km}$, depending on wavelength, the spacecraft distance $D$ behind the ring, and the ring opening angle $B$ ). The resolution may be 
improved if contours of constant Doppler shift over the footprint align closely with boundaries of ring features so that the contribution of a given feature may be identified in the measured spectrograms (Marouf et al., 1982). The Voyager flyby trajectory was optimized in part to enhance this alignment (Marouf et al., 1982), allowing spatial resolution in the range $1000-6000 \mathrm{~km}$ to be achieved (Marouf et al., 1983; Zebker et al., 1985). Similarly, Cassini early occultation orbits have been optimized to provide good Doppler contour alignment with ring boundaries. Spatial resolution as good as $500-1000 \mathrm{~km}$ is expected for some occultations.

\subsection{ATMOSPHERIC AND IONOSPHERIC OCCULTATION EXPERIMENTS}

The refraction of monochromatic radiation emitted from the spacecraft toward earth as it passes through a spherical or oblate atmosphere produces Doppler shifts in the received frequency that allow the retrieval of refractivity as a function of altitude. This in turn leads to vertical profiles of electron density in the ionosphere and of density, pressure, and temperature in the neutral atmosphere. Much of the value of occultation profiles lies in their high vertical resolution, typically better than 1 $\mathrm{km}$. This is to be contrasted with that achievable from passive remote sounders, for which the vertical resolution is more comparable to a scale height.

\subsubsection{Scientific Objectives}

2.5.1.1. Atmospheres.

a) Saturn: The Pioneer 11 spacecraft provided the first radio occultation of Saturn's ionosphere and atmosphere (Kliore et al., 1980a; Kliore and Patel, 1980), with ingress and egress soundings both at equatorial latitudes. Later, Voyager 1 and 2 gave two additional occultations, which sounded latitudes near the equator, at $36^{\circ} \mathrm{N}, 31^{\circ} \mathrm{S}$, and $73^{\circ} \mathrm{S}$ (Lindal et al., 1985; Tyler et al., 1981, 1982).

All the retrieved temperature profiles (Figure 1) in the neutral atmosphere have a well-defined troposphere (where temperature decreases with altitude), a tropopause, or temperature minimum, located at the $\approx 60-80 \mathrm{mbar}$ level, and a stratosphere (where temperature increases with latitude). The Voyager occultations, which had higher signal-to-noise ratio than those from Pioneer 11, gave atmospheric profiles that extended from a few tenths of 1 mbar to 1.3 bar. The lower pressure limit was dictated by the USO stability on the spacecraft, signal-to-noise ratio, and the resulting ability to separate the effects of the neutral atmosphere from the ionosphere on the refractivity. The limit at 1.3 bar resulted from absorption by $\mathrm{NH}_{3}$, which extinguished the signal transmitted from the spacecraft to earth.

Both the Pioneer and Voyager temperature profiles at low latitudes exhibit a marked undulatory structure in the upper troposphere and stratosphere that is suggestive of vertically propagating waves. Vertical wavelengths are $\sim 2$ scale heights, although smaller-scale behavior is also present. The undulations are more subdued at mid and high latitudes. This structure has not been interpreted or analyzed in 

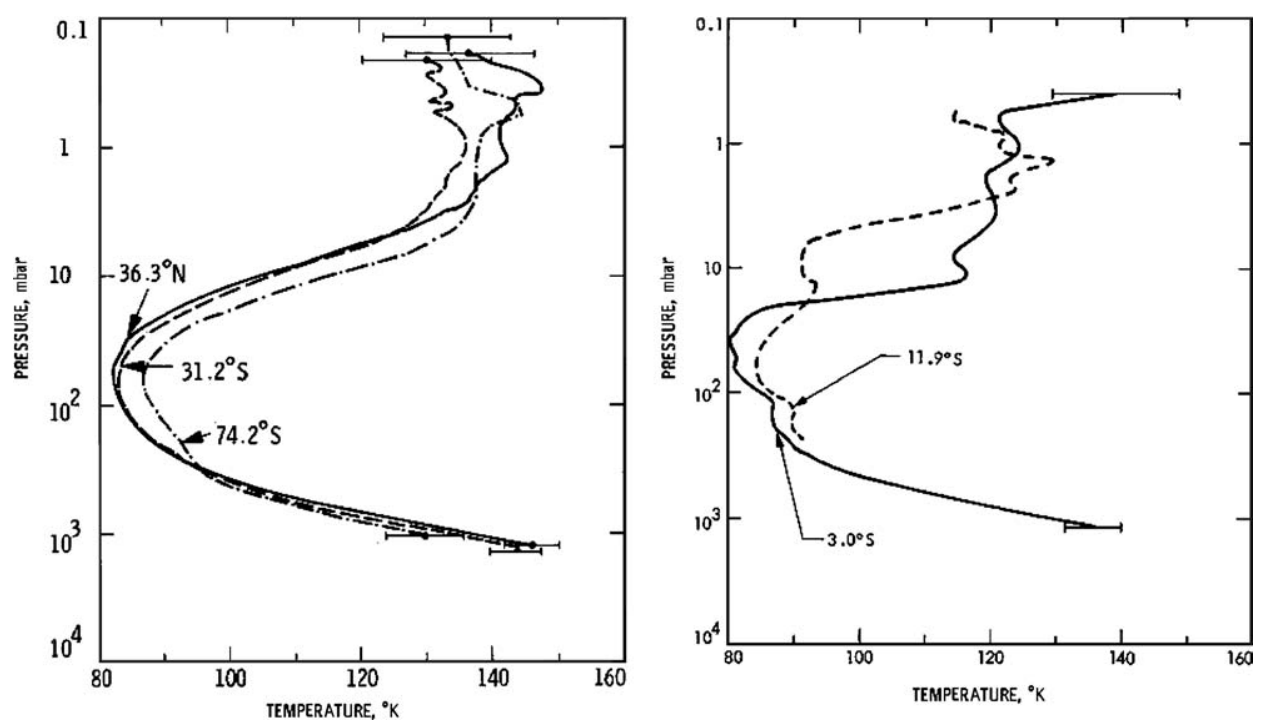

Figure 1. Temperature profiles in Saturn's atmosphere retrieved from Voyager and Pioneer 11. The dashed curve in the right panel is from the Pioneer egress (Kliore et al., 1980b). After Lindal et al. (1985).

any depth. In their analysis of the zonal (i.e., east-west) variations of temperatures retrieved from the Voyager infrared spectroscopy experiment (IRIS), Achterberg and Flasar (1996) found evidence of a coherent wave structure in the tropopause region that extended from $40^{\circ} \mathrm{N}$ to low latitudes. They deduced that it was most probably a Rossby wave, forced by a critical-layer instability at the more northern latitude. The wave amplitude they derived, $\pm 1 \mathrm{~K}$, seems consistent with the reduced amplitude seen in the mid-latitude radio-occultation profiles. Coverage of the equatorial region, particularly at southern latitudes, by IRIS, which was primarily a nadir-viewing instrument, was minimal, because of interference from Saturn's rings and, for Voyager 2, a malfunction of the remote-sensing scan platform. Temperatures retrieved from radio occultations show structure in Jupiter's atmosphere that is similar to Saturn's (Lindal et al., 1981). Allison (1990) has suggested that the structure in the equatorial profiles of Jupiter is produced by Rossby waves.

Waves are meteorologically significant, because they can be sensitive indicators of the conditions of the background mean atmosphere through which they propagate. In their study, Achterberg and Flasar (1996) were able to relate the observed meridional structure of the thermal wave they identified to the thermal structure of Saturn's atmosphere and spatial variation of its zonal winds. Waves can also be dynamically important, as they typically transport angular momentum over large distances. This could be an important factor in maintaining the large-scale zonal 
wind system on Saturn, which is notwell understood. Cassini is expected to provide occultations yielding temperature profiles at nearly 50 locations on Saturn, with a reasonably good distribution in latitude. This is far denser coverage than has been realized to date. With the zonal structure provided by the mapping capabilities of the composite infrared spectrometer (CIRS), wave structure in Saturn's troposphere and stratosphere should be well characterized.

Knowledge of temperature and pressure as functions of altitude and latitude leads to the zonal wind $u$ through the gradient wind equation,

$$
u \sin \Lambda\left(2 \Omega+\frac{u}{r \cos \Lambda_{p c}}\right)=-\frac{1}{\rho r}\left(\frac{\partial P}{\partial \Lambda}\right)_{\phi},
$$

and the thermal wind equation,

$$
\frac{\partial}{\partial \ln P}\left(u \sin \Lambda\left(2 \Omega+\frac{u}{r \cos \Lambda_{p c}}\right)\right)=\frac{R}{m r}\left(\frac{\partial T}{\partial \Lambda}\right)_{P} .
$$

Here $r$ is radius to the planet's center of mass, and $\Omega$ is the internal uniform planetary rotation rate of Saturn, presumably equal to that implied by the kilometric radiation modulation $\left(=1.66 \times 10^{-4} \mathrm{~s}^{-1}\right) ; P$ is pressure, $\rho$ is atmospheric mass density, $T$ is temperature, $R$ is the universal gas constant, and $m$ is the mean molecular weight of the atmosphere; $\Lambda$ is the planetographic latitude, determined by the intersection of the local normal to the equipotential surface, and $\Lambda_{p c}$ is planetocentric latitude, determined by the intersection of the line to the planet center of mass with the equatorial plane; $\phi$ denotes the geopotential,

$$
\phi=\int_{0}^{z} g d z^{\prime}
$$

where $g$ is the local effective gravitational acceleration, representing both gravitational attraction and the centrifugal repulsion associated with the uniform planetary rotation. The geopotential function $\phi$, therefore incorporates both gravity and uniform rotation; the altitude coordinate $z$ is normal to the geopotential surface. Note that in Equation (26) the gradient in pressure is at constant altitude relative to the geopotential associated with uniform rotation. In Equation (27) the gradient in temperature is along isobars. Because of Saturn's rapid planetary rotation, the geostrophic term, the one containing $2 \Omega$ on the left-hand sides of Equations (26) and (27), generally dominates.

The Pioneer 11 and Voyager soundings were too sparse for inferring winds, but the much denser coverage afforded by the Cassini radio occultations make this much more feasible. Although the occultations will not densely sample longitude, they will sample latitude very well. Temperatures retrieved from the Voyager IRIS spectra indicate that, atleast in the upper troposphere and lower stratosphere, the zonal variations of temperature are smaller than the variation with latitude. Thus the latitude variations in the pressure and temperature fields are fairly good indicators of the zonal winds on Saturn. Comparison with the temperatures retrieved from the 
CIRS instrument, which can obtain global maps, will facilitate this derivation. A very important advantage for radio science lies in the capability to retrieve pressure as a function of altitude, and hence obtain the zonal winds directly through the application of Equation (26) (c.f., Newman et al., 1984). When using temperatures to infer winds from Equation (27), as, for example CIRS must do, the winds need to be specified along a (usually lower) boundary. Winds will also be derived with high spatial resolution from cloud tracking using both the visible (ISS) and perhaps the near infrared (VIMS) images. In this analysis there always remains some ambiguity in the altitudes probed at a particular observing wavelength. Comparison of the winds derived from cloud tracking with those obtained from Equation (26) using radio science data should greatly facilitate the altitude assignment in the cloudtracking studies.

The sensitivity of the radio signal passing through Saturn's atmosphere to absorption by gaseous ammonia permits the retrieval of vertical profiles of $\mathrm{NH}_{3}$ below the 1-bar level. Lindal et al. (1985) have presented such profiles from the Voyager Saturn occultations, as well as from those for Jupiter (Lindal et al., 1981). The large number of Cassini occultations will permit a mapping of the three-dimensional distribution. Both passive ground-based microwave observations and observations at infrared and visible wavelengths have indicated that gaseous and condensed $\mathrm{NH}_{3}$ vary latitudinally over Saturn and Jupiter. These variations are probably indicative of the vertical motion field near the 1-bar level. Particularly intriguing in the observed radiances at radio and far-infrared wavelengths is a "warm" broad band on Saturn that is centered about $30^{\circ} \mathrm{N}$ (Conrath and Pirraglia, 1983; Grossman et al., 1990). The corresponding latitude range in the southern hemisphere is much colder. This has been interpreted as caused by a diminution in cloud opacity and ammonia gas in the northern latitude range. Curiously, the Voyager radio occultation data at $36^{\circ} \mathrm{N}$ and $31^{\circ} \mathrm{S}$ give no hint of such asymmetry in the derived $\mathrm{NH}_{3}$ abundance (Lindal et al., 1985). Whether an "atypical" region at the northern latitude was sounded by the radio occultations is open to question. The more complete coverage of Cassini should help address questions like this.

The helium abundance of the giant planets is an important indicator of their formation and evolution. One of the more sensitive methods to determine the helium abundances of the atmospheres of the outer planets from remote sensing has entailed the combination of radio-occultation soundings with far infrared spectra. The radio occultations provide profiles of the refractivity versus altitude, from which $T(z)$ is derived. For uniform composition, the quantity $T(z) / m$ is invariant. To first order, varying the assumed composition shifts the profile $T(z)$ towards higher or lower values without changing its shape. If the source of infrared opacity of the atmosphere is known, a synthetic infrared spectrum can be computed and compared to an observed spectrum.

Comparison of Voyager radio occultation retrievals with IRIS spectra led to values of helium that were slightly depleted relative to solar abundance on Jupiter (Gautier et al., 1981) and quite depleted on Saturn (Conrath et al., 1984). This 
appeared to be consistent with the notion that differentiation of the heavier helium from hydrogen was occurring in the planets' interiors.

Recently, however, direct determinations of the helium abundance from the Galileo probe (Niemann et al., 1996; von Zahn and Hunten, 1996) have yielded values that are higher than that determined from Voyager and more nearly solar. Recent planetary evolution models (Hubbard et al., 1999) have also called into question the low value of helium derived for Saturn, and they suggest that the current value of Saturn's intrinsic luminosity, which derives from cooling of its interior, is more consistent with a larger helium abundance for Saturn's atmosphere than was derived from Voyager data. Conrath and Gautier (2000) have also re-examined the helium retrieval from Voyager IRIS observations of Saturn. In this analysis, they used the redundant information in the IRIS spectrum between 200 and $600 \mathrm{~cm}^{-1}$ to simultaneously retrieve profiles of temperature, the parafraction of hydrogen, and the helium abundance. In this spectral region the principal opacity is from absorption by the pressure-induced dipole of molecular hydrogen, caused by collisions with other hydrogen molecules and with helium. The absorption is sensitive to all three of the aforementioned atmospheric variables. Although this "internal" method of determining the helium abundance is not as sensitive as combining radio-occultation profiles with infrared spectra, Conrath and Gautier (2000) nonetheless were able to conclude the helium abundance must be higher than the value derived earlier.

Evidently there is some as yet unidentified source of systematic error in the earlier determination. Having CIRS spectra at the numerous locations of Cassini radio occultations, where the haze structure varies, may help elucidate the source of the problem and produce a more reliable determination of the helium abundance. There are no planned in situ measurements of helium on Saturn in the foreseeable future, so a reliable determination via remote sensing remains a high priority.

b) Titan: Prior to the arrival of the Voyager 1 spacecraft at Saturn, the surface pressure and temperature of Titan's atmosphere were not well determined. Indeed, predictions of the surface pressure differed by a factor of 100 (Danielson et al., 1973; Caldwell, 1977; Hunten, 1978). In traversing the Saturn system, Voyager 1 flew behind Titan and provided a diametric occultation near the equator as viewed by Earth. Because of the gross uncertainty in the atmosphere base pressure, one could not perform a normal limbtracking maneuver (see Section 2.5.2.2), in which the spacecraft attitude is continually adjusted to ensure that the refracted radio waves reach Earth. This would have required a reasonably accurate model of the mean vertical structure of Titan's atmosphere for predicting the attitude control. Instead, the Voyager team hedged its bets, using constant offsets of the spacecraft antenna boresight from the line of sight to Earth, $0.1^{\circ}$ during the ingress, and $2.4^{\circ}$ during the egress (Lindal et al., 1983; Hinson et al., 1983). It turns out that the bending angle in the neutral atmosphere increases more or less monotonically with depth, reaching $2^{\circ}$ for the ray that grazes the surface. The Voyager spacecraft antenna transmitted at both S- and X-bands. The S-band data, for which the half-power full width of 
the antenna was $2.5^{\circ}$, covered the full range of atmospheric pressures during both ingress and egress, whereas the $\mathrm{X}$-band data, with an antenna width of $0.6^{\circ}$, only proved useful in limited altitude ranges, above $28 \mathrm{~km}$ during ingress and within a few $\mathrm{km}$ of the surface during egress.

The Voyager 1 soundings provided vertical profiles of temperature and pressure at altitudes below $200 \mathrm{~km}$. As discussed in Section 2.5.2, the refractivity profile is the fundamental quantity retrieved from the soundings, and obtaining temperature and pressure profiles requires that the atmospheric bulk composition be specified. The combination of data from the thermal infrared spectrometer (IRIS) with the radio occultation data led to the conclusion that the mean molecular weight of Titan's atmosphere is $\sim 28 \mathrm{AMU}$, highly suggestive of $\mathrm{N}_{2}$ as the dominant constituent (Tyler et al., 1981; Hanel et al., 1981; Vervack et al., 1999). This was supported by the identification of several nitriles in the IRIS spectra, as well as the detection of atomic and molecular nitrogen in the thermosphere by the ultraviolet spectrometer on Voyager (Broadfoot et al., 1981). Lindal et al. (1983) retrieved vertical profiles of temperature and pressure from the Voyager soundings, assuming an atmosphere of pure $\mathrm{N}_{2}$. The ingress and egress soundings were nearly identical in the troposphere, and they obtained a surface temperature and pressure of $94 \mathrm{~K}$ and $1.5 \mathrm{bar}$, respectively. Below $4 \mathrm{~km}$ the lapse rate in temperature was nearly dry adiabatic, suggesting efficient mixing by small-scale convection.

The effect of composition on the retrieved temperature profiles is an important consideration for Titan. When the composition is spatially uniform, the retrieved temperature, as a function of altitude, scales linearly with the molecular mass (see Equation (54)). However, when there is a spatially varying condensible, such as $\mathrm{CH}_{4}$, contributing to the refractivity, the behavior of temperature with altitude can be markedly different. Flasar (1983) retrieved temperatures for atmospheres with different $\mathrm{N}_{2}-\mathrm{CH}_{4}$ mixtures. He demonstrated that a vertical profile of saturated $\mathrm{CH}_{4}$ implied increasing lapse rates of temperature near the surface. By imposing the stability constraint that the lapse rate not exceed the dry adiabat, he deduced that the lowest $4 \mathrm{~km}$ of Titan's atmosphere had to be unsaturated and the maximum $\mathrm{CH}_{4}$ mole fraction just above the surface was $\sim 0.09$ or less. Later, Lellouch et al. (1989) did a systematic study of $\mathrm{N}_{2}-\mathrm{CH}_{4}{ }^{-36,38} \mathrm{Ar}$ atmospheres. Retrieving temperature profiles from the radio occultation refractivity profiles, they found a range of possible surface temperatures, from 92.5 to $101 \mathrm{~K}$, and tropopause temperatures in the range $70.5-74.5 \mathrm{~K}$. All the profiles were qualitatively similar in shape to that for the $\mathrm{N}_{2}$ atmosphere. Although Ar was initially thought to be a bulk constituent of Titan's atmosphere (see e.g., Samuelson et al., 1981), no evidence of it has turned up, and indeed, recent work (Strobel et al., 1993; Courtin et al., 1995; Samuelson et al., 1997) suggests that the Ar mole fraction may be quite low, $<0.01$. For atmospheres composed of $\mathrm{N}_{2}$ and $\mathrm{CH}_{4}$, Lellouch et al. (1989) found the range of allowable temperatures to be smaller: maximum temperatures are $71.8 \mathrm{~K}$ at the tropopause and $95.2 \mathrm{~K}$ just above the surface; the minimum possible temperatures are unchanged from before. Although the implied range of uncertainty amounts 
to only $\sim 3 \mathrm{~K}$, the IRIS spectra indicate that this is comparable to the meridional contrast seen at the surface and in the troposphere (Flasar et al., 1981). Because of the likely spatial variation of tropospheric $\mathrm{CH} 4$, it is important to reduce the current uncertainty in the radio occultation results, which are mostly attributable to uncertainty in the composition.

Despite this limitation, the Voyager radio-occultation profiles have formed the basis of much of the analysis of Titan's atmospheric structure, for example, in constructing global radiative-equilibrium and radiative-convective models (Samuelson, 1983; McKay et al., 1989). The occultation profiles have also been directly used in the interpretation of IRIS spectra. Radiances in the thermal infrared depend both on atmospheric temperature and the infrared opacity. In the absence of independent information, the separation of these two effects requires redundancy in the infrared spectrum. This was generally not the case for the IRIS spectra, because much of the thermal infrared spectrum accessible to IRIS was dominated by aerosol opacity, whose heterogeneous distribution is not known (see Flasar (1998b) for a discussion). Hence, it was not possible to retrieve atmospheric temperatures from the spectra over most altitudes. Temperatures could only be retrieved in the upper stratosphere, using the $v_{4}$-band of $\mathrm{CH}_{4}$ as a "thermometer", under the assumption of a uniform abundance of CH4 in the stratosphere (Flasar, 1998a). Some information on physical temperatures was also available at the tropopause and at the surface (Flasar et al., 1981). The latter was possible because of a "window" that occurs in the thermal infrared spectrum near $530 \mathrm{~cm}^{-1}$. Although surface emission is an important component of the radiances here, the stratospheric emission is not negligible.

Several studies of the spatial distribution of stratospheric compounds, based on emission lines in the mid-infrared portion of the IRIS spectra, have relied on the radio occultation refractivity profiles. For instance, Coustenis and Bézard (1995), in retrieving the meridional distribution of several hydrocarbons and nitriles, started with a mean temperature profile based on the equatorial radio-occultation profiles. They assumed that temperatures in the troposphere did not vary meridionally, but rescaled temperatures in the stratosphere to vary linearly with altitude from the radio-occultation profiles at the 40-mbar level, just above the tropopause, to the temperatures retrieved from the IRIS spectra in the upper stratosphere. Similarly, Courtin et al. (1995) and Samuelson et al. (1997) compared synthetic spectra, computed from temperature profiles derived from the radio occultation refractivities, with IRIS spectra in the far-infrared $\left(200-600 \mathrm{~cm}^{-1}\right)$ to constrain the distribution of tropospheric $\mathrm{CH}_{4}$. They both reached the amazing result that, in a global sense, $\mathrm{CH}_{4}$ in the middle and upper troposphere must be supersaturated to provide the opacity needed to yield a good match between the synthetic and observed spectra. Samuelson et al. (1997) went on to derive the meridional distribution of tropospheric $\mathrm{CH}_{4}$, including that near the surface. To do this, they had to account somehow for temperature variations in the troposphere. In effect, they used the IRIS spectra to constrain the temperatures at the tropopause and at the surface, and assumed that 
occultation profiles retained their general shape with latitude. Although this is not unreasonable, it still remains an educated guess.

The Voyager 1 radio occultation data provided one of the few indications of waves in Titan's atmosphere. Hinson et al. (1983) analyzed the intensity scintillations found in the occultation profiles. They found a region of weak scintillation extending from 25 to $90 \mathrm{~km}$ altitude that they attributed to a vertically propagating internal gravity wave with little or no attenuation. At $44 \mathrm{~km}$, near the tropopause, the implied vertical and horizontal wavelengths were $1 \mathrm{~km}$ and $4 \mathrm{~km}$, respectively, and the wave amplitude was $\sim 1 \mathrm{~K}$. They also detected strong scintillations over the altitude range $5-10 \mathrm{~km}$. Later simulations by Friedson (1994) suggested that these might be attributed to an internal gravity wave that saturates and breaks near 15 km (see Flasar (1998b) for a more detailed review). Hinson and Magalhães (1991) have discussed how an atmospheric wave modulates a radio wave as it traverses an occulting atmosphere and is refracted. The amplitude response of the signal is dominated by diffraction effects, and it is most sensitive to atmospheric structure on scales that are smaller than the diameter of the first Fresnel zone, $2 \sqrt{\lambda D}$, where $\lambda$ is the wavelength of the radio wave, and $D$ the distance of the spacecraft to the occulting limb. The contribution from larger-scale fluctuations is filtered out. For the Voyager occultation, $2 \sqrt{\lambda D} \approx 1-3 \mathrm{~km}$ at the $\mathrm{S}$ - and $\mathrm{X}$-band wavelengths, and it is not surprising that Hinson et al. (1983) detected waves with a vertical scale that was comparable to this. The phase response of the received signal, on the other hand, is dominated by atmospheric structure with vertical scales that are large compared to the Fresnel scale, for which diffraction effects are small. The temperature profiles of Titan's atmosphere retrieved from the Voyager occultations do suggest some larger-scale wave-like structure (Lindal et al., 1983), but the characterization of this is incomplete. While systematic analyses of the phase scintillations have been performed on radio occultation soundings of some of the outer planets (Hinson and Magalhães, 1991, 1993), such a study has yet to be undertaken for the Titan data.

The Cassini radio science experiment is more capable than Voyager's, and it has several attractive features relevant to the issues discussed. One of the most important is that the orbiter will provide a distribution of Titan occultations with latitude, so that temperature profiles can be retrieved from measurements instead of from inspired guesswork. The Cassini thermal infrared spectrometer, CIRS, is also much improved from its predecessor, IRIS. It extends to longer wavelengths through the submillimeter portion of the spectrum, which will permit the pressure-induced $S(0)$ line of $\mathrm{N}_{2}$ to be used as another "thermometer," probing the upper troposphere and middle and lower stratosphere (Flaser et al., 2004). However, except for the surface, CIRS cannot retrieve temperatures below $30 \mathrm{~km}$ altitude. The radio occultation soundings provide the only means of doing so from the orbiter. By virtue of its limb sounding capability, CIRS can use the $530 \mathrm{~cm}^{-1}$ radiances observed on the limb, for which deep space is the background, to subtract the stratospheric contribution to the radiances observed in nadir viewing, so that the surface emission and temperature can be better determined. The combination of this with radio occultation refractivity 
profiles will permit a more accurate determination of the distribution of $\mathrm{CH}_{4}$ in the lower troposphere.

The latitude coverage will also provide important information on the zonal wind field, through application of Equations (26) and (27) to the retrieved pressures and temperatures, as discussed earlier for Saturn. With only two equatorial occultations, this was not possible with the Voyager data. The ability to assign a reference altitude scale to the zonal wind velocities may be even more important for Titan than for Saturn, as the identification and tracking of discrete features by the ISS and VIMS experiments to determine horizontal wind velocities will probably be more difficult for the former body.

Figure 2 depicts the characteristic vertical resolution of the occultations over the altitude range in which temperature and pressure profiles will be retrieved. Because the USO on Cassini is a factor of 20 more accurate than the one used by Voyager, atmospheric refraction should be detectable at higher altitudes, and temperature and pressure profiles up to $300 \mathrm{~km}$ altitude should be achievable. In the stratosphere, the vertical resolution is $2 \sqrt{\lambda D}$. The vertical resolution is higher in the troposphere, because differential refraction and defocusing become important, and the Fresnel zone flattens vertically (Haugstad, 1978; Karayel and Hinson, 1997). Figure 2 indicates that vertical resolution lies between $\sim 1 \mathrm{~km}$ and $100 \mathrm{~m}$, depending on the altitude and wavelength used, with Ka-band soundings providing the higher resolution. As discussed earlier, in Section 2.4, higher resolution is possible through inverse Fresnel reconstruction techniques. Although planetary atmospheres present

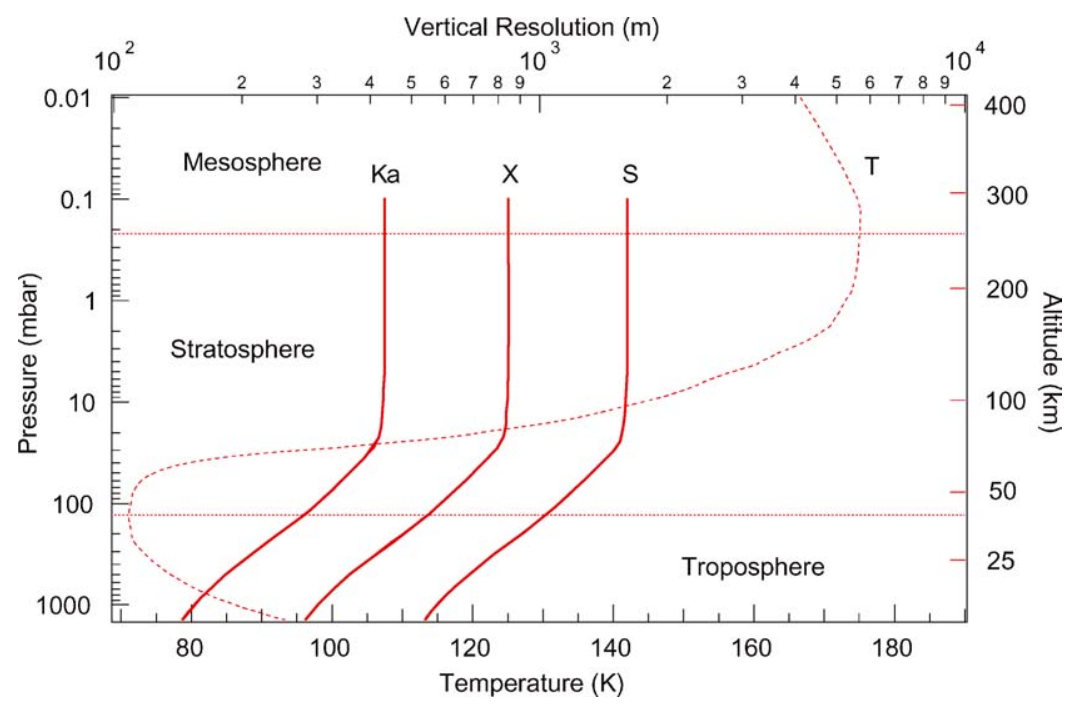

Figure 2. Vertical resolution (solid curves) of temperature profiles on Titan retrieved from radio occultations at S-, X-, and Ka-bands, for a nominal spacecraft-to-occulting limb distance of $5000 \mathrm{~km}$. Dashed curve: temperature retrieved from Voyager radio occultation soundings below $200 \mathrm{~km}$, after Lellouch et al. (1989); above $200 \mathrm{~km}$, temperatures are from Yelle's (1991) model. 
a more complex situation than rings, which behave as sharp edges in a vacuum, recent work on sub-Fresnel-scale inversion for atmospheres has been encouraging (Karayel and Hinson, 1997).

The high vertical resolution afforded by radio science is important for elucidating several atmospheric phenomena. In addition to resolving a large spectrum of vertically propagating waves, already discussed, the occultation soundings should be able to detect any thin cloud or aerosol layers in the stratosphere or troposphere, provided they have sufficient opacity to modify the temperature profile. The structure of the convectively mixed planetary boundary layer is of interest in understanding the exchange of heat and volatiles between the atmosphere and surface. The Voyager occultations indicate that the mixed layer extends to $\sim 4 \mathrm{~km}$ altitude at the equator, but its altitude probably varies with latitude. On Earth, the structure of the planetary boundary layer is complex, reflecting both dry and moist convective processes. Often the upper boundary is marked by a sharp stable inversion as, for example, at the top of the trade cumulus layer at subtropical latitudes (see e.g., Augstein, 1978). The latitude variation of the the top of the planetary boundary layer can be indicative of meridional circulations in the troposphere, with subsiding regions characterized by lower heights of the boundary layer. Aside from the Huygens atmosphere structure investigation on the probe, which will take data at only one location, radio occultations will provide the highest resolution of tropospheric vertical structure.

The addition of a new wavelength [Ka band, $0.94 \mathrm{~cm}$ ] should prove very important for atmospheric studies. In addition to providing the highest vertical resolution, it may prove to be an important probe of atmospheric absorption. Voyager occultations gave no indication of atmospheric absorption at S- and X-bands (Lindal et al., 1983), but the absorption properties of Titan'satmosphere in the Ka-band are not known.

\subsubsection{Ionospheres.}

a) Introduction: Any object in our solar system that has a neutral gas envelope surrounding it, due either to gravitational attraction (e.g., planets) or some other processes such as sublimation (e.g., comets), has an ionosphere. Radio occultation observations during the flybys of the Pioneer 11 (Saturn) and Voyager 1 and 2 spacecraft have clearly established the existence of a robust ionosphere around Saturn.

The retrieved electron density profiles exhibit primary maxima at altitudes 2000$3000 \mathrm{~km}$ above the 1-bar level. Those from Pioneer, in particular, exhibit a complex structure that suggest multiple layers of ionization. Multiple-layer structure in the Voyager ionospheric profiles is much more subdued, but this may be in part due to the fact that they were inverted only for higher altitudes. Originally, these profiles were retrieved only for $1500 \mathrm{~km}$ or higher (one profile from Voyager 2 did extend down to $700 \mathrm{~km}$ ) (Eshleman et al., 1979a,b). Recently, Hinson et al. (1998a) have extended the electron density retrievals from the Voyager 2 occultations by Jupiter down to $300 \mathrm{~km}$, and at the lower altitudes layered structure is quite in evidence. 
The Pioneer measurements were carried out using a single frequency S-band signal, the Voyager measurements employed dual frequencies at $\mathrm{S}$ and X-bands. The signal-to-noise ratios achieved in the Voyager observations were significantly better than the Pioneer ones. Furthermore the dual frequency technique is particularly advantageous in the signal analysis/inversion process in the presence of sharp ionospheric layers, which may lead to multipath propagation.

The three-frequency Cassini radio science subsystem will provide opportunities to obtain a significant number of new and high quality electron density profiles with a good coverage of latitudes and local time. These density profiles, combined with information on the time variation of the peak electron density values, to be deduced from the observations of Saturn electrostatic discharges (SED's) by the plasma radio wave experiment, will help to advance our understanding of the physical and chemical processes controlling the behavior Saturn's ionosphere. Furthermore, ionospheric information, combined with upper atmospheric data from the UV occultation experiment will also help to elucidate the aeronomy of Saturn's upper atmosphere.

The radio occultation data from the flyby of Titan by the Voyager 1 spacecraft provided an estimate of the peak electron density at a solar zenith angle near the terminator. Cassini will provide, during its baseline mission, further occultation opportunities, with improved signal-to-noise ratios. The information from these occultations, combined with in situ measurements of the ionospheric plasma, will lead to major advances in describing Titan's aeronomy.

b) Saturn: As indicated above our presently available direct information regarding the ionosphere of Saturn is based on a handful of electron density profiles obtained by radio occultation measurements and peak electron density values deduced from SED's. This information, combined with neutral atmospheric data obtained mainly from UV occultation measurements, has been used develop our present understanding of Saturn's ionosphere. The major neutral constituent in Saturn's upper atmosphere is molecular hydrogen, therefore the major primary ion, which is formed by either photoionization or particle impact, is $\mathrm{H}_{2}^{+}$. In the equatorial and low-latitude regions electron-ion pair production is believed to be mainly due to solar EUV radiation, while at higher latitudes impact ionization by precipitating particles becomes very important. Although over $90 \%$ of all initial ions produced are $\mathrm{H}_{2}^{+}$, their actual concentration is very small, because they undergo rapid charge transfer reactions. The rest of the discussion in this section will be based, for the sake of brevity, on photoionization (photodissociation) only, because particle ionization leads to similar products and processes. Solar radiation with wavelength short of 2768,804 , and $686{ }^{\circ}$ A,respectively, leads to

$$
\begin{aligned}
& \mathrm{H}_{2}+h v \rightarrow \mathrm{H}+\mathrm{H}, \\
& \mathrm{H}_{2}+h v \rightarrow \mathrm{H}_{2}^{+}+e, \\
& \mathrm{H}_{2}+h v \rightarrow \mathrm{H}^{+}+\mathrm{H}+e .
\end{aligned}
$$


The resulting neutral atomic hydrogen can also be ionized

$\mathrm{H}+h v \rightarrow \mathrm{H}^{+}+e$.

At high altitudes where hydrogen atoms are the dominant neutral gas species, $\mathrm{H}^{+}$can only recombine directly via radiative recombination, which is a very slow process, given the recombination rate of order $\sim 10^{-12} \mathrm{~cm}^{-3} \mathrm{~s}^{-1}$. It was suggested some time ago that $\mathrm{H}^{+}$could charge-exchange with $\mathrm{H}_{2}$ excited to a vibrational state $v>4$. The vibrational distribution of $\mathrm{H}_{2}$ is not known, but recent calculations (Cravens, 1987) appear to indicate that, while there are some vibrationally excited molecules present at Saturn, the corresponding charge exchange rate is not significant. $\mathrm{H}_{2}$ is very rapidly transformed to $\mathrm{H}_{3}^{+}$, especially at the lower altitudes where $\mathrm{H}_{2}$ is dominant; $\mathrm{H}_{3}^{+}$then in turn undergoes dissociative recombination

$$
\begin{aligned}
\mathrm{H}_{2}^{+}+\mathrm{H}_{2} & \rightarrow \mathrm{H}_{3}^{+}+\mathrm{H}, \\
\mathrm{H}_{3}^{+}+e & \rightarrow \mathrm{H}_{2}+\mathrm{H} .
\end{aligned}
$$

Significant uncertainties have been associated with the dissociative recombination rate of $\mathrm{H}_{3}^{+}$. However, recent measurements have shown that the rate is rapid, even if the ion is in its lowest vibrational state (Sundstrom et al., 1994). Models based upon the above discussed processes predict an ionosphere which is predominantly $\mathrm{H}^{+}$, because of its long lifetime $\left(\sim 10^{6} \mathrm{~s}\right)$. In these models $\mathrm{H}^{+}$is removed by downward diffusion to the vicinity of the homopause $(\sim 1,100 \mathrm{~km})$, where it undergoes charge exchange with heavier gases, mostly hydrocarbons such as methane, which in turn are lost rapidly via dissociative recombination. The main difficulties with these models are:

1. the calculated ionospheric density at the apparent main peak is about an order of magnitude larger than the observed one;

2. the altitude of the calculated ionospheric main peak is much lower than the observed one; and,

3. the predicted long lifetime of $\mathrm{H}^{+}$is inconsistent with the observed large diurnal variations in the electron density peak.

A number of suggestions have been put forward during the last decade in order to overcome these difficulties. The most recent and successful models are based on the suggestion/assumption that water from the rings is being transported into Saturn's upper atmosphere, which then modifies the chemistry of the ionosphere. The presence of $\mathrm{H}_{2} \mathrm{O}$ results in the following catalytic process

$$
\begin{aligned}
\mathrm{H}^{+}+\mathrm{H}_{2} \mathrm{O} & \rightarrow \mathrm{H}_{2} \mathrm{O}^{+}+\mathrm{H}, \\
\mathrm{H}_{2} \mathrm{O}^{+}+\mathrm{H}_{2} \mathrm{O} & \rightarrow \mathrm{H}_{3} \mathrm{O}^{+}+\mathrm{OH}, \\
\mathrm{H}_{3} \mathrm{O}^{+}+e & \rightarrow \mathrm{H}_{2} \mathrm{O}+\mathrm{H} .
\end{aligned}
$$

A block diagram of the chemistry scheme involving water is shown in Figure 3. The models which take into account this water chemistry and the recent values of 


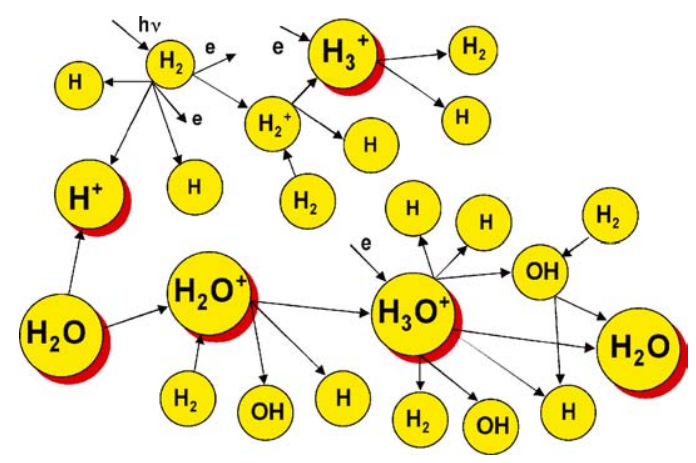

Figure 3. Block diagram of the chemistry scheme, including water (from Schunk and Nagy, 2000).

SATURN IONOSPHERE

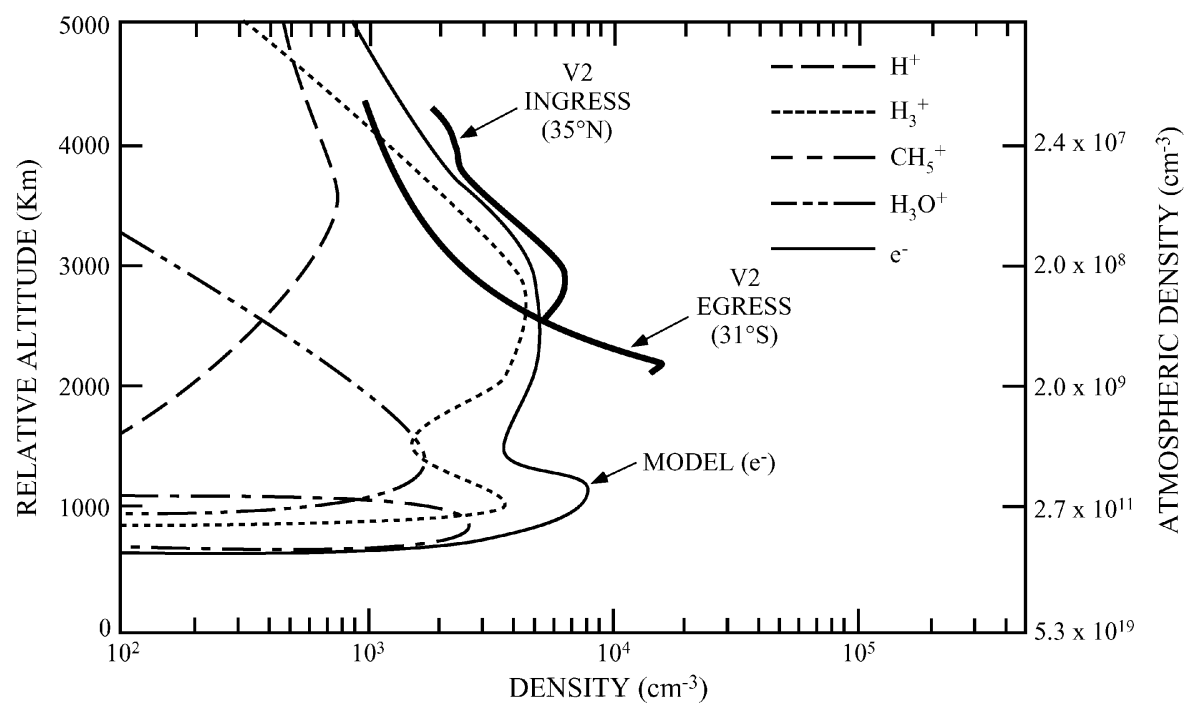

Figure 4. Calculated and measured (solid lines) ion densities for the ionosphere of Saturn. The calculations take into account the inux of water and a soft electron precipitation source (from Waite and Cravens, 1987).

the recombination rate for $\mathrm{H}_{3}^{+}$, lead to ionospheres consisting mainly of $\mathrm{H}^{+}, \mathrm{H}_{3}^{+}$ and $\mathrm{H}_{3} \mathrm{O}^{+}$. It was shown that a downward flux of water from the rings into the atmosphere of the order of $1-10 \times 10^{7} \mathrm{~cm}^{-2} \mathrm{~s}^{-1}$ leads to electron density values consistent with the observations (see Figure 4); however, no current model has been able to reproduce the implied large diurnal variations (Majeed and McConnell, 1996).

c) Titan: Titan is surrounded by a substantial atmosphere and therefore one expects a correspondingly significant ionosphere. The only opportunity for a radio 
occultation measurement of such an ionosphere occurred when Voyager 1 was occulted by Titan. A careful analysis of those data by Bird et al. (1997) found that the peak electron density is about $2.4 \times 10^{3} \mathrm{~cm}^{-3}$.

Indirect evidence of the existence of an ionosphere was also obtained by the low-energy plasma analyzer measurements in the plasma wake. The various ionization sources which may be responsible for the formation of Titan's ionosphere are solar Extreme Ultra-Violet (EUV) radiation, photoelectrons produced by this radiation, and magnetospheric electrons. Cosmic rays can cause some low altitude ionization (e.g., Capone et al., 1976) and proton and other ion precipitation may also make some contributions. To complicate matters even further, it is possible that under certain circumstances, e.g., high solar wind pressure, Titan will be beyond the magnetopause and in the magnetosheath of Saturn. Under these circumstances, the nature and intensity of the particle impact ionization source will be quite different. Calculations to date have concentrated on EUV and magnetospheric electron impact ionization, the two sources believed to be the dominant ones.

The next question which needs to be addressed is how do magnetospheric electrons reach the upper atmosphere. Gan et al. (1992) considered draped magnetic field lines and assumed that electrons enter these field lines far down the wake region at the "end" of these field tubes, for ram conditions, but can enter in a radial fashion on the wakeside. Keller et al. (1992) found that, contrary to earlier suggestions, photoionization is the main source for the dayside ionosphere, followed by photoelectron impact, and finally magnetospheric electron sources. Of course, magnetospheric electrons must dominate in the nightside ionosphere. The comprehensive photochemical calculations of Keller et al. (1992), found that the electron density peak is about $5 \times 10^{3} \mathrm{~cm}^{-3}$ at an altitude around $1,100 \mathrm{~km}$, for $\chi=60^{\circ}$, and that the peak density is $3 \times 10^{3} \mathrm{~cm}^{-3}$, at an altitude of about $1,195 \mathrm{~km}$, for $\chi=90^{\circ}$, close to the Voyager results. Similar results were obtained by Ip (1990), using his model published a few years earlier. Both Ip (1990) and Keller et al. (1992) predicted that $\mathrm{HCNH}^{+}$is the major ion near the density peak. The block diagram shown in Figure 5 indicates the production and loss pathways leading to $\mathrm{HCNH}^{+}$. Fox and Yelle (1997) and Keller et al. (1998) have published results from their new Titan ionospheric models. The Fox and Yelle (1997) results indicate that the pseudo-ion $\mathrm{C}_{x} \mathrm{H}_{y}^{+}$(this is the sum of all ions with three or more carbon atoms) is the major one. Their result is shown in Figure 6. The Keller et al. (1998) model gives very similar electron densities and peak altitude. However, they still predict that $\mathrm{HCNH}^{+}$is the major ion in a narrow region near the peak; the sum of the heavy hydrocarbon ions is also very significant in their model.

A variety of different studies examined the issue of the transition from chemical to diffusive control in the ionosphere. Simple time constant considerations, as well as more detailed model solutions, have indicated that the transition from chemical to diffusive control takes place in the altitude region around $1,500 \mathrm{~km}$. The magnetospheric plasma velocity $\left(\sim 120 \mathrm{~km} \mathrm{~s}^{-1}\right)$ is subsonic $\left(\sim 210 \mathrm{~km} \mathrm{~s}^{-1}\right)$ and 


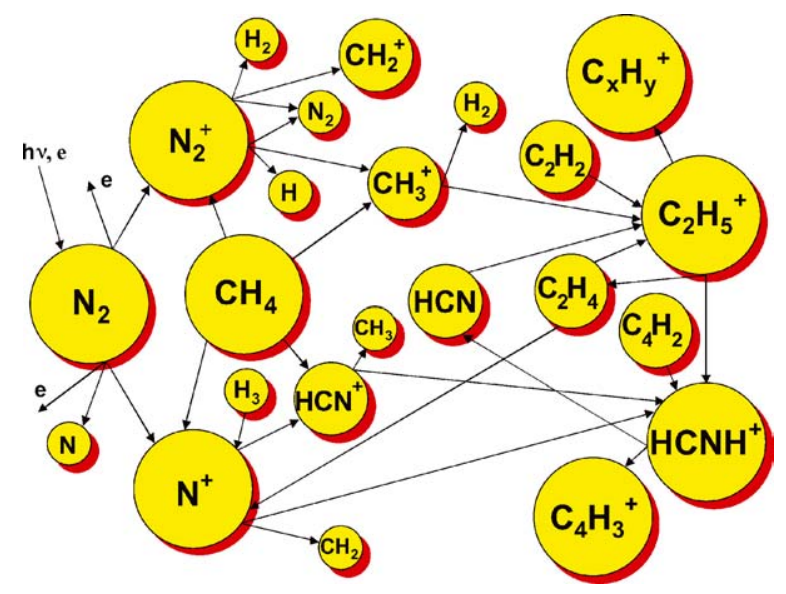

Figure 5. Block diagram of a proposed ion chemistry scheme for Titan (from Schunk and Nagy, 2000).

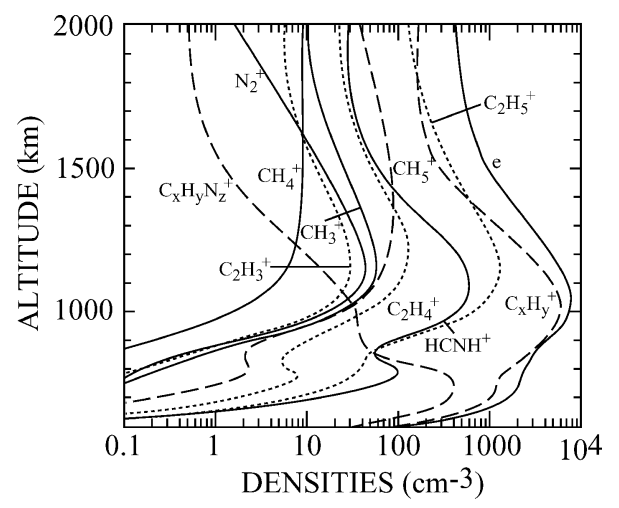

Figure 6. Calculated electron density profiles for Titan, assuming particle impact ionization by 30 and $50 \mathrm{eV}$ electrons (from Fox and Yelle, 1997).

superalfvenic $\left(\sim 64 \mathrm{~km} \mathrm{~s}^{-1}\right)$, therefore, no bow shock is formed and the magnetic field is gradually slowed as it enters Titan's exosphere by mass loading. The magnetic field strength increases, piles up and eventually drapes around Titan. This piled up magnetic field, similar to the so-called magnetic barrier at Venus, is expected to be the dominant source of pressure against the ionosphere. Using reasonable magnetospheric parameters Keller et al. (1994) found that the total incident magnetospheric pressure is about $1.6 \times 10^{-9}$ dynes $\mathrm{cm}^{-2}$. Using a peak electron density of $5 \times 10^{3} \mathrm{~cm}^{-3}$, this means that if the corresponding plasma temperature is greater than $700 \mathrm{~K}$, the ionosphere is capable of holding off the external plasma. This pressure corresponds to about $20 \mathrm{nT}$, much of which is convected into the upper ram ionosphere, resulting in near-horizontal magnetic fields. Figure 7 is a pictorial 


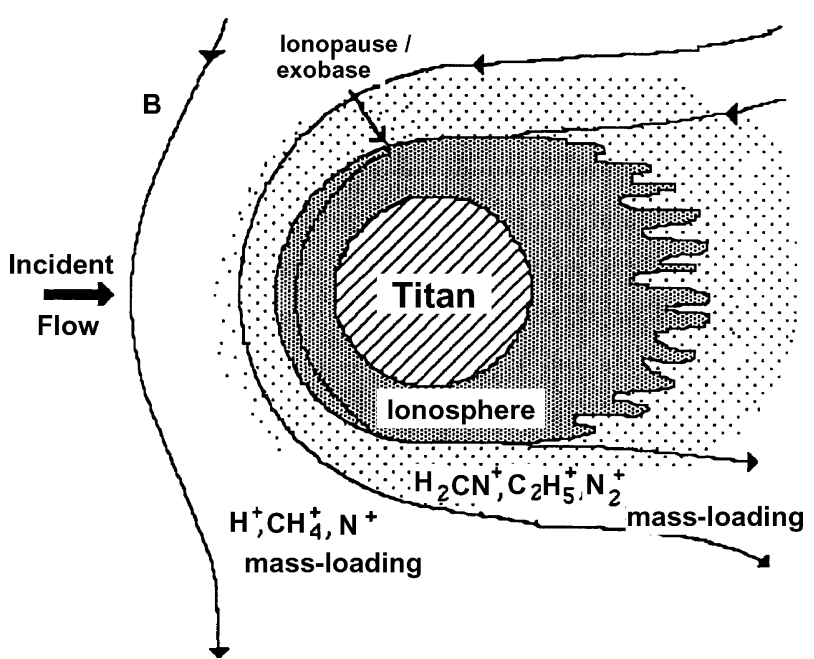

Figure 7. Pictorial representation of the interaction of Titan's ionosphere with magnetospheric plasma on the ram side (from Keller et al., 1994).

representation of the situation for the ramside ionosphere. As indicated earlier, there are a large number of cases that can be considered, such as full solar and magnetospheric ionization sources, near-terminator conditions, and only magnetospheric sources.

d) Icy satellites: The environments of the icy satellites of Jupiter, Europa, Ganymede, and Callisto, have been observed through radio occultations of the Galileo spacecraft. Europa, and probably Callisto, were found to have a very tenuous ionosphere (Kliore et al., 1997) most likely produced by the sputtering effects of Jupiter's magnetospheric particles upon their icy surfaces (Johnson et al., 1998). There are no deliberate occultations of Cassini by any of Saturn's icy satellites designed into the current satellite tour.

\subsubsection{Techniques}

2.5.2.1. The Radio Occultation Technique. Radio occultation, in which a spacecraft emitting one or more coherent monochromatic radio signals, appears to move behind a planet or satellite as seen from the earth, affords an opportunity for the spacecraft-to-earth radio links to traverse the ionosphere and atmosphere of the occulting body. The interpretation of the observed effects of refraction by the planetary atmosphere and ionosphere allows one to determine the vertical electron density structure in the ionosphere and the temperature-pressure profiles and absorption characteristics of the neutral atmosphere (c.f., Fjeldbo, 1964; Kliore et al., 1964).

This technique has been applied successfully to measure the characteristics of the ionospheres and atmospheres of Mars (Fjeldbo et al., 1968; Kliore et al., 1965; 
Kliore et al., 1969; Kliore et al., 1972a; Kolosov et al., 1975; Lindal et al., 1979), Venus (Fjeldbo et al., 1971; Howard et al., 1974b; Kliore et al., 1967; Kolosov et al., 1976; Kliore et al., 1979; Kliore and Patel, 1980a, 1982; Savich et al., 1986; Jenkins et al., 1993; Kliore and Mullen, 1989; Kliore and Luhmann, 1991; Yakovlev et al., 1991), Mercury (Fjeldbo et al., 1976a; Howard et al., 1974a), Jupiter (Kliore et al., 1974; Fjeldbo et al., 1975; Fjeldbo et al., 1976b; Kliore et al., 1976, 1977; Eshleman et al., 1979a,b; Hinson et al., 1997; Lindal et al., 1980, 1981; Flasar et al., 1998), Saturn (Kliore et al., 1980b; Lindal et al., 1985; Tyler et al., 1981), Uranus (Lindal et al., 1987; Tyler et al., 1986), Neptune and Triton (Tyler et al., 1989; Lindal, 1992), Titan (Lindal et al., 1983), Saturn's rings (Marouf and Tyler, 1985), and Jupiter's satellites Io, Europa, Ganymede, and Callisto (Kliore et al., 1975; Hinson et al., 1998a, in press; Kliore et al., 1997; Kliore et al., 1998a, b). The radio occultation technique depends on being able to invert the observed changes in the frequency and amplitude of the radio signals during the time of the occultation to produce vertical profiles of the index of refraction and absorption coefficient. For the case of spherical symmetry, in which the atmospheric structure depends only on the radial distance from the center of the planet, the inversion techniques have been firmly established (c.f., Fjeldbo and Eshleman, 1968; Kliore, 1972b). This technique also provides a good approximation in the case of oblate planets, in which case the center of refraction is determined by the local radius of curvature at the occultation location (c.f., Kliore et al., 1976, 1977). The method by means of which which the refraction angle, or bending angle, $\varepsilon$, and the ray asymptote distance, $p$, are determined for each time $t_{0}$ can be deduced with reference to Figure 8.

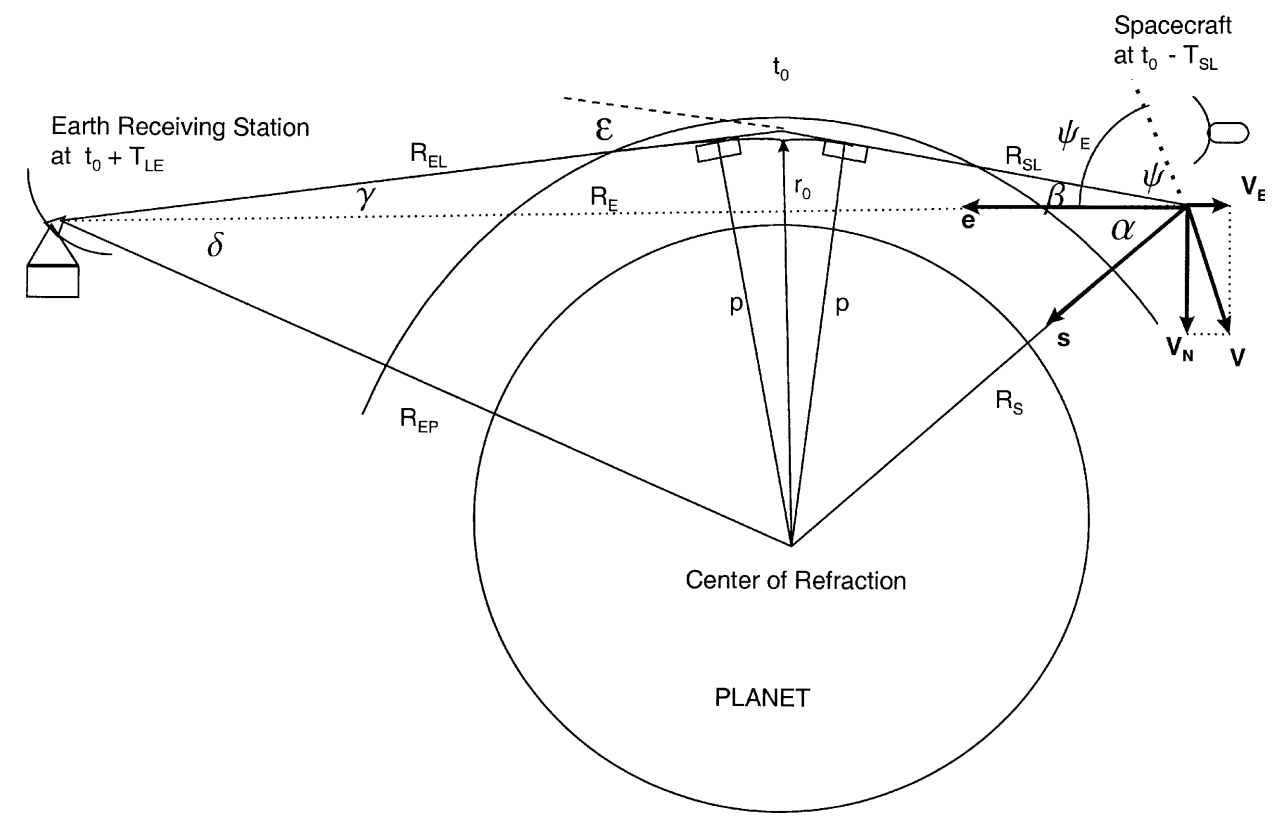

Figure 8 . Geometry of a radio occultation observation. 
Figure 8 displays the geometry in a plane defined by the position of the center of refraction at time $t_{0}$ (the time at which the photon in question came closest to the center of refraction), the position of the spacecraft at time $t_{0}-T_{\mathrm{SL}}$ (the time when the photon was emitted from the spacecraft antenna), and the position of the receiving antenna on earth at time $t_{0}+T_{\mathrm{LE}}$ (the time when the photon reached the receiving station, i.e., the time of the observation). These times and positions are iteratively computed from the ephemerides of the spacecraft, the planet center, and the earth center relative to the Sun, as well as the exact location of the receiving station. This defines the plane of refraction at $t_{0}$. Referring to Figure 8, the Doppler frequency observed with no refraction is

$$
v_{\mathrm{E}}=\frac{\nu_{0}}{c} v_{\mathrm{E}},
$$

where $v_{\mathrm{E}}$ is $\mathbf{v} \cdot \hat{\mathbf{e}}$, the component of the planetocentric inertial spacecraft velocity $\mathbf{v}$ in the plane of refraction, in the direction of the virtual earth (receiving station at time of observation). If $\delta v$ is defined as the frequency residual, relative to the "free space" Doppler frequency, that is caused by a refractive bending angle $\varepsilon$, then it may be shown that (c.f., Phinney and Anderson, 1968)

$$
\cos \left(\psi_{\mathrm{E}}-\beta\right)=\frac{\nu_{\mathrm{E}}+\delta v}{\nu_{\mathrm{S}}},
$$

where

$$
v_{\mathrm{S}}=\frac{v_{0}}{c} v,
$$

and,

$$
\psi_{\mathrm{E}}=\arccos \left(\frac{v_{\mathrm{E}}}{v}\right) .
$$

Equation (39) can then be used to determine the angle $\beta$, which under the usual condition of $R_{\mathrm{EL}} \gg R_{\mathrm{SL}}$ is equal to the bending angle, $\varepsilon$. The corresponding ray asymptote distance (ray parameter), $p$, can then be computed as

$$
p=R_{\mathrm{S}} \sin (\alpha+\beta),
$$

where

$$
\cos \alpha=\hat{\mathbf{e}} \cdot \hat{\mathbf{s}},
$$

and $\hat{\mathbf{s}}$ is the unit vector from the spacecraft position to the center of refraction. The actual bending angle is then

$$
\varepsilon=\beta+\arcsin \left(\frac{R_{\mathrm{SL}}}{R_{\mathrm{EL}}} \sin \beta\right)
$$

Thus, for each time of observation $t_{0 i}$ one can determine the corresponding bending angle and ray parameter, $\left(\varepsilon_{i}, p_{i}\right)$. In order to invert these data to obtain a vertical profile of the index of refraction, it is necessary to make use of the Abel 
integral transform (c.f., Fjeldbo and Eshleman, 1968; Fjeldbo et al., 1971; Kliore, 1972b). In a spherically stratified medium, the refractive bending angle can be expressed as

$$
\varepsilon(p)=-2 p \int_{p}^{\infty} \frac{d n}{d \chi} \frac{d \chi}{n \sqrt{\chi^{2}-p^{2}}} .
$$

where $n$ is the index of refraction. The inverse transform is then

$$
\ln n(p)=\frac{1}{\pi} \int_{p}^{\infty} \frac{\varepsilon(\xi) d \xi}{\sqrt{\xi^{2}-p^{2}}},
$$

and the radius of closest approach of the ray, $r_{0}$, corresponding to $n\left(r_{0}\right)$ is

$$
r_{0}=\frac{p}{n(p)} \text {. }
$$

The index of refraction in an atmosphere is very nearly 1.0, therefore it is more convenient to work with the refractivity, which is defined as

$$
N=(n-1) \times 10^{6} .
$$

In order to calculate profiles of pressure and temperature from the refractivity, it is necessary to define the chemical composition of the atmosphere. For the outer planets, the neutral atmosphere can be assumed to consist of hydrogen and helium. Under those conditions, the mass density, $\rho$, in $\mathrm{g} \mathrm{cm}^{-3}$ is related to the refractivity by

$$
\rho=\frac{\bar{m} N}{R q},
$$

where $\bar{m}$ is the mean molecular weight

$$
\bar{m}=f_{\mathrm{H}_{2}} m_{\mathrm{H}_{2}}+\left(1-f_{\mathrm{H}_{2}}\right) m_{\mathrm{He}} ;
$$

and $q$ is the specific refractivity

$$
q=0.26943\left(135.77 f_{\mathrm{H}_{2}}+34.51\left(1-f_{\mathrm{H}_{2}}\right)\right) .
$$

In the above equations, $f_{\mathrm{H}_{2}}$ is the number fraction of hydrogen in the atmosphere, the numerical refractivities of hydrogen and helium in Equation (51) are from Newell and Baird (1965), and $R$ is the universal gas constant. Assuming that the atmosphere is in hydrostatic equilibrium

$$
d P=g(z) \rho(z) d z
$$

and hence

$$
P(r)=P_{0}+\int_{r}^{r_{0}} P(z) g(z) d z,
$$


and the temperature is computed from the perfect gas law, as follows:

$$
T(r)=T_{0} \frac{N\left(r_{0}\right)}{N(r)}+\frac{\bar{m}}{R N(r)} \int_{r}^{r_{0}} N(z) g(z) d z .
$$

Thus, once one has a profile of the index of refraction in an atmosphere, and its chemical composition, any other geophysical property of the atmosphere can be readily computed. In case of the ionosphere, since the index of refraction is dependent only on the number of free electrons, it is independent of the ion composition of the ionosphere, and so the refractivity is directly related to the electron density, as follows:

$$
n_{\mathrm{e}}(r)=2.479 \times 10^{-14} N(r) v_{0}^{2},
$$

where $v_{0}$ is the transmitted frequency in $\mathrm{Hz}, N(r)$ is the refractivity in n-units, and $n_{\mathrm{e}}(r)$ is the electron density in $\mathrm{cm}^{-3}$.

The effects of absorption and scattering in a planetary atmosphere can be described by the absorptivity (or, absorption coefficient), $(\sigma)(r)$, having units of $\mathrm{dB} \mathrm{km}^{-1}$, as follows:

$$
\tau(r)=2 \int_{r}^{\infty} \sigma d s
$$

In Equation (56), $\tau$ represents the total absorptive attenuation, or extinction along the path of the ray, in $\mathrm{dB}$ as follows:

$$
10 \log \left(\frac{I}{I_{0}}\right)=-\tau
$$

where $I$ is the intensity of the signal. For a refracted ray tangentially propagating through a spherically stratified atmosphere with index of refraction $n(r)$, Equation (56) becomes

$$
\tau(p)=2 \int_{p}^{\infty} \frac{\sigma(\chi)\left(\frac{d r(\chi)}{d p}\right) \chi d \chi}{\sqrt{\chi^{2}-p^{2}}},
$$

and this can be inverted by using the Abel integral transform pair, c.f., (Jenkins et al., 1993) to yield the absorptivity

$$
\sigma(p)=-\frac{1}{\pi P}\left(\frac{d p(r)}{d r}\right) \frac{d}{d p} \int_{p}^{\infty} \frac{\tau(\xi) \xi d \xi}{\sqrt{\xi^{2}-p^{2}}} .
$$

It is customary to express the attenuation of a radio frequency signal in decibels relative to an unattenuated signal power level, $W_{0}$

$$
A(z)=10 \log \frac{W_{0}}{W(z)}
$$

The attenuation due to absorption is obtained by subtracting refractive defocusing attenuation and correcting for antenna mispointing

$$
\tau(z)=A(z)-A_{\text {def }}(z)+A_{\text {corr }}(z)
$$


where

$$
A_{\text {def }}(z)=10 \log \left(1-D(z) \frac{d \varepsilon(z)}{d z}\right),
$$

and

$$
D(z)=R_{\mathrm{S}}(z) \cos (\alpha+\beta)+p(z) \tan \frac{\varepsilon(z)}{2} .
$$

The value of the optical depth, $\tau(z)$ is then

$$
\tau(z)=A_{a}(z) \frac{\ln 10}{10} \simeq 0.23026 A_{a} .
$$

When a profile of the absorptivity, $\sigma(z)$ is obtained through the procedure outlined above, it can be used to infer the abundances of specific absorbers in the atmosphere, such as $\mathrm{NH}_{3}$ and $\mathrm{PH}_{3}$ in the atmosphere of Saturn, and the three frequencies available on Cassini will make it possible to determine their relative abundances. Radio occultation data from oblate or otherwise not spherically symmetrical bodies can also be inverted by applying raytracing techniques (Lindal, 1992; Melbourne et al., 1994)

2.5.2.2. Limb Tracking Maneuvers. During an occultation experiment, monochromatic waves at $\mathrm{X}, \mathrm{S}$, and/or Ka bands are downlinked to earth by the spacecraft high-gain antenna, using the onboard ultrastable oscillator to maintain coherence. When a planetary body with an atmosphere begins to occult the spacecraft, the radio wave transmitted from the spacecraft to earth traverses the atmosphere and is refracted. In a neutral atmosphere, the molecular constituents raise the index of refraction above one, and the wave is refracted toward higher atmospheric densities, more or less inward along the potential gradient. In an ionosphere, the electrons dominate refraction, and decrease the index of refraction to less than one. In this case the wave is refracted away from high electron densities. In general, the ionospheric electron density does not increase monotonically with depth, and there can be isolated peaks in electron density. The refraction of radio waves away from these peaks can cause complicated diffraction patterns, leading to so-called multipath effects with different frequencies being detected simultaneously at earth (see e.g., Fjeldbo et al., 1971; Hinson et al., 1997).

The effects of the atmosphere on the index of refraction are small. In the neutral portion of the atmosphere, these are largest at the highest barometric pressures traversed by the radio waves. At the surface of Titan $(\approx 1.5$ bar $)$ or at the $1-2$ bar level on Saturn, where $\mathrm{NH}_{3}$ will likely extinguish the received signal, the index of refraction deviates from 1 by only $10^{-3}$. In ionospheres, the differences are even smaller, typically $10^{-6}$ at S-band for an electron densities of $1 \times 10^{5} \mathrm{~cm}^{-3}$, less at $\mathrm{X}$ and $\mathrm{Ka}$ wavelengths. (The change to the index of refraction induced by electrons is proportional to the square of the wavelength in the radio spectrum; in a neutral atmosphere, the change is independent of wavelength.) However, these 


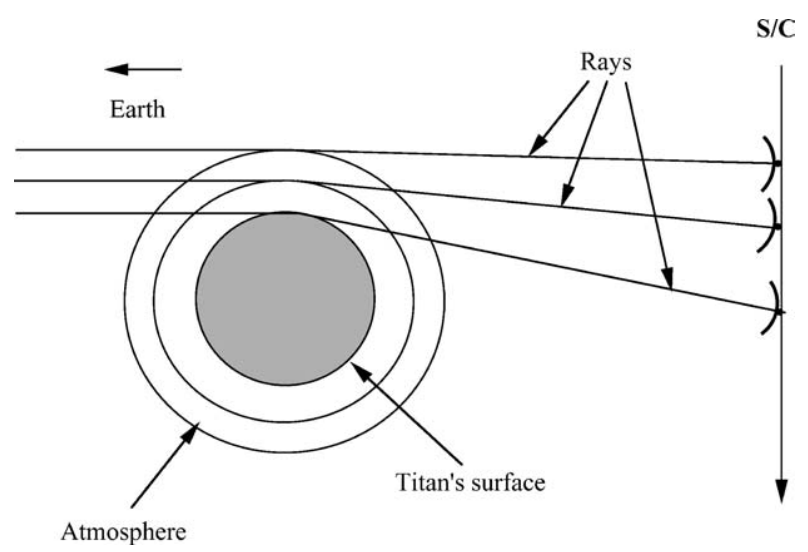

Figure 9. Idealized geometry for that portion of the entry phase of an occultation when the radio waves traverse the neutral portion of an atmosphere.

small differences, particularly in the neutral atmosphere, can induce large enough changes in the direction of the propagation of the radio wave and in its frequency in the earth-fixed frame of reference, relative to what would occur in the absence of the atmosphere, that an active strategy is required to avoid loss of the received signal.

Figure 9 illustrates an idealized geometry for that portion of the entry phase of an occultation when the radio waves traverse the neutral portion of an atmosphere. Here, the spacecraft moves on a trajectory perpendicular to the earth-spacecraft line of sight, but this does not materially affect the following discussion. During entry the radio waves, depicted as rays in the figure, progressively traverse deeper layers of the atmosphere, and the bending angle, $\varepsilon$, increases. At the surface of Titan, the bending angle amounts to $2^{\circ} \approx 34 \mathrm{mrad}$, and this is also comparable to that at the 2-bar level in Saturn's atmosphere. This angle is close to the full-width at half power of the S-band diffraction limited spacecraft high-gain antenna pattern, but much larger than those at X-band ( $9 \mathrm{mrad})$ and Ka-band $(2 \mathrm{mrad}$, designed to be slightly defocussed from the diffraction limit).

Hence the spacecraft high-gain antenna must continuously track the virtual image of earth on the occulting limb in order to avoid loss of the signal. This is necessary not only to maintain lock in the Doppler tracking, but also to measure the signal intensity accurately. The observed attenuation is the sum of that from refractive defocusing and that from absorption by the atmosphere. The former results from the rays being refracted through larger angles as they successively lie deeper in the atmosphere (Figure 9). This can be computed once the Doppler shifts in frequencies from refraction in the atmosphere are determined. The absorption is the difference between the observed attenuation and that computed from defocusing. It is important to distinguish between variations in signal intensity from atmospheric effects and those associated with errors in maintaining the virtual image of earth in the boresight of the antenna beam. Inevitably errors in both spacecraft 
pointing control (currently $2 \mathrm{mrad}$ ) and reconstruction (much smaller) arise during an occultation, and these need to be minimized.

The effects of refraction must also be taken into account when selecting the frequencies from the local oscillator to mix with the received signal at the DSN tracking station and the bandwidth to be used for the digitized recordings. The mixing must be done in order to convert the $\mathrm{GHz}$ frequencies of the received signal to the $\mathrm{kHz}$, or audio-range, so that digitization of the signal is possible. From Figure 9, the Doppler shift in frequency from refraction is given approximately as:

$$
\delta v=v_{0} \frac{v}{c} \varepsilon
$$

for small $\varepsilon$ to first order in $v / c$, where $v$ denotes the planetocentric spacecraft velocity component normal to the earth direction in the plane of refraction. For typical Titan occultations, this velocity is at most $5.6 \mathrm{~km} \mathrm{~s}^{-1}$. With $\varepsilon \approx 34 \mathrm{mrad}$, $\Delta v / v \sim 6 \times 10^{-7}$, or $\Delta v \sim 1.5 \mathrm{kHz}$ at S-band.

Generating the local oscillator frequency record for the receiving station thus requires a model atmosphere for the occulting body to simulate the effects of refraction on the frequency shift, so as to maintain the mixed signal near the center of the audio-range recording bandwidth. The bandwidth itself is selected to account for navigation errors and actual variations in atmospheric properties from the model. Typically, this is chosen as conservatively as possible to minimize the possibility of signal loss without making the digitized recordings intractable. For example, Galileo radio occultations by Jupiter and its satellites were recorded with a $2.5 \mathrm{-kHz}$ bandwidth at S-band (see e.g., Hinson et al., 1997; Kliore et al., 1997). Voyager occultations of the outer planets and Titan were recorded at larger bandwidths, as these atmospheres were less well characterized at the time: $5-25 \mathrm{kHz}$ was typical at S-band, with the exception of $50 \mathrm{kHz}$ for Saturn, because of the wider bandwidth required for the adjacent occultation of its rings (Lindal et al., 1981, 1983, 1985a, b, 1987, Lindal, 1992).

\subsubsection{Major Requirements}

As in the case of ring occultations, the quality of atmospheric and ionospheric occultation data depends on the phase and amplitude stability of the three radio links, the signal-to-noise ratio available on these links, and the orbital geometry which defines the occultation locations on the target body and their characteristics. Since atmospheric and ionospheric occultations will be conducted only in the oneway mode, the discussion of the USO stability and the SNR in Section 2.4.3 applies equally here. The requirements on the orbital geometry are given below.

The requirements for Saturn occultations are as follows:

1. A sufficient number of Saturn occultations well spaced in latitude in both the Northern and the Southern hemispheres. This is needed to provide data on zonal wind speeds at different altitudes and latitudes, as well as to sample the ionosphere structure and the ammonia abundance at different latitudes. 
The requirements for Titan occultations are:

1. A sufficient number of Titan occultations well spaced in latitude in both hemispheres to provide data for the derivation of the global zonal circulation of Titan's atmosphere.

2. An adequate sampling of the ionosphere at different locations relative to the magnetospheric ram direction.

3. Occultations that are not fast enough to require the use of thrusters to carry out the limb-tracking maneuvers (Section 2.5.2.2).

\section{Radio Science Instrument}

\subsection{OVERVIEW}

Among the Cassini instruments, the radio science instrument is unique in that is not confined to the spacecraft, and that part which is on the spacecraft is distributed among several subsystems. It can be regarded as a solar-system-sized instrument observing at microwave frequencies, with one end of the radio path on the spacecraft and the other end at DSN stations on the ground.

\subsubsection{Functional Description}

Figure 10 shows a functional block diagram abstracting those elements of the Cassini orbiter and the DSN that make up the radio science instrument. The instrument operates in two fundamental ways, distinguished by whether the microwave optical path has one or two legs.

For two-way measurements, the uplink can be a single carrier signal at either X-band $(\sim 7.2 \mathrm{GHz})$ or Ka-band $(\sim 34 \mathrm{GHz})$, or both carriers can be transmitted at the same time. These signals are generated by the DSN receiver/exciter system, using as a reference the local frequency and timing system. The DSN frequency standard is a combination of a Hydrogen Maser and a Sapphire Cavity Oscillator. The uplink signals are amplified, radiated through feed horns, and collimated by a large parabolic ground antenna, which is continuously aimed at the Cassini spacecraft. The actual transmission frequencies can be adjusted to allow the spacecraft receivers to lock to the uplink signals and to compensate, finite steps, for the main part of the Doppler effect between the earth and the Cassini Orbiter. In this mode spacecraft radio equipment forms a repeater, collecting the carrier signal with the spacecraft's high gain antenna (HGA), transforming it to one or more downlink frequencies $(2.3 \mathrm{GHz}, 8.4 \mathrm{GHz}$, or $32 \mathrm{GHz})$ that are coherent with the uplink, amplifying and re-collimating it, and sending it back to earth. The returning signal is detected using DSN ground receiving equipment, amplified and downconverted, and recorded for later analysis. In a variant of the two-way mode, the downlink is received at a different ground station; this three-way technique has been found very 


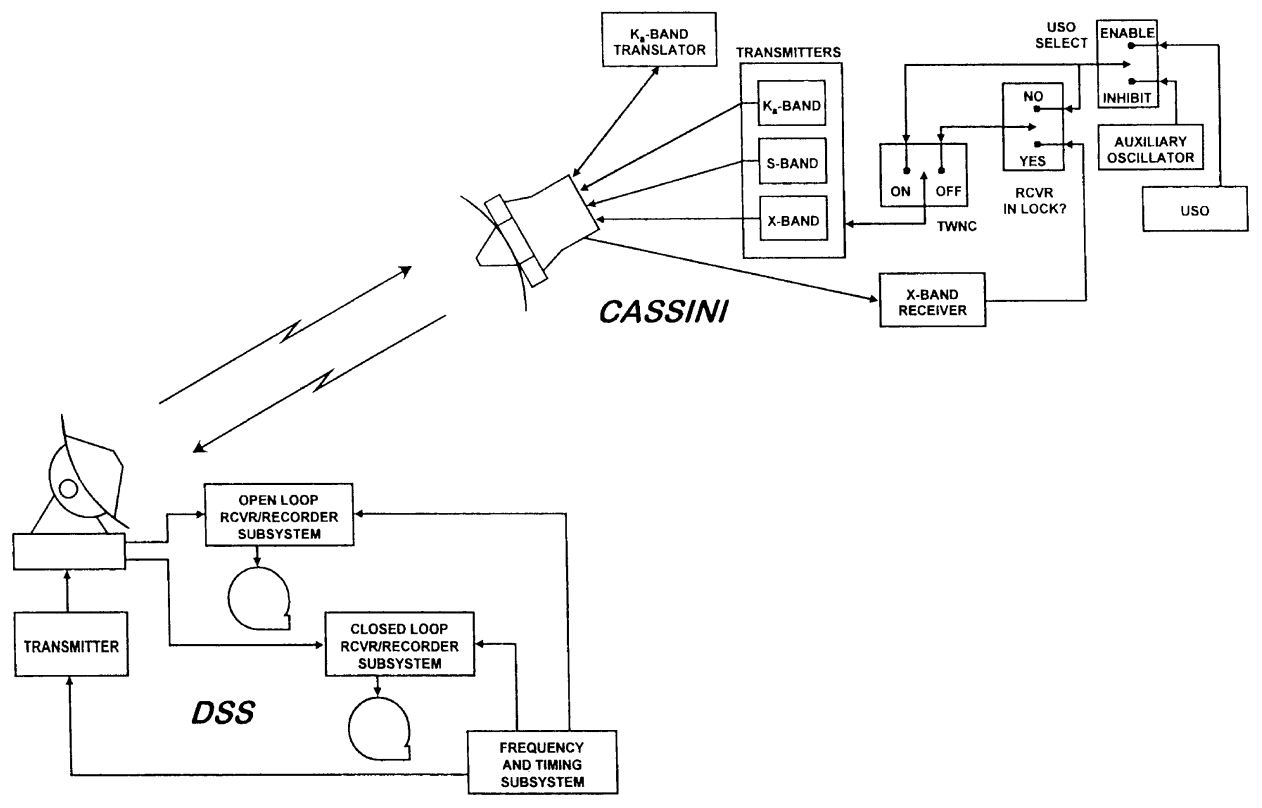

Figure 10. The distributed radio science instrument.

useful to isolate anomalous noise sources and to improve the science return from most of the radio science experiments.

For one-way measurements, the signal source is on board the Cassini orbiter. The output from on-board ultrastable oscillator (USO) is transformed to downlinks at S-band $(2.3 \mathrm{GHz}), \mathrm{X}$-band $(8.4 \mathrm{GHz})$, or Ka-band $(32 \mathrm{GHz})$ by elements in the radio frequency subsystem (RFS) and radio frequency instrument subsystem (RFIS). These signals are amplified and radiated through the HGA toward earth. After passing through the medium of interest, the perturbed signal is collected by a DSN antenna, amplified and downconverted, and recorded for later analysis.

\subsubsection{Measurements}

The scientific observables are phase, frequency, and amplitude perturbations on the $\mathrm{S}-, \mathrm{X}-$, and Ka-band signals that are transmitted between the spacecraft and the ground. These perturbations are induced by:

- passage through atmospheres, ionospheres, plasmas, and populations of particles (rings);

- gravitational accelerations of the spacecraft caused by nearby masses;

- relativistic effects.

Because the perturbations induced by the phenomena under investigation are very small, it is essential that sources of obscuring noise be minimized. The 
contribution of the Doppler effect due to the spacecraft and ground antenna motions must be removed, which is a key step in the processing of the data. In the spacecraft and ground equipment, this means that all components have to preserve as much dimensional and electrical stability as can be accomplished within mass, power, and environmental constraints. The key characteristics of the radio frequency (RF) signals have to be maintained over short and long integration times. Short-term stability is generally measured as phase noise. Stability over longer integration times is described by the so-called Allan deviation parameter $\sigma_{y}$, a statistical measure of contiguous differences of frequency measurements. In general, the requirements are that phase noise be $\geq 60 \mathrm{~dB}$ below the carrier in the frequency bands where radio science measurements are made, and that the Allan deviation range from $2 \times 10^{-13}$ at 1 -second integration time to $1 \times 10^{-15}$ at 1000 -second integration time.

Investigations dealing with the composition, chemistry, and dynamics of planetary atmospheres and ionospheres use the instrument in its one-way mode at all three downlink frequencies. Ring studies and measurement of general relativistic time delay during solar conjunction are also conducted using the noncoherent mode. Data samples for these experiments are integrated over short times $(\leq 1-100 \mathrm{~s})$, where the USO-driven signal provides the best stability. In the one-way mode, measurements can be started immediately after the spacecraft emerges from behind a planet or satellite. Using the one-way mode avoids potential lock up problems associated with a deep egress atmospheric occultation. The one-way path also avoids complications that would be introduced into data analysis by two passages, separated in time and space, through the target under study.

Two-way Doppler experiments in cruise and during the tour use the same link system, X-band and Ka-band (when available) uplink and downlink. An additional observable is provided by the possibility of locking to the $\mathrm{X}$-band uplink an additional Ka-band carrier downlink as shown in the Riley report (Riley et al., 1990). This will allow essentially complete calibration of the plasma contribution to the noise, which is the crucial factor in two way experiments, especially during conjunction experiments, when the signal propagates through the heavily ionized and turbulent medium of the solar corona. This is a necessary condition for the coherent reconstruction of the signal over the long duration of the experiment.

\subsection{SpaCECRAfT ElEMENTS}

On the Cassini Orbiter, the radio science instrument is encompassed in the radio science subsystem (RSS). RSS is really a virtual subsystem in that it is composed of elements from three spacecraft subsystems, two of which have other functions to perform as well. The subsystems that participate in RSS are the RFIS, the RFS, and the Antenna Subsystem. Figure 11 illustrates the elements of the subsystems that make up RSS, and the RF paths through the instrument that are involved in 

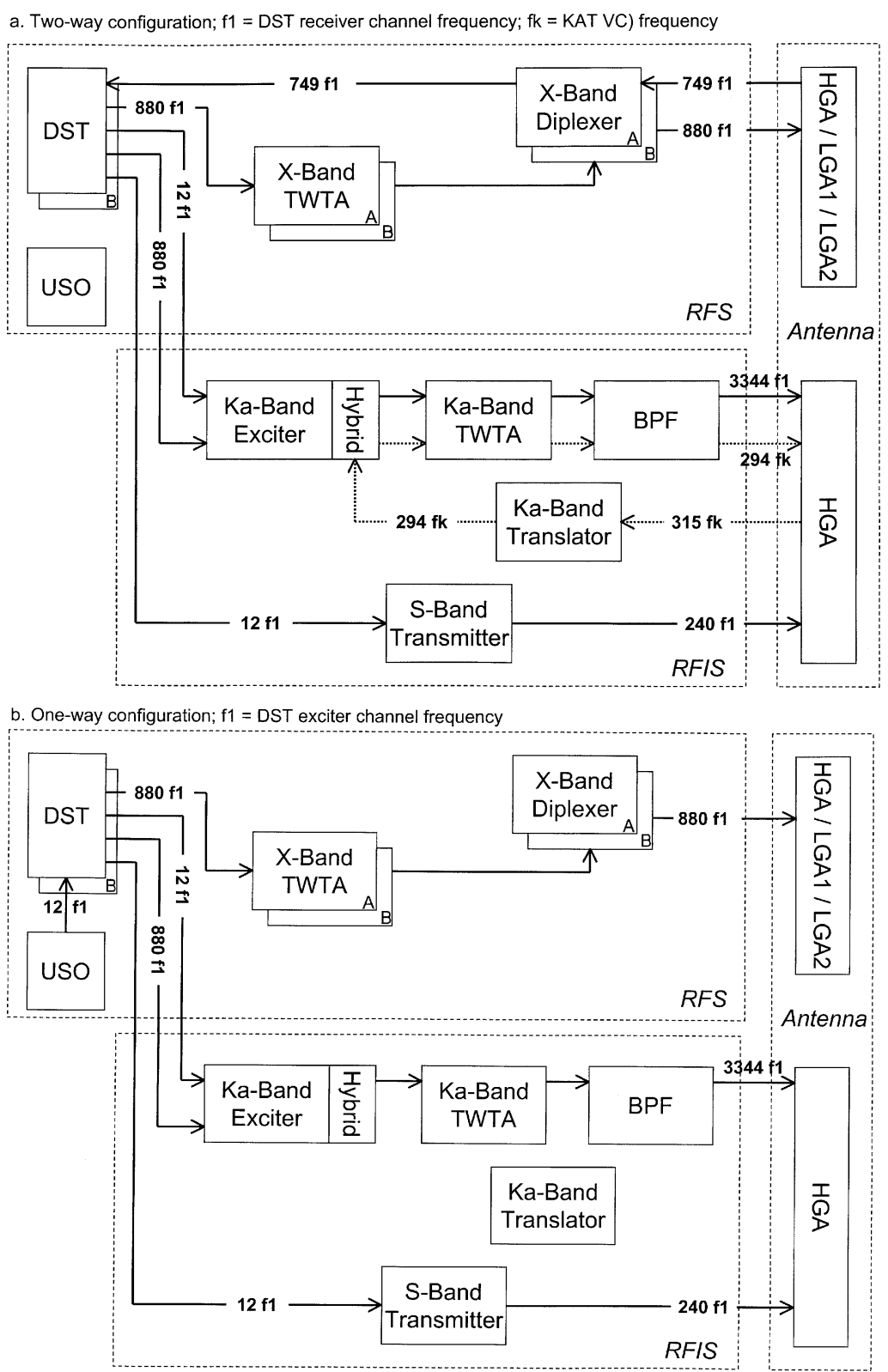

Figure 11. Radio frequency paths through the Cassini Orbiter radio science subsystem for two-way configuration (a) and one-way configuration (b).

its various operational modes. Figure 11a shows the signal paths available when the instrument is in two-way configuration, while Figure 11b shows those for the one-way configuration. In each case the active signal paths and subassemblies are depicted in bold. 


\subsubsection{Radio Frequency Subsystem}

The primary function of the RFS, a redundant critical engineering subsystem, is to support spacecraft telecommunications. RFS receives commands and transmits telemetry at X-band; its carrier is also the source of the X-band downlink signals used by radio science. For radio science purposes, the key components are the USO, the deep space transponders (DSTs) and the X-band traveling wave tube amplifiers (X-TWTAs).

The RFS has three modes of operation:

- two-way coherent mode, where the receiver in the DST is locked to the uplink and the downlink is referenced to, or coherent with, that uplink;

- two-way noncoherent mode, where the receiver is locked to the uplink and the exciter in the DST is referenced to either the USO or the transponder's auxiliary oscillator; and

- one-way mode, where there is no uplink and the exciter is referenced to either the USO or the transponder's auxiliary oscillator.

When RSS is operating in two-way mode, RFS is in its two-way coherent mode. The active DST receives a $7.2 \mathrm{GHz}$ uplink signal from the HGA or one of the two low gain antennas (LGAs), amplifies it, and translates it by the ratio 880/749 into an $8.4 \mathrm{GHz}$ downlink. The precise frequency of the uplink of course depends on the Doppler shift experienced in its transit from the ground. The DSTs are capable of locking onto a signal as low as $-155.8 \mathrm{dBm}$. The DST also generates input signals, coherent with the uplink, for the S-band transmitter (SBT) and the Ka-band exciter (KEX) in the RFIS. For the SBT, a single input at $115 \mathrm{MHz}$ is supplied. The KEX receives input at $8.4 \mathrm{GHz}$ and a reference signal at 115 MHz.

For one-way radio science experiments, RFS will be in either one-way or twoway noncoherent mode. The USO (the DST's auxiliary oscillator is not stable enough to use for radio science) provides a $115 \mathrm{MHz}$ input to the DST exciter, which multiplies it to $8.4 \mathrm{GHz}$ for the X-band downlink and for the $8.4 \mathrm{GHz}$ input to the KEX. The SBT input and the $115 \mathrm{MHz}$ reference signal for the KEX are sent directly from the USO.

Cassini's USO is a crystal oscillator based on a carefully selected $4.8 \mathrm{MHz}$ quartz resonator, which is housed along with its oscillator circuit and oven control circuit in a titanium Dewar whose internal temperature is maintained constant to within $0.001^{\circ} \mathrm{C}$ by a proportionally controlled oven. The USO's $114.9 \mathrm{MHz}$ output provides a reference signal for the DST, SBT, and KEX that has exceptional shortterm phase and frequency stability.

The X-band downlink from the DST is amplified by the X-TWTA to $15.8 \mathrm{~W}$, and radiated to earth through the HGA or whichever of the two LGAs is active. $\mathrm{X}$-band is the only frequency available to radio science when the spacecraft is not using the HGA. 


\subsubsection{Radio Frequency Instrument Subsystem}

The elements in the RFIS are devoted exclusively to radio science. They include the SBT, the KEX, the Ka-band Translator (KAT) and the Ka-band TWTA (K-TWTA). Primary application of the SBT and the KEX is in one-way experiments, though the KEX will also be used in two-way (X-up, Ka-down) mode. The SBT transmits a $13.5 \mathrm{~W}, 2.3 \mathrm{GHz}$ carrier derived from the $115 \mathrm{MHz}$ reference provided by the DST, through a diplexer contained in the probe receiver front end (RFE), to the HGA. The KEX multiplies its $115 \mathrm{MHz}$ reference by $11 / 3$, mixes the result with its 8.4 $\mathrm{GHz}$ input, and multiplies by 4 to produce $32 \mathrm{GHz}$. That output is routed through a hybrid coupler contained in the KEX subassembly to the K-TWTA.

Used only in two-way mode, the KAT turns a $34 \mathrm{GHz}$ uplink signal from the HGA into a coherent $32-\mathrm{GHz}$ downlink using a $14 / 15$ translation ratio. The translator is capable of generating a downlink with 1000-second Allan deviation of $3 \times 10^{-15}$ from input signals as low as $-132 \mathrm{dBm}$. KAT output goes through the hybrid coupler in the KEX subassembly and then to the K-TWTA . Amplification of Ka-band output from both the KEX and the KAT, singly or simultaneously, is accomplished in the K-TWTA. The amplifier produces a total output power of $7.2 \mathrm{~W}$ when operating with one carrier and $5.7 \mathrm{~W}$ in dual-carrier mode. Like the SBT, it feeds the HGA only.

\subsubsection{High Gain Antenna}

While all the Cassini Orbiter antennas are usable at X-band, only the HGA transmits all the radio science frequencies. In addition to supporting telecommunications and radio science, the HGA also serves as the transmit and receive antenna for the Cassini Radar and for the probe relay link. It is the most complex antenna ever flown on a planetary spacecraft, functioning at S-band, X-band, Ka-band, and Kuband. Its functions are illustrated in Figure 12. The portions used for radio science experiments or in-flight tests are highlighted.

Carrier signals transmitted between the spacecraft and the ground are all circularly polarized. X-band signals are received and transmitted with right-hand circular polarization through the RFS A-string diplexer and with left-hand circular polarization through the B-string diplexer. The Ka-band uplink is left-hand circularly polarized, the downlink right-hand circularly polarized. The S-band downlink is right-hand circularly polarized. The S-band transmit signal path is shared between the probe relay link and radio science.

\subsection{GROUND ELEMENTS}

\subsubsection{Deep Space Network}

The DSN comprises three complexes around the globe, called the deep space communication complexes (DSCC), located in the desert of Southern California, near Madrid, Spain, and near Canberra, Australia. Each complex is equipped with several tracking stations of different aperture size and different capabilities. Each 
ANTENNA SUBSYSTEM FUNCTIONAL BLOCK DIAGRAM

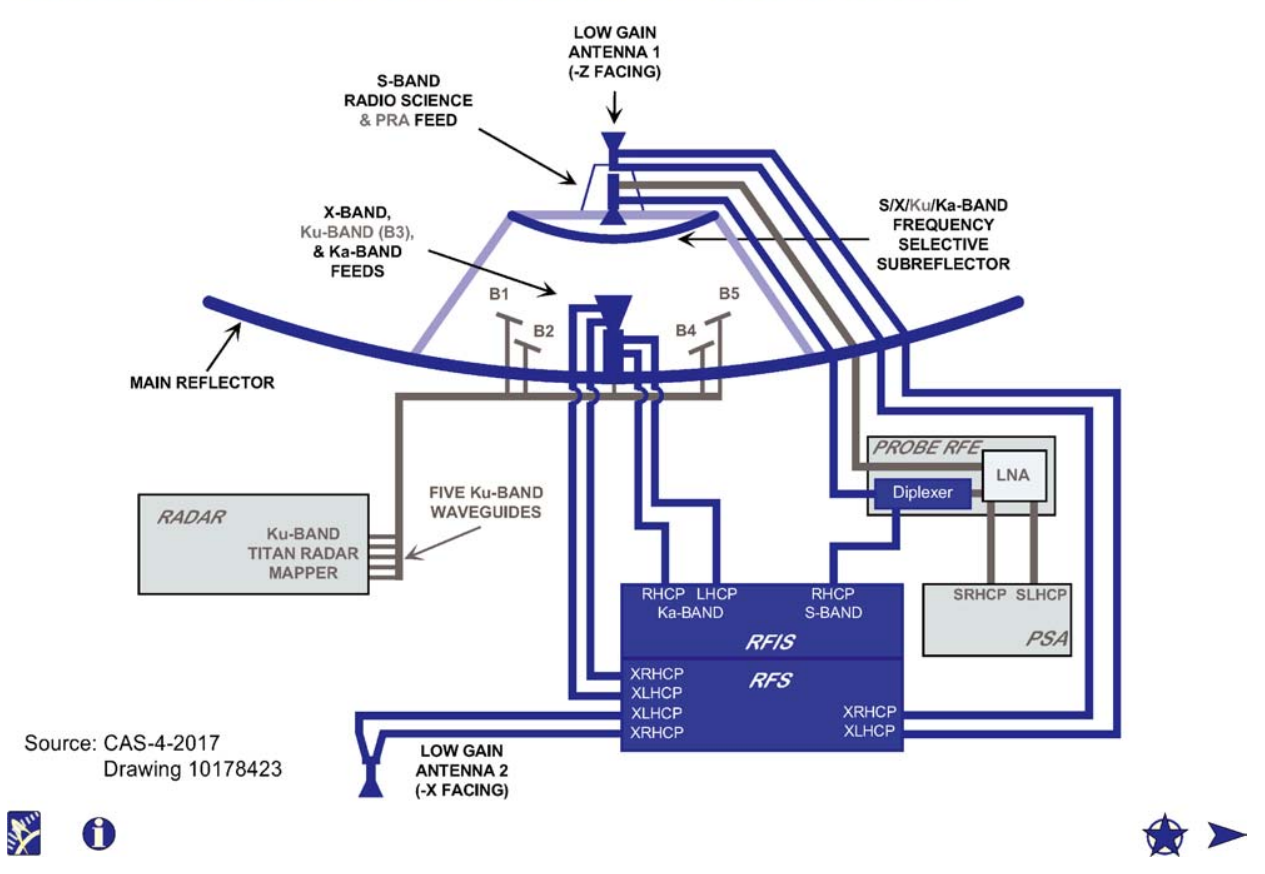

Figure 12. The Cassini antenna subsystem, with radio science functions emphasized.

complex has one 70-m diameter station, one 34-m high-efficiency (HEF) station, and at least one 34-m beam-wave-guide (BWG) station. Though their primary functions are to send commands to and to receive telemetry from space probes, these complexes have been designed to be an integral part of the radio science instrument (Asmar and Renzetti, 1993). As such, their performance and proper calibration directly determine the accuracy of Radio science experiments. The following subsystems of the DSN stations are relevant to acquisition of radio science data (see Figure 13): the monitor and control subsystem, the antenna mechanical subsystem, microwave subsystem, receiver-exciter subsystem, transmitter subsystem, tracking subsystem, spectrum processing subsystem, and frequency and timing subsystem.

The monitor and control subsystem receives and archives information sent to the complex from the control center at JPL. It also handles and displays responses to directives for configuration or information. Operator control consoles at the complex central processing center allow centralized control of all station subsystems within that complex. The center receives and distributes schedules of activities, sequence of events, prediction files for pointing the antennas and tuning the receivers, and other information.

The antenna surface performs two functions. As part of the receiving function, it acts as a large aperture to collect incoming energy transmitted from a spacecraft and 


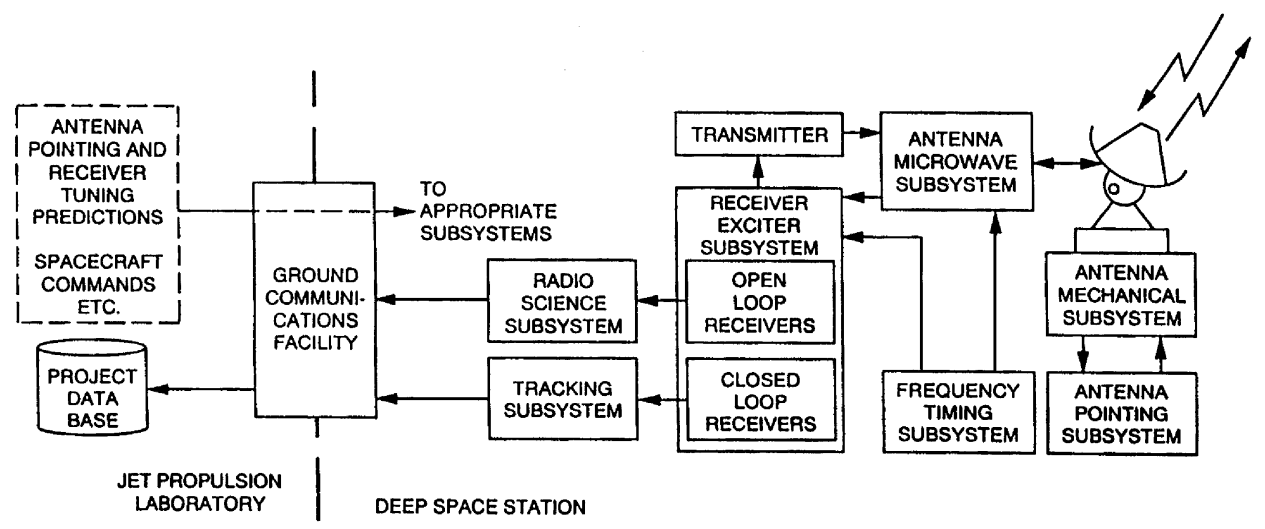

Figure 13. DSN subsystems relevant to the acquisition of radio science data.

focus it onto the feed horns. The subreflector is adjustable in the axial and angular positions in order to correct for gravitational deformation due to the motion of the antenna between zenith and the horizon. The primary surface is a paraboloid, modified for optimized illumination and signal stability. As part of the transmitting function, the antenna surface is used to form a narrow microwave beam that is directed to the spacecraft.

The electronics handles the received signal in two main steps. First the microwave subsystem accepts the S-, X-, and/or Ka-bands (depending on the station) and directs them via polarizer plates and microwave mirrors to low-noise amplifiers. Then the amplified signals are downconverted by local oscillators and routed to the receivers. These two processes set the electronics contribution to the overall amplitude sensitivity (usually measured as system noise temperature) and frequency/phase stability (generally quantified in terms of Allan deviation and phase noise respectively).

Two types of receivers (part of the receiver-exciter system) can be used: closedloop and open-loop. (The distinction is that the closed-loop receiver estimates parameters such as phase and amplitude in real time with standardized values of receiver bandwidths and time constants; the open-loop receivers record the predetection electric field for subsequent non-real time processing.) The closed-loop receiver (Block V Receiver) is the primary DSN receiver for telemetry and tracking data. It phase-locks to the signal carrier and demodulates science data, engineering data, and ranging signals transmitted by the spacecraft. The tracking subsystem measures Doppler shifts and ranging information based on the closed-loop receiver output.

The open-loop receiver (called the radio science receiver) downconverts and digitizes a selected bandwidth of the spectrum centered around the carrier signal. It utilizes a fixed first local oscillator and a tunable second local oscillator that is driven by a tuning "predict set" (predicted downlink frequency versus time) that 
takes into account the relative motion between the spacecraft and ground station. The predict set can also take into account the atmospheric effects of the planet under study. With no real-time signal detection requirements, the open-loop system provides flexibility in post-processing of the data. It is also designed with stringent requirements on amplitude, frequency and phase noise stability. Additional advantages of the open-loop reception include the simultaneous handling of signals in two polarization states as well as capturing multiple signals resulting from multipath propagation between the spacecraft transmission and the earth reception.

The transmitter subsystem utilizes a frequency reference to synthesize the uplink frequency channel assigned to a specific spacecraft. It also has the capability to tune the uplink to account for the Doppler shift on the uplink. The DSN can transmit at S-, $\mathrm{X}$ - or Ka-bands depending on the transmitting station and the receiving spacecraft. A record of the transmitted frequencies is saved for post-processing of the two-way Doppler observable, as necessary.

The frequency and timing subsystem provides a reference that drives the local oscillator devices throughout the complex. Global positioning system (GPS) satellites are utilized for inter-complex timing calibration. Currently centered on hydrogen masers, the frequency and timing system will be upgraded to include additional devices to meet the Cassini requirements including a linear trapped ions standard with a cryogenic local oscillator and a feedback-stabilized frequency distribution. Performance of the frequency and timing subsystem to fractional frequency stabilities of order $10^{-15}$ for integration times of $1000 \mathrm{~s}$, as well as phase noise lower than $-75 \mathrm{dBc}$ at $100 \mathrm{~Hz}$ from a Ka-band carrier, is fundamental to many radio science observations.

\subsubsection{0-m Stations}

The 70-m diameter stations of the DSN (DSS-14, DSS- 63, and DSS-43) are currently equipped for transmission at the S-band frequencies and reception at $\mathrm{S}$ - and $\mathrm{X}$-band frequencies. Future upgrades to transmit at X-band are possible. The microwave subsystem allows reception at both polarizations such that the right circular polarization (RCP) and left circular polarization (LCP) components of each band can be received via the radio science System. Accurate pointing of the 70-m stations is achieved by either active conical scanning or via blind pointing, driven by predicts of the spacecraft's position on the sky. It is anticipated that the 70-m stations will be used for occultation and other experiments requiring S-band reception from the Cassini spacecraft. The new BWG stations in Spain and Australia also may be used for occultation measurements.

\subsubsection{Beam Wave-Guide Stations}

The 34-m diameter BWG stations have been recently added to the DSN. Several BWG stations will exist at the California DSCC and one station each will be at the Spanish and Australian complexes. It is anticipated that only one BWG station will be instrumented for transmission and reception at Ka-band frequencies. This 
station in California (DSS-25) was developed to meet stringent Radio Science requirements on all of its components, including special structural and pointing requirements unique to a high-precision two-way Ka-band capability. In time for Saturn's tour, it is expected that Ka-band downlink will be available at 34-m BWG stations DSS-54 and DSS-34 in Spain and Australia, respectively.

\subsubsection{High-Efficiency Stations}

The 34-meter diameter high-efficiency DSN stations (DSS-15, DSS-65, and DSS45) are equipped for transmission at X-band and are optimized for reception at $\mathrm{X}$-band. The microwave and receiver subsystems allow for the reception of two channels simultaneously. The station pointing capabilities and strategies are similar to those of the $70-\mathrm{m}$ stations.

\subsubsection{Media Calibration System}

The earth's atmosphere contributes phase and amplitude noise to a spacecraft radio signal received at a ground station. A system to calibrate the effects of the atmosphere on the phase of the microwave signal is under development especially for Cassini radio science experiments utilizing $\mathrm{Ka}$-band, where excellent end-to-end frequency and phase stability is required. The purpose of this media calibration system is to provide a line-of-sight calibration of the water vapor delay (responsible for most of the atmosphere induced phase fluctuations at microwave frequencies) during DSS-25 radio science experiment passes. It will also provide estimates of the total zenith delay and delay fluctuation. This will be accomplished via several components of the system. An advanced water vapor radiometer will sense the number of water vapor molecules along the line-of-sight, a microwave temperature profiler will sense the vertical temperature distribution, a surface meteorology package will measure the temperature, pressure, and humidity, and a GPS receiver will provide the total zenith delay estimates.

\subsubsection{Other Stations}

The utility of stations other than those of NASA's DSN has been proven for several radio science experiments on past missions. For example, arraying of different antennas has improved the signal strength coming from an extremely distant spacecraft (as during the Voyager Neptune encounter, where Australian and Japanese stations also recorded the received signals in collaboration with the ight project and science team.)

More recently, the contribution of an Italian station and a Japanese station has proven valuable to the radio science experiments on the Ulysses mission. These stations are normally equipped with a dual S- and X-bands receiver and a hydrogen maser frequency standard in order to perform very long baseline interferometry studies. Adding a dedicated instrument to these radio telescopes made it possible to measure the phase and amplitude of the S- and X-band carrier signals transmitted from Ulysses. This instrument, called a digital tone extractor, has been used at 
both the Medicina 32-m parabola in Italy and at the Kashima observatory in Japan. Medicina successfully participated in the three measuring campaigns of the 1991, 1992 and 1993 opposition and conjunction experiments made with Ulysses. The operation started with the transfer of trajectory information from JPL to the station where pointing coordinates and the downlink Doppler shifts were computed. The acquired data were comparable in quality to the DSN data for those experiments.

The information collected at these two stations enabled the sampling of a propagation path across a substantially different region of the troposphere and ionosphere than those of the DSN stations. Furthermore, the data were useful in understanding the DSN-acquired data at times of unusual behavior of the signal or equipment.

The availability of non-DSN stations, capable of operation up to Ka-band and available for limited times during specific mission events, has become crucial since existing plans allow for only one DSN station (DSS-25, discussed earlier) expressly dedicated to the Cassini radio science experiments at Ka-band. The possible future availability of an Italian station, located more than $120^{\circ}$ away in longitude from Goldstone, would almost double the tracking time at Ka-band for Cassini. This could significantly improve the integration time for gravitational wave experiments, as well as benefit other radio science research. Availability of such non-DSN stations might open new opportunities during the cruise and tour phase of the Cassini mission, in addition to reducing the tracking load on the DSN under critical conditions.

\section{Conclusion}

At the time this paper is being written, a partial check-out of the radio science instrument indicates very good performance, and scientific data were obtained during the flybys of Venus and Earth. The investigators are working at planning future observations and looking forward to successful cruise and tour experiments.

\section{Acknowledgments}

The Radio Science instrumentation on the Cassini Orbiter represents a truly global effort, with contributions from organizations all over the world. The DST was developed for JPL by Motorola. Both X-band and Ka band TWTAs were provided by Hughes Electronics; the Ka band TWTA utilized a design developed from research done at NASA Lewis Research Center. The USO was designed and built by the Johns Hopkins University Applied Physics Laboratory. The KEX was designed and built at JPL. The SBT and KAT were provided by the Italian firm Alenia Spazio for our partner, the Italian Space Agency (ASI), and Alenia Spazio integrated and tested the RFIS. The Radio-Science Team wishes to particularly thank B. Bertotti, now retired from the Radio Science Team, for his important efforts leading to Cassini's superb radio system, his work on the cruise experiments, and his work in preparation for the Tour. 
We also thank the Cassini engineers in JPL's Radio Science Systems Group: Aseel Anabtawi, Elias Barbinis, Don Fleischman, Gene Goltz, Randy Herrera, Trina Ray, and the personnel of the DSN for their efforts and support of the experiments described in this paper. The Radio Science Team thanks G. Comoretto, F. B. Estabrook, J. Lunine, M. Tinto, and R. Woo for discussions and collaborations.

\section{References}

Achterberg, R. K. and Flasar, F. M.: 1996, Icarus 119, 359-369.

Allison, M. D.: 1990, Icarus 83, 282-307.

Anderson, J. D., Armstrong, J. W., and Lau, E. L.: 1993, ApJ 408, 287-292.

Anderson, J. D., Sjogren, W. L., and Schubert, G.: 1996a, Science 272, 709-712.

Anderson, J. D., Lau, E. L., Sjogren, W. L., Schubert, G., and Moore, W. B.: 1996b, Nature 384, 541-543.

Anderson, J. D., Lau, E. L., Sjogren, W. L., Schubert, G., and Moore, W. B.: 1997a, Nature 387, 264-266.

Anderson, J. D., Lau, E. L., Sjogren, W. L., Schubert, G., and Moore, W. B.: 1997b, Science 276, 1236-1239.

Armstrong, J. W.: 1989, Schutz, B. (ed.), Gravitational Wave Data Analysis, Kluwer, Dordrecht, $153-172$

Armstrong, J. W.: 1998, Radio Science 33, 1727-1738.

Armstrong, J. W., Iess, L., Tortora, P., and Bertotti, B.: 2003, Astrophysics J. 599, 806-813.

Asmar, S. W., and Renzetti, N. A.: 1993, JPL Publication 80-93 Rev. 1, Jet Propulsion Laboratory, Pasadena, California.

Aswar, S. W., Armstrong, J. W., Iess, L., and Tortora, P.: 2004, Radio Science (submitted).

Augstein, E.: 1978, Shaw, D. B. (ed.) Meteorology Over the Tropical Oceans, Royal Meteorological Society, pp. 73-103.

Bender, P., Brillet, A., Ciufoloni, I., Cruise, A. M., et al.: 1998, Pre-Phase A Report (Second Edition), Max-Planck-Institut fur Quantenoptik, Garching, MPQ 233.

Bertotti B., and Giampieri, G.: 1992, Class. Quantum Grav. 9, 777-793.

Bertotti B., Comoretto, G., and Iess, L.: 1993, Astron. Astrophys. 269, 608-616.

Bertotti, B., Ambrosini, R., Armstrong, J. W., Asmar, S., Comoretto, G., Giampieri, G. et al.: 1995, Astron. Astrophys. 296, 13- 25.

Bertotti, B.: 1997, Francaviglia, M., et al. (eds.), Proceedings of 14th International Conference on General Relativity and Gravitation, World Scientific, Singapore, New Jersey, London, Hong Kong, pp. 79-101.

Bertotti, B., Iess, L., and Tortora, P.: 2003, A test of general Relativity using Radio Links with the Cassini spacecraft, Nature, 425, 374-376.

Bertotti B., and Giampieri, G.: 1998, Solar Physics 178, 85-107.

Bertotti B., Vecchio, A., and Iess, L.: 1999, Phys. Rev. D 5908(8), 2001.

Bird, M. K., Dutta-Roy, R., Asmar, S. W., and Rebold, T. A.: 1997, Icarus 130, 426-436.

Borderies, N., Balmino, G., Castel, L., and Moynot, B.: 1980, Moon Planet. 22, 191-200.

Borderies, N., Goldreich, P., and Tremaine, S.: 1982, Science 299, 209-211.

Borderies, N., Goldreich, P., and Tremaine, S.: 1983a, Astron. J. 88, 226-228.

Borderies, N., Goldreich, P., and Tremaine, S.: 1983b, Astron. J. 88, 1560-1568.

Borderies, N., Goldreich, P., and Tremaine, S.: 1983c, Icarus 53, 84-89.

Borderies, N., Goldreich, P., and Tremaine, S.: 1983d, Icarus 55, 124-132.

Borderies, N., Goldreich, P., and Tremaine, S.: 1984a, Astrophys. J. 284, 429-434. 
Borderies, N., Goldreich, P., and Tremaine, S.: 1984b, Greenberg, R. and Brahic, A., (eds.)., Planetary Rings, University of Arizona Press, Tucson, pp. 713-734.

Borderies, N., Goldreich, P., and Tremaine, S.: 1985, Icarus 63, 406-420.

Borderies, N., Goldreich, P., and Tremaine, S.: 1986, Icarus 68, 522-533.

Borderies, N., and Longaretti, P.-Y.: 1987, Icarus 72, 593-603.

Borderies, N., Goldreich, P., and Tremaine, S.: 1988, Icarus 80, 344-360.

Borderies, N.: 1992, Ferraz-Mello, S., (eds.), Proceedings of IAU Symposium No. 152, 'Chaos, Resonance and Collective Dynamical Phenomena in the Solar System' (Angra Dos Reis, Brazil, July 15-19, 1991), Kluwer Academic Publishers: Dordrecht, pp. 53-64

Borderies-Rappaport, N., and Longaretti, P.-Y.: 1994, Icarus 107, 129-141.

Bosh, A. S. and Olkin, C. B.: 1996, Bull. Am. Astron. Soc. 28, 1124-1124.

Broadfoot, A. L., et al.: 1981, Science 212, 206-211.

Brouwer, D. and Clemence, G. M.: 1961, Methods of Celestial Mechanics, Academic Press, New York.

Buratti, B. J.: 1988, Icarus 75, 113-126.

Caldwell, J.: 1977, Burns, J., (ed.), Planetary Satellites, University of Arizona Press, Tucson, pp. 438-450.

Campbell, J. K., and Anderson, J. D.: 1989, Astron. J. 97, 1485-1495.

Capone, L. A., Whitten, R. C., Dubach, J., Prasad, S. S., and Huntress, W. T.: 1976, Icarus 28, 367.

Cassen, P. M., Peale, S. J., and Reynolds, R. T.: 1982, Morrison, D., (ed.), Satellites of Jupiter, University of Arizona Press, Tucson, pp. 193-128.

Cohen, E. R., and Taylor, B. N.: 1987, Rev. Mod. Phys. 59, 1121-1148.

Conrath, B. J., Gautier, D., Hanel, R. A., and Hornstein, J. S.: 1984, Astrophys. J. 282, 807-815.

Conrath, B. J., and Gautier, D.: 2000, Icarus 144, 124-134.

Conrath, B. J., and Pirraglia, J. A.: 1983, Icarus 53, 286-292.

Courtin, R., Gautier, D., and McKay, C. P.: 1995, Icarus 114, 114-162.

Coustenis, A., Lellouch, E., Maillard, J.-P., and McKay, C. P.: 1994, Bull. Am. Astron. Soc. 26, 1181.

Coustenis, A., and B. Bézard: 1995, Icarus 115, 126-140.

Cravens, T. E.: 1987, J. Geophys. Res. 92, 11083.

Cuzzi, J. N., Lissauer, J. J., and Shu, F. H.: 1981, Nature 292, 703-707.

Cuzzi, J. N., Lissauer, J. J., Esposito, L. W., Holberg, J. B., Marouf, E. A., Tyler, G. L. et al.: 1984, Greenberg, R. and Brahic, A., (eds.), Planetary Rings, University of Arizona Press, Tucson, pp. 73-199.

Cynn, H. C., Boone, S., Koumvakalis, A., Nicol, M., and Stevenson, D. J.: 1989, Proceedings of the 19th Lunar and Planetary Science Conference, pp. 443-441.

Damour T. and Nordtvedt, K.: 1993, Phys. Rev. Lett. 70, 2217-2219.

Danielson, R. E., Caldwell, J., and Larach, D. R.: 1973, Icarus 20, 437-443.

Davies, M. E., Abalakin, V. K., Lieske, J. H., Seidelmann, P. K., Sinclair, A. T., Sinzi, A. M. et al.: 1983, Cel. Mech. 29, 309-321.

Davies, M. E., Abalakin, V. K., Bursa, M., Lieske, J. H., Morando, B., Morrison, D. et al.: 1996, Cel. Mech. 63, 127-148.

Dermott, S. F., and Thomas, P. C.: 1994, Icarus 109, 241-257.

Elliot, J. L., Frogel, J., Elias, J., Glass, I., French, R. G., Mink, D. J. et al.: 1981, Astron. J. 86, $127-134$.

Elliot, J. L., French, R. G., Frogel, J., Elias, J., Mink, D. J., and Liller, W.: 1981, Astron. J. 86, 444455.

Elliot, J. L., Elias, J. H., French, R. G., Frogel, J., Liller, W., Matthews, K. et al.: 1983, Icarus 56, 202-208.

Elliot, J. L., French, R. G., Meech, K. J., and Elias, J. H.: 1984, Astron. J. 89, 1587-1603.

Elliot, J. L., Glass, I. S., French, R. G., and Kangas, J. A.: 1987, Icarus 71, 91-102. 
Elliot, J. L., Bosh, A. S., Cooke, M. L., Bless, R. C., Nelson, M., Percival, J. W. et al.: 1992, EOS Trans. Am. Geophys. U. 73(14), 176.

Eshleman, V. R., Tyler, G. L., Wood, G. E., Lindal, G. F., Anderson, J. D., Levy, G. S., et al.: 1979a, Science 204, 976-978.

Eshleman, V. R., Tyler, G. L., Wood, G. E., Lindal, G. F., Anderson, J. D., Levy, G. S., et al.: 1979b, Science 206, 959-962.

Eshleman, V. R.: 1983, Science 221, 361-364.

Eshleman, V. R., Breakwell, J. V., Tyler, G. L, and Marouf, E. A: 1983, Icarus 54, 212-226.

Esposito, L. W., Borderies, N., Goldreich, P., Cuzzi, J. N., Holberg, J. B., Lane, A. L., Pomphrey, R. B., Terrile, R. J., Lissauer, J. J., Marouf, E. A., and Tyler, G. L.: 1983, Science 222, 57-60.

Esposito, L. W., Cuzzi, J. N., Holberg, J. B., Marouf, E. A., Tyler, G. L., and Porco, C. C.: 1984, Gehrels, T. and Matthews, M. S., (eds.)., Saturn, University of Arizona Press, Tucson, pp. 463-545.

Esposito, L. W., Brahic, A., Burns, J. A., and Marouf, E. A.: 1991, Uranus, pp. 439-447.

Estabrook, F. B., and Wahlquist, H. D.: 1975, Gen. Rel. Grav. 6, 439-447.

Estabrook, F. B.: 1978, Neugebauer, M. and Davies, R. W, (eds.), A Close-Up of the Sun, JPL Publication 78-70, Pasadena, pp. 441-449.

Eubanks, T. M., Matsakis, D. N., Martin, J. O., Archinal, B. A., Fey, A. L., Josties, F. J., et al.: 1997, Joint APS/AAPT Meeting, Washington April, D.C. 18-21, Paper K11.05.

Fjeldbo, G.: 1964, Report No. SU-64-025, Stanford Electronics Laboratories, Stanford, CA.

Fjeldbo, G., Eshleman, V. R., Garriott, O. K., and Smith, F. L, III: 1965, J. Geophys. Res. 70, 37013710.

Fjeldbo, G., and Eshleman, V. R.: 1968, Planet. Space Sci. 16, 1035-1059.

Fjeldbo, G., Kliore, A. J., and Eshleman, V. R.: 1971, Astron. J. 76, 123.

Fjeldbo, G., Kliore, A. J., Seidel, B. L., Sweetnam, D. N., and Cain, D. L.: 1975, Astron. and Astrophys. 39, 91-96.

Fjeldbo, G., Kliore, A. J., Sweetnam, D. N., Esposito, P. B., Seidel, B. L., and Howard, H. T.: 1976a, Icarus 29, 439-444.

Fjeldbo, G., Kliore, A. J., Seidel, B. L., Sweetnam, D. N., and Woiceshyn, P. M.: 1976b, Gehrels, T. (ed.), Jupiter, The University of Arizona Press, Tucson, Arizona, pp. 238-246.

Flasar, F. M., Samuelson, R. E., and Conrath, B. J.: 1981, Nature 292, 693-698.

Flasar, F. M.: 1983, Science 221, 55-57.

Flasar, F. M.: 1998a, Planet Space Sci. 46, 1109-1124.

Flasar, F. M.: 1998b, Planet Space Sci. 46, 1125-1147.

Flasar, F. M., Hinson, D. P., Kliore, A. J., Schinder, P. J., Twicken, J. D., Herrera, R. G., et al.: 1998, 'Galileo radio occultation measurements of Jupiter's highly variable Ionosphere', in International Symposium The Jovian System after Galileo - The Saturnian System before Cassini-Huygens, Nantes, France.

Flasar, F. M. et al.: 2004, Exploring the Saturn system in the thermal infrared: The composite infrared spectrometer. Space Sci. Rev. 115, 169-297.

Fox, J. L., and Yelle, R. V.: 1997, Geophys. Res. Lett. 24, 2179.

French, R. G., Elliot, J. L., and Allen, D. A.: 1982, Nature 298, 827-828.

French, R. G., Kangas, J. A., and Elliot, J. L.: 1986a, Science 231, 480-483.

French, R. G., Elliot, J. L., and Levine, S. E.: 1986b, Icarus 67, 134-163.

French, R. G., Jones, T. J., and Hyland, A. R.: 1987, Icarus 69, 499-505.

French, R. G., Elliot, J. L., French, L. M., Kangas, J. A., Meech, K. J., Ressler, M. E., et al.: 1988, Icarus 73, 349-378.

French, R. G., Clark, M. A., Tollestrup, E., Robinson, E., Harvey, P., Heilman, L., et al.: 1989, Bull. Am. Astron. Soc. 21, 928.

French, R. G., Chanover, N. J., Clark, M. A., Tollestrup, E., and Baron, R. L.: 1990, Bull. Am. Astron. Soc. 22, 1069-1070. 
French, R. G., Nicholson, P. D., Porco, C. C., and Marouf, E. A.: 1991, Uranus, 327-409.

French, R. G., Nicholson, P. D., Cooke, M. L., Elliot, J. L., Matthews, K., Perković, O., et al.: 1993, Icarus 103, 163-214.

Friedson, A. J.:1994, Icarus 109, 40-57.

Gan, L., Keller, C. N., and Cravens, T. E.: 1992, J. Geophys. Res. 97, 12.

Gautier, D., et al. 1981.

Goldreich, P., and Tremaine, S.: 1978a, Icarus 34, 240-253.

Goldreich, P., and Tremaine, S.: 1978b, Astrophys. J. 222, 850-858.

Goldreich, P., and Tremaine, S.: 1979a, Astrophys. J. 233, 857-871.

Goldreich, P., and Tremaine, S.: 1979b, Astron. J. 84, 1638-1641.

Goldreich, P., and Tremaine, S.: 1979c, Nature 277, 97-99.

Goldreich, P., and Tremaine, S.: 1980, Astrophys. J. 241, 425-441.

Goldreich, P., and Tremaine, S.: 1981, Astrophys. J. 243, 1062-1075.

Goldreich, P., Tremaine, S., and Borderies, N.: 1986, Astron. J. 92, 490-494.

Goldreich, P., Rappaport, N., and Sicardy, B.: 1995, Icarus 118, 414-417.

Grasset, O., and Sotin, C.: 1996, Icarus 123, 101-112.

Gresh, D.L., Rosen, P. A., Tyler, G. L., and Lissauer, J. J.: 1986, Icarus 68, 481-502.

Gresh, D. L., Marouf, E. A., Tyler, G. L., Rosen, P. A., and Simpson, R. A.: 1989, Icarus 78, 131-168. Griffith, C. A.: 1993, Nature 364, 511-514.

Griffith, C. A., and Zahnle, K.: 1995, Geophys. J. Res. 100, 16, 907-16,922.

Grossman, A. W., Muhleman, D. O, and Berge, G. L.: 1990, Science 245, 1211-1215.

Grossman, A. W., and Muhleman, D. O.: 1992, Bull. Am. Astron. Soc. 24, 954.

Guillot, T., Chabrier, G., Morel, P., and Gautier, D.: 1994, Icarus 112, 354-367.

Habbal, S. R., Woo, R., Fineschi, S., O’Neal, R., Kohl, J., Noci, G., et al.: 1997, Astrophys. J. 489, L103-L106.

Han, B., and Owen, T.: 1994, Bull. Am. Astron. Soc. 26, 1181.

Hanel, R. A., Conrath, B., Flasar, F. M., Kunde, V., Maguire, W., Pearl, J., Pirraglia, J., Samuelson, R., Herath, L., Allison, M., Cruikshank, D., Gautier, D., Gierasch, P., Horn, L., Koppany, R., and Ponnamperuma, C.: 1981, Science 212, 192-200.

Hanel, R. A., Conrath, B. J., Kunde, V. G., Pearl, J. C., and Pirraglia, J. A: 1983, Icarus 53, 262-285.

Hanninen, J., and Salo, H.: 1994, Icarus 108, 325-346.

Hanninen, J., and Salo, H.: 1995, Icarus 117, 435-438.

Haugstad, B. S.: 1978, Radio Sci. 13, 435-440.

Hinson, D. P., Flasar, F. M., Kliore, A. J., Schinder, P. J., Twicken, J. D., and Herrera, R. G.: 1977, Geophys. Res. Lett. 24, 2107-2110.

Hinson, D. P., and Tyler, G. L., et al.: 1983, Icarus 54, 337-352.

Hinson, D. P., and Magalhães, J. A.: 1991, Icarus 94, 64-91.

Hinson, D. P., and Magalhães, J. A.: 1993, Icarus 105, 142-161.

Hinson, D. P., Flasar, F. M., Kliore, A. J., Schinder, P. J., Twicken, J. D., and Herrera, R. G.: 1997, Geophys. Res. Lett. 24, 2107-2110.

Hinson, D. P., Twicken, J. D., and Karayel, E. T.: 1998a, J. Geophys. Res. 103, 9505-9520.

Hinson, D. P., Kliore, A. J., Flasar, F. M., Twicken, J. D., Schinder, P. J., and Herrera, R. G, 1998, J. Geophys. Res. Space Phys. 103, 29343-29357.

Holberg, J. B., Nicholson, P. D., French, R. G., and Elliot, J. L.: 1987, Astron. J. 94, 178-188.

Howard, H. T., Tyler, G. L., Fjeldbo, G., Kliore, A. J., Levy, G. S., Brunn, D. L., et al.: 1974a, Science 183, 1297-1301.

Howard, H. T., Tyler, G. L., Fjeldbo, G., Kliore, A. J., Levy, G. S., Brunn, D. L., et al.: 1974b, Science 185, 179-180.

Hubbard, W. B., and Anderson, J. D.: 1978, Icarus 33, 336-341.

Hubbard, W. B.: 1984, Planetary Interiors, Van Nostrand Reinhold Company, New York 
Hubbard, W. B., Porco, C. C., Hunten, D. M., Rieke, G. H., Rieke, M. J., McCarthy, D. W., et al.: 1993, Icarus 103, 215-234.

Hubbard, W.B., Guillot, T., Marley, M. S., Burrows, A., Lunine, J. I., and Saumon, D. S: 1999, Plan. Space Sci., submitted.

Hunten, D. M.: 1978, Hunten, D. M and Morrison, D. (eds.), The Saturn System, NASA Conf. Publ. 2068, pp. 127-140.

Iess, L., and Armstrong, J. W.: 1997, Ciufolini, I. and Fidecaro, F. (eds.), Proceedings of the Conference on Gravitational Waves: Sources and Detectors, Cascina, Italy, 19-23 March 1996, World Scientific, 323-343

Iess, L., Giampieri, G., Anderson, J. D., and Bertotti, B.: 1999, Clas. Quantum Grav. 16, 14870-1502. Ip, W. H.: 1990, Astropys. J. 362, 354.

Jacobson, R. A.: 1996, Bull. Am. Astron. Soc. 28, 1185.

Jenkins, J. M., Steffes, P. G., Twicken, J. D., Hinson, D. P., and Tyler, G. L.: 1994, Icarus 110, 79-94.

Johnson, R. E., Killeen, R. M., Waite, J. H., Jr., and Lewis, W. S.: 1998, Geophys. Res. Lett. 25, $3,257-3,260$.

Jones, T. D., and Lewis, J. S.: 1987, Icarus 72, 381-393.

Karayel, E. T., and Hinson, D. P.: 1997, Radio Sci. 32, 411-423.

Kaula, W. M.: 1964, Rev. Geophys. 2, 661-685.

Keller, C. N., Cravens, T. E., and Gan, L.: 1992, J. Geophys. Res. 97, 12117-12135.

Keller, C. N., Cravens, T. E., and Gan, L.: 1994, J. Geophys. Res. 99, 6511-6525.

Keller, C. N., Anicich, V. G., and Cravens, T. E.: 1998, Planet. Space Sci. 46, 1157-1174.

Kliore, A. J., Cain, D. L., and Hamilton, T. W.: 1964, Determination of Some Physical Properties of the Atmosphere of Mars from Changes in the Doppler Signal of a Spacecraft on an EarthOccultation Trajectory, No, T. R.. 32-674, Jet Propulsion Laboratory, Pasadena, CA.

Kliore, A. J., Cain, D. L., Levy, G. S., Eshleman, V. R., Fjeldbo, G., and Drake, F. D.: 1965, Science 149, 1243-1248.

Kliore, A. J., Levy, G. S., Cain, D. L., Fjeldbo, G., and Rasool, S. I.: 1967, Science 158, 1683-1688.

Kliore, A. J., Fjeldbo, G., Seidel, B. L., and Rasool, S. I.: 1969, Science 166, 1393-1397.

Kliore, A. J., Cain, D. L., Fjeldbo, G., Seidel, B. L., and Rasool, S. I.: 1972a, Icarus 12, 484-516.

Kliore, A. J.: 1972b, Colin, L. (ed.), The Mathematics of Profile Inversion, NASA TM X-62,150, NASA Ames Research Center, Moffett Field, CA, pp. 3-2-3-17.

Kliore, A. J., Cain, D. L., Fjeldbo, G., Seidel, B. L., and Rasool, S. I.: 1974, Science 183, 323-324.

Kliore, A. J., Fjeldbo, G., Seidel, B. L., Sweetnam, D. N., Sesplaukis, T. T., Woiceshyn, P. M., et al.: 1975, Icarus 24, 407-410.

Kliore, A. J., Woiceshyn, P. M., and Hubbard, W. B.: 1976, Geophys. Res. Lett. 3, 113-116.

Kliore, A. J., Woiceshyn, P. M., and Hubbard, W. B.: 1977, Pergamon Press, Oxford and New York, pp. 703-710.

Kliore, A. J., Patel, I. R., Nagy, A. F., Cravens, T. E., and Gombosi, T. I.: 1979, Science 205, 99-102.

Kliore, A. J., Patel, I. R., Lindal, G. F., Sweetnam, D. N., Hotz, H. B., Waite, J. H., Jr., et al.: 1980a, J. Geophys. Res. 85, 5857-5870.

Kliore, A. J., and Patel, I. R.: 1980, J. Geophys. Res. 85, 7957-7962.

Kliore, A. J., Lindal, G. F., Sweetnam, D. N., Hotz, H. B., and McDonough, T. R.: 1980b, Science 207, 446-449.

Kliore, A. J., and Patel, I. R.: 1982, Icarus 52, 320-334.

Kliore, A. J., and Mullen, L. F.: 1989, J. Geophys. Res. 94, 13339-13351.

Kliore, A. J., and Luhmann, J. G : 1991, J. Geophys. Res. 96, 21281-21289.

Kliore, A. J., Hinson, D. P., Flasar, F. M., Nagy, A. F., and Cravens, T. E.: 1997, Science 277, 355.

Kliore, A. J.: 1998a, Andersen, J. (ed.), AndersenHighlights in Astronomy, International Astronomical Union, pp.1065-1069.

Kliore, A. J., Herrera, R. G., Hinson, D. P., Twicken, J. D., Flasar, F. M., and Schinder, P. J.: 1998b, 30th DPS Meeting, Madison, WI. 
Kolosov, M. A., Yakovlev, O. I., Yakovleva, G. D., Efimov, A. I., Trusov, B. P., Timofeyava, T. S., et al.: 1975, Cosmic Res. 13, 46.

Kolosov, M. A., Yakovlev, O. I., Matyugov, S. S., Timofeyava, T. S., Yakovleva, G. D., and Kalashnikov, I. E.: 1976, Cosmic Res. 16, 221.

Kozai, Y.: 1957, Ann. Tokyo Obs. Ser. 25, 73.

Lane, A. L., Hord, C. W., West, R. A., Esposito, L. W., Coffeen, D. L., Sato, M., Simmons, K. E., Pomphrey, R. B., and Morris, R. B.: 1982, Science 215, 537-543.

Lellouch, E., Coustenis, A., Gautier, D., Raulin, F., Dubouloz, N., and Frère, C.: 1989, Icarus 79, 328-349.

Lemmon, M. T., Karkoschka, E., and Tomasko, M.: 1993, Icarus 103, 329-332.

Lewis, J. S., and Prinn, R. G.: 1980, Astrophys. J. 238, 357-364.

Lin, D. N. C., and Bodenheimer, P.: 1981, Astrophys. J. Lett. 248, L83-L86.

Lindal, G. F., Hotz, H. B., Sweetnam, D. N., Shippony, Z., Brenkle, J. P., Hartsell, G. V., et al.: 1979, J. Geophys. Res. 84, 8443-8456.

Lindal, G. F., Wood, G. E., Levy, G. S., Anderson, J. D., Sweetnam, D. N., Hotz, H. B., et al.: 1981, J. Geophys. Res. 86, 8721-8727.

Lindal, G. F., Wood, G. E., Hotz, H. B., Sweetnam, D. N., Eshleman, V. R., and Tyler, G. L.: 1983, Icarus 53, 348-363.

Lindal, G. F., Sweetnam, D. N., and Eshleman, V. R.: 1985, Astron. J. 90, 1136-1146.

Lindal, G. F., Sweetnam, D. N., and Eshleman, V. R.: 1985a, J. Geophys. Res. 92, 14987-15001.

Lindal, G. F., Sweetnam, D. N., and Eshleman, V. R.: 1985b, Astron. J. 90, 1136-1146.

Lindal, G. F., Lyons, J. R., Sweetnam, D. N., Eshleman, V. R., D P. Hinson, and Tyler, G. L.: 1987, J. Geophys. Res. 92, 14987-15001.

Lindal, G. F.: 1992, Astron. J. 103, 967-982.

Lissauer, J. J., Shu, F. H., and Cuzzi, J. N.: 1982., Brahic, A., (ed.), Proceedings of IAU Colloquium 75 , 'Planetary Rings' (Toulouse, France, August 1992), Cepadues Editions, Toulouse, pp. 385-392.

Longaretti, P.-Y., and Borderies, N.: 1986, Icarus 67, 211-223.

Longaretti, P.-Y., and Borderies, N.: 1991, Icarus 94, 165-170.

Longaretti, P.-Y., and Rappaport, N.: 1995, Icarus 116, 376-396.

Lorenz, R. D., McKay, C. P., and Lunine, J. L.: 1997, Science 275, 642-644.

Love, A. E. H.: 1906, A Treatise on the Mathematical Theory of Elasticity, Cambridge University Press, Cambridge

Lukkari, J.: 1981, Nature 292, 433-435.

Lunine, J. I., and Stevenson, D. J.: 1987, Icarus 70, 61-77.

Lunine, J. I.: 1996, Ambrosini, R. (ed.)., Proceedings of The Cassini-Huygens Mission: The Exploration of the Saturn System,' (Bologna, Italy, November 19-22, 1996), Arti Grafiche S. Marcello srf: Roma, 14.

Majeed, T., and McConnell, J. C.: 1996, J. Geophys. Res. 101, 7589.

Marouf, E. A., Tyler, G. L. and Eshleman, V. R.: 1982, Icarus 49, 161-193.

Marouf, E. A., and Tyler, G. L.: 1982, Science 217, 243-245.

Marouf, E. A., Tyler, G. L., Zebker, H. A., Simpson, R. A., and Eshleman, V. R: 1983, Icarus 54, $189-211$.

Marouf, E. A., and Tyler, G. L.: 1985, Adv. Space Res. 5, 117-120.

Marouf, E. A., Tyler, G. L., and Rosen, P. A.: 1986, Icarus 68, 120-166.

Marouf, E. A., and Tyler, G. L. 1986, Nature 323, 31-35.

Marouf, E. A., Gresh, D. L., and Tyler, G. L.: 1987, Bull. Am. Astron. Soc. 19, 883.

Marouf, E. A.: 1994, Bull. Am. Astron. Soc. 26, 1150.

Marouf, E. A.: 1996, Bull. Am. Astron. Soc. 28, 1126.

Marouf, E. A.: 1997, Bull. Am. Astron. Soc. 29, 1000.

McElroy, M. B.: 1973, Space Sci. Rev. 14, 460. 
McKay, C. P., Pollack, J. B., and Courtin, R.: 1989, Icarus 80, 23-53.

Melbourne, W. G., Davis, E. S., Duncan, C. B., Hajj, G. A., Hardy, K. R., Kursinski, E. R., et al.: 1994, The Application of Spaceborne GPS to Atmospheric Limb Sounding and Global Change Monitoring, JPL Pub. 94-18, Jet Propulsion Laboratory, California Institute of Technology, Pasadena, CA.

Michel, F. C.: 1982, Astrophys. Lett. 22, 101-102.

Muhleman, D. O., Grossman, A. W., Butler, B. J., and Slade, M. A. 1990, Science 248, 975-980.

Muhleman, D. O., Grossman, A. W., Slade, M. A., and Butler, B. J.: 1992, Bull. Am. Astron. Soc. 24, 954.

Muller, P. M., and Sjogren, W. L.: 1968, Science 161, 680-684.

Nagy, A. F., and Cravens, T. E.: 1998, Planet. Space Sci 46, 1149-1156.

Newell, A. C., and Baird, R. C.: 1965, J. Appl. Phys. 36, 3751-3759.

Newman, M., Schubert, G., Kliore, A. J., and Patel, I. R.: 1984, J. Atmos. Sci. 41, 1901-1913.

Nicholson, P., and Porco, C.: 1988,J. Geophys. Res. 93, 209.

Niemann, H. B., Atreya, S. K., et al.: 1996, Science 272, 848-849.

Phinney, R. A., and Anderson, D. L.: 1968, J. Geophys. Res. 73, 1819.

Plescia, J. B., and Boyce, J. M.: 1982, Nature 295, 285-290.

Porco, C., Nicholson, P. D., Borderies, N., Danielson, G. E., Goldreich, P., Holberg, J. B., et al.: 1984, Icarus 60, 1-16.

Porco, C. C., and Nicholson, P. D.: 1987, Icarus 72, 437-467.

Prentice, A. J. R.: 1980, JPL Publication, 80-80.

Prentice, A. J. R.: 1984, Earth Moon Planets 30, 209-228.

Rappaport, N.: 1994, JPL Interoffice Memorandum 314.6-1549.

Rappaport, N.: 1995, JPL Interoffice Memorandum 312.D-95-105.

Rappaport, N., Bertotti, B., Giampieri, G., and Anderson, J. D.: 1997, Icarus 126, 313-323.

Rappaport, N.: 1998, Icarus 132, 36-42.

Reasenberg, R. D., Shapiro, I. I., MacNeil, P. E., Goldstein, R. B., Breidenthal, J. C., Brenkle, J. P., Cain, D. L., et al.: 1979, Astrophys. J. 234, L219-L221.

Riley, A. L., Antsos, S., Armstrong, J., Kinman, P., Wahlquist, H., Bertotti, B., et al.: 1990, Cassini Ka-band Doppler and Enhanced Telecommunications System Study, Report jointly sponsored by NASA and ASI.

Rosen, P. A.: 1989, Waves in Saturn's Rings Probed by Radio Occultation, Ph.D. Dissertation, Stanford University, Stanford, CA.

Rosen, P. A., and Lissauer, J. J.: 1988, Science 241, 690-694.

Rosen, P. A., Tyler, G. L., and Marouf, E. A.: 1991a, Icarus 93, 3-24.

Rosen, P. A., Tyler, G. L., and Marouf, E. A.: 1991b, Icarus 93, 25-44.

Sagan, C., and Dermott, S. F.: 1982, Nature 300, 731-733.

Samuelson, R. E., Hanel, R. A., Kunde, V. G., and Maguire, W. C.: 1981, Nature 292, 688-693.

Samuelson, R. E.: 1983, Icarus 53, 364-387.

Samuelson, R. E., Nath, N. R., and Borysow, A.: 1997, Planet. Space Sci. 45, 959-980.

Savich, N. A., et al.: 1986, Radiotekhnika i Elektronika 31, 2113.

Schubert, G., Limonadi, D., Anderson, J. D., Campbell, J. K., and Giampieri, G.: 1994, Icarus 111, 433-440.

Schubert, G., Limonadi, D., Anderson, J. D., Campbell, J. K., and Giampieri, G.: 1994, Icarus 111, 433-440.

Schunk, R. W. and Nagy, A. F: 2000, Ionospheres: Physical, Plasma and Chemical Processes, Cambridge University Press, Cambridge.

Showalter, M. R., Cuzzi, J. N., Marouf, E. A., and Esposito, L. W.: 1986, Icarus 66, 297-323.

Shu, F. H., Cuzzi, J. N., and Lissauer, J. J.: 1983, Icarus 53, 185-206.

Shu, F. H.: 1984, in: Greenberg, R. and Brahic, A., (eds.), Planetary Rings, University of Arizona Press, Tucson, pp. 513-561. 
Simpson, R. A., Tyler, G. L, Marouf, E. A., Zebker, H. A., and Eshleman, V. R: 1984, IEEE Trans. Geosci. Remote Sens. 22, 656-665.

Smith, B. A., Soderblom, L., Batson, R., Bridges, P., Inge, J., Masursky, H., et al.: 1982, Science 215, 504-537.

Smith, P. H., Lemmon, M. T., Caldwell, J. J., Allison, M. D., and Sromovsky, L. A.: 1994, Bull. Am. Astron. Soc. 26, 1181.

Sohl, F., Sears, W. D., and Lorenz, R. D.: 1995, Icarus 115, 278-294.

Stevenson, D. J.: 1992, Proceedings of Symposium on 'Titan' (Toulouse, France, 9-12 September 1991), ESA Publication: \#338, pp. 29-33.

Strobel, D. F., Hall, D. T., Zhu, X., and Summers, M. E.: 1993, Icarus 103, 333-336.

Sundstrom, G., et al.: 1994, Science 263, 785-787.

Synnott, S. P., Peters, C. F., Smith, B. A., and Morabito, L. A.: 1981, Science 212, 191-192.

Thorne, K. S.: 1987, Hawking, S., and Israel, W. (eds.), 300 Years of Gravitation, Cambridge University Press, Cambridge, pp.330-458.

Tinto, M. and Armstrong, J. W.: 1991, Ap. J. 372, 545-553.

Tinto, M. and Armstrong, J. W.: 1998, Phys. Rev. D, 58042002.

Tortora, P., Iess, L., and Ekelund, J. E.: 2003, The Cassini multifrequency link performance during 2002 Solar Conjunction Proceedings, IEEE Aerospace Conference, Big Sly, Montana, March $8-15,2003$.

Tortora, P., Iess, L., Bordi, J. J., Ekelund, J. E., and Roth, D.C.: Precise Cassini Navigation during Solar Conjunctions through multifrequency plasma calibrations, J. Guidance, Control and Dyr. 27, 251-257.

Tyler, G. L., Eshleman, V. R., Anderson, J. D., Levy, G. S., Lindal, G. F., Wood, G. E., et al.: 1981, Science 212, 201-206.

Tyler, G. L., Eshleman, V. R., Anderson, J. D., Levy, G. S., Lindal, G. F., Wood, G. E., et al.: 1982, Science 215, 553-558.

Tyler, G. L., Marouf, E. A., Simpson, R. A., Zebker, H. A., and Eshleman, V. R.: 1983, Icarus 54, $160-188$.

Tyler, G. L., Sweetnam, D. N., Anderson, J. D., Campbell, J. K., Eshleman, V. R., Hinson, D. P., et al.: 1986, Science 233, 79-84.

Tyler, G. L.: 1987, Proc. IEEE 75, 1404-1431.

Tyler, G. L., Sweetnam, D. N., Anderson, J. D., E. Borutzki, Campbell, J. K., Kursinski, E. R., et al.: 1989, Science 246, 1466-1473.

Vervack, Jr, R. J., and Sandel, B. R.: 1998, B.A.A.S, 30.

Vervack, Jr, R. J., Sandel, B. R., and Strobel, D. F.: 2004, Icarus 170, 91-112.

Wahlquist, H. D., Anderson, J. D., Estabrook, F. B., and Thorne, K. S.: 1977, Atti dei Convegni Lincei 34, 335-350.

Wahlquist, H. D.: 1987, Gen. Rel. Grav. 19, 1101-1113.

Waite J. A., Jr., and Cravens, T. E.: 1987, Adv. Space Res. 7, 119.

Ward, W. R.: 1981, Geophys. Res. Lett. 8, 641-643.

Will, C. M.: 1993, Theory and Experiment in Gravitational Physics, Cambridge University Press, Cambridge.

Woo, R., and Habbal, S. R.: 1997, Geophys. Res. Lett. 24, 1159-1162.

Yakovlev, O. I., Matyugov, S. S., and Gubenko, V. N.: 1991, Icarus 94, 493-510.

Yelle, R. V: 1991, Astrophys. J. 383, 380-400.

Yung, Y. L., Allen, M., and Pinto, J. P.: 1984, Ap. J. Supp. 55, 465-506.

von Zahn, U., and Hunten, D. M.: 1996, Science 272, 849-851.

Zahnle, K., Pollack, J. B., D. Grinsppon, and Dones, L.: 1992, Icarus 95, 1-23.

Zebker, H. A., Tyler, G. L., and Marouf, E. A.: 1983, Icarus 56, 209-228.

Zebker, H. A., and Tyler, G. L.: 1984, Science 223, 396-398.

Zebker, H. A., Marouf, E. A., and Tyler, G. L.: 1985, Icarus 64, 531-548. 